\title{
Gradient Estimates for Solutions to Divergence Form Elliptic Equations with Discontinuous Coefficients
}

\author{
Yan Yan Li \& Michael Vogelius
}

\author{
Communicated by R. V. KoHN
}

\begin{abstract}
In this paper we derive global $W^{1, \infty}$ and piecewise $C^{1, \alpha}$ estimates for solutions to divergence form elliptic equations with piecewise Hölder continuous coefficients. The novelty of these estimates is that, even though they depend on the shape and on the size of the surfaces of discontinuity of the coefficients, they are independent of the distance between these surfaces.
\end{abstract}

\section{Introduction}

The purpose of this paper is to establish gradient estimates for solutions to a class of divergence form elliptic equations with discontinuous coefficients. Our work is stimulated by the study of composite media with closely spaced interfacial boundaries. A composite medium would for this purpose be represented by a bounded domain $D$, divided into a finite number of subdomains. The physical characteristics of the medium are smooth (e.g., constant) in each subdomain, but they have discontinuities across the surfaces separating the subdomains. A simple, two dimensional example, which very well illustrates the main feature of our estimates, would have the domain $D \subset \mathbb{R}^{2}$ model the cross-section of a fiber-reinforced composite. All the subdomains except one are simply connected and represent the cross-sections of the fibers (typically these will be disks or ellipsoids, depending on whether the cross-section is perpendicular to a particular fiber or not); the remaining subdomain represent the matrix surrounding the fibers. We suppose the shear modulus of the fibers is a constant $\left(0<a_{0}<\infty\right)$, different from the constant shear modulus of the matrix (=1, say). Using a standard model of anti-plane shear we then get the equation

$$
\partial_{i}\left(a(x) \partial_{i} u\right)=0 \quad \text { in } D,
$$


with appropriate (e.g., Dirichlet) boundary conditions on $\partial D$. The function $u$ represents the out of plane elastic displacement, and the coefficient $a(x)$ is given by

$$
\begin{array}{ll}
a(x)=a_{0} & \text { for } x \text { inside the subdomains representing the fibers, } \\
a(x)=1 & \text { elsewhere in } D .
\end{array}
$$

The most important quantities from an engineering point of view are the stresses, in this case represented by the gradient of $u$. A question of particular interest - and indeed the question with which Ivo Babuska initially piqued our interest in this problem area - is the question of whether the stresses remain uniformly bounded, even when fibers touch or nearly touch. It has been shown in various papers that when $a_{0}$ is 0 or $\infty$ the stresses generally become unbounded as fibers get close [4], [9]. For finite and strictly positive $a_{0}$, it has been shown in [3] that the stresses remain bounded for circular touching fibers of comparable radii. This special result was achieved using a Möbius transformation and maximum principles. To the best of our knowledge it has until now not been established any generality whether the stresses remain bounded or "blow up", when fibers touch or nearly touch, and $0<a_{0}<\infty$. In this paper we formulate and establish a general result concerning the structure of solutions to a large class of divergence form elliptic equations with discontinuous coefficients, which in particular may be applied to give a definitive answer to the above question. Our result establishes a uniform bound on $\nabla u$; this bound depends on $a_{0}$ and on the size and shape of the fibers (more specifically on the $C^{1, \alpha}$ modulus of the total boundary of all the subdomains). The bound is independent of the fiber locations and thus it is in particular independent of the distance between the fibers (which may even vanish).

We now proceed to state the main results of this paper. To do so we need to make our notation and assumptions more precise. Let $D$ be a bounded domain in $\mathbb{R}^{n}$ with a $C^{1, \alpha}$ boundary, $0<\alpha<1$, and let $D_{m}, 1 \leqq m \leqq L$, be a finite number of disjoint subdomains of $D$, each with a $C^{1, \alpha}$ boundary. Furthermore suppose that $\bar{D}=\cup_{m=1}^{L} \bar{D}_{m}$. Given $\bar{x} \in \bar{D}$, let $B_{r}(\bar{x})$ denote the ball of radius $r$, centered at $\bar{x}$. We reset $\bar{x}$ to be the origin of our coordinate system. We suppose there exist $r>0$ and an appropriate rotation of our fixed coordinate system, such that the set $\left(\cup_{m=1}^{L} \partial D_{m}\right) \cap B_{r}(\bar{x})$ consists of the graphs of a finite number of $C^{1, \alpha}$ functions (of $n-1$ variables). Let $l(\bar{x}, r)$ denote the number of these functions, and let $K(\bar{x}, r)$ denote the maximum of their $C^{1, \alpha}$ norms. The number

$$
\mathcal{K}=\sup _{\bar{x} \in \bar{D}} \inf _{r>0}\left\{K(\bar{x}, r)+l(\bar{x}, r)+\frac{1}{r}\right\}
$$

is referred to as the $C^{1, \alpha}$ modulus of the total boundary set $\cup_{m=1}^{L} \partial D_{m}$. One immediately sees that the total number of subdomains, $L$, is bounded by a constant that depends on $\mathcal{K}, n$ and $D$.

To consider a simple, but important example, suppose all but one of the domains $D_{m}$ are convex (and $C^{2}$ ). Then, given any $\bar{x}$ in $D$, there exists $r>0$ such that $\left(\cup_{m=1}^{L} \partial D_{m}\right) \cap B_{r}(\bar{x})$ consists of the graphs of at most two functions. The $C^{1, \alpha}$ modulus of the total boundary set $\cup_{m=1}^{L} \partial D_{m}$ is bounded by a constant that only depends on the dimension, $n$, and the maximal curvature of the surfaces $\partial D_{m}$, but 
is independent of their mutual distances. It is clear that $\mathcal{K}$ becomes unbounded as the diameter of one (or more) of the domains $D_{m}$ approaches 0 .

Let $A^{(m)} \in C^{\mu}\left(\bar{D}_{m}\right)(0<\mu<1)$ be a symmetric, positive definite matrixvalued function, and define

$$
A(x)=A^{(m)}(x), \quad x \in D_{m}, 1 \leqq m \leqq L .
$$

Let $0<\bar{\lambda}<\bar{\Lambda}<\infty$ denote ellipticity constants associated with $A$, i.e.,

$$
\bar{\lambda} \leqq A(x) \leqq \bar{\Lambda}
$$

Similarly, let $g^{(m)} \in C^{\mu}\left(\bar{D}_{m}, \mathbb{R}^{n}\right)$, and define

$$
g(x)=g^{(m)}(x), \quad x \in D_{m}, 1 \leqq m \leqq L .
$$

Finally, suppose

$$
h \in L^{\infty}(D)
$$

and

$$
\varphi \in C^{1, \mu}(\partial D) \text {. }
$$

The first of our results concerns $C^{1, \alpha^{\prime}}$ interior estimates. In brief, this result asserts that the restriction of the solution $u$ to each subdomain $D_{m}$ may be extended (to $D_{\varepsilon}=\{x \in D \mid \operatorname{dist}(x, \partial D)>\varepsilon\}$ ) as a $C^{1, \alpha^{\prime}}$ function, with a norm that is independent of the distances between the subdomain interfaces.

Theorem 1.1. Let A, g, and h satisfy (1)-(4). Suppose $\alpha^{\prime}$ satisfies at the same time $0<\alpha^{\prime} \leqq \mu$ and $\alpha^{\prime}<\frac{\alpha}{(\alpha+1) n}$, and supose $\varepsilon>0$. There exists a constant $C$ depending only on $D, n, \alpha, \alpha^{\prime}, \varepsilon, \bar{\lambda}, \bar{\Lambda},\left\|A^{(m)}\right\|_{C^{\alpha^{\prime}}\left(\bar{D}_{m}\right)}$ and the $C^{1, \alpha}$ modulus of $\cup_{m=1}^{L} \partial D_{m}$, such that if $u \in H^{1}(D)$ is a solution to

$$
\partial_{i}\left(A_{i j} \partial_{j} u\right)=h+\partial_{i} g_{i}, \quad \text { in } D,
$$

then

$$
\max _{1 \leqq m \leqq L}\|u\|_{C^{1, \alpha^{\prime}}\left(\bar{D}_{m} \cap D_{\varepsilon}\right)} \leqq C\left(\|u\|_{L^{\infty}(D)}+\|h\|_{L^{\infty}(D)}+\max _{1 \leqq m \leqq L}\left\|g^{(m)}\right\|_{C^{\alpha^{\prime}\left(\bar{D}_{m}\right)}}\right),
$$

where $D_{\varepsilon}=\{x \in D \mid \operatorname{dist}(x, \partial D)>\varepsilon\}$.

We have a similar result concerning $C^{1, \alpha^{\prime}}$ boundary estimates.

Theorem 1.2. Let $A, g, h$, and $\varphi$ satisfy (1)-(5). Suppose $\alpha^{\prime}$ satisfies at the same time $0<\alpha^{\prime} \leqq \mu$ and $\alpha^{\prime}<\frac{\alpha}{(\alpha+1) n}$, and suppose $r>0$. There exists a constant $C$ depending only on $n, \alpha, \alpha^{\prime}, r, \bar{\lambda}, \bar{\Lambda},\left\|A^{(m)}\right\|_{C^{\alpha^{\prime}}\left(\bar{D}_{m}\right)}$ and the $C^{1, \alpha}$ modulus of $\cup_{m=1}^{L} \partial D_{m}$, such that if, for some $\bar{x} \in \partial D, u \in H^{1}\left(D \cap B_{2 r}(\bar{x})\right)$ is a solution to

$$
\begin{aligned}
\partial_{i}\left(A_{i j} \partial_{j} u\right) & =h+\partial_{i} g_{i}, & & \text { in } D \cap B_{2 r}(\bar{x}), \\
u & =\varphi, & & \text { on } \partial D \cap B_{2 r}(\bar{x}),
\end{aligned}
$$


then

$$
\begin{aligned}
\max _{1 \leqq m \leqq L}\|u\|_{C^{1, \alpha^{\prime}}\left(\bar{D}_{m} \cap B_{r}(\bar{x})\right)} \leqq C\left(\|u\|_{L^{\infty}\left(D \cap B_{2 r}(\bar{x})\right)}+\|\varphi\|_{C^{1, \alpha^{\prime}}\left(\partial D \cap B_{2 r}(\bar{x})\right)}\right. \\
\left.+\|h\|_{L^{\infty}\left(D \cap B_{2 r}(\bar{x})\right)}+\max _{1 \leqq m \leqq L}\left\|g^{(m)}\right\|_{C^{\alpha^{\prime}}\left(\bar{D}_{m} \cap B_{2 r}(\bar{x})\right)}\right) .
\end{aligned}
$$

We note that in the past two theorems the norm $\|u\|_{L^{\infty}}$ on the right-hand side can be replaced by the norm $\|u\|_{L^{2}}$, simply by evoking the local $L^{\infty}$ estimates of De Giorgi and Nash. Combining the above interior and boundary estimates with the maximum principle (see for instance [8] Theorem 8.16) we arrive at the following $C^{1, \alpha^{\prime}}$ global estimate. In brief, this estimate asserts that the restriction of the solution $u$ to each subdomain $D_{m}$ can be extended as a $C^{1, \alpha^{\prime}}$ function, with a norm that is bounded independently of the distances between the subdomain interfaces.

Corollary 1.3. Let $A, g, h$, and $\varphi$ satisfy (1)-(5) and suppose $0<\alpha^{\prime} \leqq \mu$ and at the same time $\alpha^{\prime}<\frac{\alpha}{(\alpha+1) n}$. There exists a constant $C$ depending only on $D, n, \alpha, \alpha^{\prime}, \bar{\lambda}, \bar{\Lambda},\left\|A^{(m)}\right\|_{C^{\alpha^{\prime}}\left(\bar{D}_{m}\right)}$ and the $C^{1, \alpha}$ modulus of $\cup_{m=1}^{L} \partial D_{m}$, such that if $u \in H^{1}(D)$ is a solution to

$$
\begin{aligned}
\partial_{i}\left(A_{i j} \partial_{j} u\right) & =h+\partial_{i} g_{i}, & & \text { in } D, \\
u & =\varphi, & & \text { on } \partial D,
\end{aligned}
$$

then

$$
\max _{1 \leqq m \leqq L}\|u\|_{C^{1, \alpha^{\prime}}\left(\bar{D}_{m}\right)} \leqq C\left(\|\varphi\|_{C^{1, \alpha^{\prime}}(\partial D)}+\|h\|_{L^{\infty}(D)}+\max _{1 \leqq m \leqq L}\left\|g^{(m)}\right\|_{C^{\alpha^{\prime}\left(\bar{D}_{m}\right)}}\right) .
$$

Through our assumptions about $\partial D_{m}, 1 \leqq m \leqq L$, we have excluded the possibility that the surfaces of discontinuity touch. This is not essential and, by means of a limiting argument, our theorems easily carry over to "touching" surfaces as well. We briefly comment on this at the beginning of Section 4.

When $\varphi$ belongs to $C^{v}(\partial \Omega)$ instead of $C^{1, \mu}(\partial \Omega)$, we may establish boundary and global $C^{v}$ estimates for solutions. Such estimates do not automatically follow from the De Giorgi-Nash estimates, which give Hölder regularity for some Hölder exponent depending on the ellipticity constants $\bar{\lambda}$ and $\bar{\Lambda}$. It is essential for these estimates that the boundaries $\partial D_{m}$ be $C^{1, \alpha}$, and that the coefficients $A^{(m)}$ be $C^{\mu}$. We refer the reader to Section 7 for the exact statement of these results.

At this point our results are limited to scalar equations, even though numerical evidence suggests that similar bounds hold for certain elliptic system, e.g., the equations of elasticity [2]. There are several other interesting open problems, such as for instance, (a): Does the constant $C$ in our estimates really have to depend on $l(x, r)$, the local number of curves of discontinuity? (currently it does, through the $C^{1, \alpha}$ modulus $\mathcal{K}$ ) or (b): How does $C$ depend on the ellipticity constants $\bar{\lambda}$ and $\bar{\Lambda}$ ? But the most interesting open question is probably, (c): Do similar estimates hold for higher order norms of the solution, assuming of course all the data are appropriately smooth? 
Concerning question (a) we feel the answer most likely is that the constant $C$ is independent of $l(x, r)$. Among other things we base this on the fact that the constants in two important steps of the proof (Proposition 2.1 and Proposition 3.2) are $l$-independent. Concerning question (b), the results in [4] and [9], mentioned earlier, imply that $C$ has to "blow up" as the ellipticity constants degenerate. We feel there is some hope that a refinement of the analysis presented here may give some more specific information about the behaviour of $C$.

We indirectly address question (c) in a very special two-dimensional situation (and with $g=h=0$ ) in Section 8. There we show, by the use of quite explicit expansion formulas, that the solution corresponding to two touching circular inhomogeneities (with constant material parameter, $0<a_{0}<\infty$ ) is indeed $C^{\infty}$ on the closure of each of the (three) subdomains. This in itself does not prove that the $C^{\infty}$ norms of the solutions will remain bounded independently of the distance between two nearly touching circular inhomogeneities - but (for $0<a_{0}<\infty$ ) we feel it does give some indication that this may very well be true.

We now describe our methods of proof. In order to do so clearly and briefly, we restrict attention to Theorem 1.1 in the case $h=0$ and $g=0$. Let $\mathcal{A}(\bar{\lambda}, \bar{\Lambda})$ denote the set of measurable, symmetric, positive definite matrix functions $A(x)$ satisfying

$$
\bar{\lambda} \leqq A(x) \leqq \bar{\Lambda}
$$

We define a scaling invariant subclass of $\mathcal{A}(\bar{\lambda}, \bar{\Lambda})$, denoted $\overline{\mathcal{A}}(\bar{\lambda}, \bar{\Lambda})$, as follows. Let $L_{1}, \cdots, L_{l}$ be any $l$ parallel hyperplanes in $\mathbb{R}^{n}$ which divide $\mathbb{R}^{n}$ into $l+1$ regions, denoted as $R_{1}, \cdots, R_{l+1}$. Let $\bar{A}^{(1)}, \cdots, \bar{A}^{(l+1)}$ be any $l+1$ symmetric, positive definite constant matrices in $\mathcal{A}(\bar{\lambda}, \bar{\Lambda})$ and define

$$
\bar{A}_{i j}(x)=\bar{A}_{i j}^{(m)}, \quad x \in R_{m}, 1 \leqq m \leqq l+1 .
$$

The subclass $\overline{\mathcal{A}}(\bar{\lambda}, \bar{\Lambda})$ consists of all such matrix functions $\bar{A}$.

The classical Schauder estimates, Cordes-Nirenberg estimates, and $W^{2, p}$ estimates can be viewed as perturbation theories from the corresponding estimates for solutions to the Laplace equation. The approach here is to treat our equations as perturbations to the following basic equations

$$
\partial_{i}\left(\bar{A}_{i j}(x) \partial_{j}\right) v=0
$$

with $\bar{A} \in \overline{\mathcal{A}}(\bar{\lambda}, \bar{\Lambda})$.

To establish Theorem 1.1 in the case $h=0$ and $g=0$, we thus first study elliptic regularity estimates for solutions to (6), the main point being that the hyperplanes in the definition of $\overline{\mathcal{A}}(\bar{\lambda}, \bar{\Lambda})$ are allowed to get arbitrarily close to each other, while we still obtain estimates that are uniform in $\bar{A} \in \overline{\mathcal{A}}(\bar{\lambda}, \bar{\Lambda})$. In Proposition 2.1 we establish, with the help of the Cacciopolli inequality and the De Giorgi-Nash estimates, (interior) estimates for all derivatives of the solution $v$ in each region $\bar{R}_{m}$. More precisely, with $\Omega=(-1,1)^{n}$, we show that for any positive integer $k$, any $\varepsilon>0$, any $\bar{A} \in \overline{\mathcal{A}}(\bar{\lambda}, \bar{\Lambda})$, and any solution $v$ to (6)

$$
\max _{1 \leqq m \leqq l+1}\|v\|_{C^{k}\left(\bar{R}_{m} \cap(1-\varepsilon) \Omega\right)} \leqq C\|v\|_{L^{\infty}(\Omega)} .
$$


The constant $C$ depends only on $n, \varepsilon, k, \bar{\lambda}$, and $\bar{\Lambda}$.

Starting from (7) we use perturbation methods, inspired by the work of Caffarelli (see [6] and [5]) to show that for any $q>n, 0<\bar{\alpha}<1$, there exists some positive number $\varepsilon_{0}$, depending only on $n, q, \bar{\alpha}, \bar{\lambda}$ and $\bar{\Lambda}$, such that if $A \in \mathcal{A}(\bar{\lambda}, \bar{\Lambda})$ and $\bar{A} \in \overline{\mathcal{A}}(\bar{\lambda}, \bar{\Lambda})$ satisfy

$$
\sup _{0<r<1} r^{-\bar{\alpha}}\left(f_{r \Omega}|A(x)-\bar{A}(x)|^{q}\right)^{1 / q} \leqq \varepsilon_{0},
$$

and $u \in H^{1}(\Omega)$ is a solution to

$$
\partial_{i}\left(A_{i j}(x) \partial_{j}\right) u=0 \quad \text { in } \Omega,
$$

with

$$
\|u\|_{L^{\infty}(\Omega)} \leqq 1
$$

then there exists $p(x)$, a continuous, piecewise linear solution to

$$
\partial_{i}\left(\bar{A}_{i j}(x) \partial_{j}\right) p(x)=0 \quad \text { in } \frac{1}{4} \Omega,
$$

whose coefficients are bounded in absolute value by $C$, and that satisfies

$$
|u(x)-p(x)| \leqq C|x|^{1+\bar{\alpha}}, \quad x \in \frac{1}{4} \Omega .
$$

The constant $C$ depends only on $n, q, \bar{\alpha}, \bar{\lambda}$, and $\bar{\Lambda}$.

We then show that, under the hypotheses of Theorem 1.1, the condition (8) may be verified at every point $\bar{x}$ in $D$, through a harmless translation and dilatation, and by appropriate selection of $\bar{A}_{\bar{x}} \in \mathcal{A}(\bar{\lambda}, \bar{\Lambda})$. The $L^{\infty}$ interior estimates for the gradient of solutions to the equation $\partial_{i}\left(A_{i j} \partial_{j} u\right)=0$ follow immediately. The Hölder interior estimates for the gradient require some further work, since at different points $\bar{x} \in \Omega$, the orientation of the hyperplanes associated with the matrices $\bar{A}_{\bar{x}}(x)$ differ by a rotation, determined by the geometry of $D_{m}$, and since $p_{\bar{x}}(x)$ is only piecewise linear given a fixed set of planes. To deal with these local changes in orientation, and to obtain Hölder estimates for the gradients in $D_{m}$, we need to study the relations to gradients in sets, $D_{j}$, with indices $j \neq m$.

The organization of the paper is as follows. In Section 2 we give a proof of a generalized version of the estimate (7); we refer to this as our basic proposition since it lies at the foundation of our later perturbation arguments. In Section 3 we establish our main perturbation result; based on the assumption (8), the scaling invariance of our equation and the scaling invariance of the class $\overline{\mathcal{A}}(\bar{\lambda}, \bar{\Lambda})$, this result permits us to construct the required piecewise linear approximation. Instead of proceeding immediately to a proof of Theorem 1.1, we give in Section 4 a proof of a simplified version. This simplified version is of interest in itself, but more importantly its proof clearly illustrates the main arguments necessary for the verification of (8), and it also clearly illustrates how the existence of $p$ may be used to derive a uniform gradient estimate. In Section 5 we introduce the additional ingredients that are required for a full proof of Theorem 1.1. Section 6 is devoted to an outline of the proof of the boundary estimates, i.e., Theorem 1.2. In Section 7 we present the result on global Hölder regularity, briefly mentioned earlier. In Section 8 we address an aspect 
of the high order regularity question (c) formulated earlier. We establish the $C^{\infty}$ piecewise smoothness of solutions corresponding to a two-dimensional medium with touching circular inhomogeneities. There is an appendix to this paper, which is devoted to a proof of the piecewise smoothness of solutions to a problem with a single, smooth interface and natural transmission boundary conditions. This fairly classical result is needed in order to establish our basic proposition. We provide a proof, suggested by L. Nirenberg, for the convenience of the reader (to make the paper more self-contained).

\section{Basic proposition}

Let $\Omega$ denote the $n$-dimensional cube

$$
\Omega=\left\{x=\left(x_{1}, \cdots, x_{n}\right):\left|x_{i}\right|<1\right\},
$$

and suppose the constants $c_{m}, 0 \leqq m \leqq l+1$, satisfy

$$
-1 \equiv c_{0}<c_{1}<\cdots<c_{l+1} \equiv 1 .
$$

We define "strips", $\Omega_{m}$, as follows:

$$
\Omega_{m}=\left\{x \in \Omega \mid c_{m-1}<x_{n}<c_{m}\right\}, \quad 1 \leqq m \leqq l+1 .
$$

Let $\left\{\bar{A}^{(m)}\right\}_{m=1}^{l+1}=\left\{\left(\bar{A}_{i j}^{(m)}\right)\right\}_{m=1}^{l+1}$ be a set of symmetric, positive definite matrices, with $0<\bar{\lambda}<\bar{A}^{(m)}<\bar{\Lambda}<\infty$, and define the matrix function $\bar{A}(x)$ by

$$
\bar{A}_{i j}(x)=\bar{A}_{i j}^{(m)}, \quad x \in \Omega_{m}, \quad 1 \leqq m \leqq l+1 .
$$

The function $\bar{A}$ is in $\overline{\mathcal{A}}(\bar{\lambda}, \bar{\Lambda})$. Similarly, let $\left\{\bar{G}^{(m)}\right\}_{m=1}^{l+1}$ be a set of $n$-vectors, and define

$$
\bar{G}(x)=\bar{G}^{(m)}, \quad x \in \Omega_{m}, \quad 1 \leqq m \leqq l+1 .
$$

Finally suppose $\left\{\bar{H}^{(m)}\right\}_{m=1}^{l+1}$ is a set of constants, and define

$$
\bar{H}(x)=\bar{H}^{(m)}, \quad x \in \Omega_{m}, \quad 1 \leqq m \leqq l+1 .
$$

The following proposition plays a fundamental role in our proofs of Theorem 1.1 and Theorem 1.2.

Proposition 2.1 (Basic Proposition). Let $\bar{A}, \bar{G}$ and $\bar{H}$ be as above. Suppose $k$ is a non-negative integer and suppose $\varepsilon>0$. There exists a constant $C=C(\varepsilon, k, n$, $\bar{\lambda}, \bar{\Lambda})$, such that if $v \in H^{1}(\Omega)$ is a solution to

$$
\partial_{i}\left(\bar{A}_{i j}(x) \partial_{j}\right) v=\bar{H}+\partial_{i} \bar{G}_{i} \quad \text { in } \Omega,
$$

then

$$
\max _{1 \leqq m \leqq l+1}\|v\|_{C^{k}\left(\bar{\Omega}_{m} \cap(1-\varepsilon) \Omega\right)} \leqq C\left(\|v\|_{L^{\infty}(\Omega)}+\|\bar{H}\|_{L^{\infty}(\Omega)}+\|\bar{G}\|_{L^{\infty}(\Omega)}\right) .
$$


Remark 2.1. It is not difficult to see that we only need to establish Proposition 2.1 for some $\bar{\varepsilon}=\bar{\varepsilon}(k, n, \bar{\lambda}, \bar{\Lambda})>0$, the reason being that for any $\bar{x} \in(1-\varepsilon) \Omega$, we may apply Proposition 2.1 with $\bar{\varepsilon}$ to the function $\bar{v}(x)=v(\bar{x}+\varepsilon x)$.

Remark 2.2. The fact that $v$ is in $C^{k}\left(\bar{\Omega}_{m} \cap \Omega\right)$ follows from Theorem 9.1 in the appendix. The aim here is to verify the estimate (10) with a constant $C$ that only depends on $\varepsilon, k, n, \bar{\lambda}$ and $\bar{\Lambda}$, but not on $c_{1}, \ldots, c_{l}$ or $l$.

Proposition 2.1 is a relatively simple consequence of the following four lemmas.

Lemma 2.2. Suppose the hypotheses are as in Proposition 2.1. Let $D_{x^{\prime}}^{k}$ denote any derivative of order less than or equal to $k$ with respect to the $n-1$ variables $x^{\prime}=\left(x_{1}, \cdots, x_{n-1}\right)$. Given any $v \in H^{1}(\Omega)$ satisfying (9) the function $D_{x^{\prime}}^{k} v$ remains in $H^{1}(\Omega)$. If the order of $D_{x^{\prime}}^{k}$ is strictly positive then $D_{x^{\prime}}^{k} v$ satisfies the homogeneous version of (9), i.e.,

$$
\partial_{i}\left(\bar{A}_{i j}(x) \partial_{j}\right)\left(D_{x^{\prime}}^{k} v\right)=0 \quad \text { in } \Omega,
$$

and there exists a constant $C=C(\varepsilon, k, n, \bar{\lambda}, \bar{\Lambda})$ such that

$$
\left\|D_{x^{\prime}}^{k} v\right\|_{L^{\infty}((1-\varepsilon) \Omega)} \leqq C\left(\|v\|_{L^{\infty}(\Omega)}+\|\bar{H}\|_{L^{\infty}(\Omega)}+\|\bar{G}\|_{L^{\infty}(\Omega)}\right) .
$$

Proof. In view of (9) it follows from the Cacciopolli inequality (see, for example, [7]) that

$\left\|\partial_{\beta} v\right\|_{L^{2}((1-\varepsilon / 2) \Omega)} \leqq C\left(\|v\|_{L^{\infty}(\Omega)}+\|\bar{H}\|_{L^{\infty}(\Omega)}+\|\bar{G}\|_{L^{\infty}(\Omega)}\right), \quad 1 \leqq \beta \leqq n-1$, with $C=C(\varepsilon, n, \bar{\lambda}, \bar{\Lambda})$. From Theorem 9.1 in the appendix we have that $\partial_{\beta} v \in$ $H^{1}((1-\varepsilon / 2) \Omega)$. We easily see that

$$
\partial_{i}\left(\bar{A}_{i j}(x) \partial_{j}\right)\left(\partial_{\beta} v\right)=0 \quad \text { in }(1-\varepsilon / 2) \Omega
$$

for any $1 \leqq \beta \leqq n-1$. Applying the local De Giorgi-Nash estimates (see, for example, [8]) to $\partial_{\beta} v$, we now get

$$
\begin{aligned}
\left\|\partial_{\beta} v\right\|_{L^{\infty}((1-\varepsilon) \Omega)} & \leqq C\left\|\partial_{\beta} v\right\|_{L^{2}((1-\varepsilon / 2) \Omega)} \\
& \leqq C\left(\|v\|_{L^{\infty}(\Omega)}+\|\bar{H}\|_{L^{\infty}(\Omega)}+\|\bar{G}\|_{L^{\infty}(\Omega)}\right), \quad 1 \leqq \beta \leqq n-1 .
\end{aligned}
$$

We have thus established Lemma 2.2 in the case $k=1$. The general case follows easily by induction since $\partial_{\beta} v, 1 \leqq \beta \leqq n-1$, satisfies the the same type of equation as $v$, only with homogeneous data and with $\Omega$ replaced by $(1-\varepsilon) \Omega$.

We introduce the notation

$$
v^{(m)}=\left.v\right|_{\Omega_{m}} .
$$

The function $v^{(m)}$ is in $C^{\infty}\left(\bar{\Omega}_{m} \cap \Omega\right)$ for any $1 \leqq m \leqq l+1$. In view of (9) (and the regularity result in the appendix) we have

$$
\sum_{i=1}^{n} \bar{A}_{i n}^{(m)} \partial_{i} v^{(m)}-\bar{G}_{n}^{(m)}=\sum_{i=1}^{n} \bar{A}_{i n}^{(m-1)} \partial_{i} v^{(m-1)}-\bar{G}_{n}^{(m-1)}
$$


on $\left\{x_{n}=c_{m-1}\right\} \cap \Omega$, for any $2 \leqq m \leqq l+1$. In other words, the function

$$
\sum_{i=1}^{n} \bar{A}_{i n} \partial_{i} v-\bar{G}_{n}
$$

is globally continuous in $\Omega$. The following first order differential operators are relevant

$$
X^{(m)}=\sum_{i=1}^{n} \bar{A}_{i n}^{(m)} \partial_{i} \quad \text { in } \Omega_{m} .
$$

Lemma 2.3. Suppose the hypotheses are as in Proposition 2.1. There exists a constant $C=C(n, \varepsilon, \bar{\lambda}, \bar{\Lambda})$, such that if $v \in H^{1}(\Omega)$ is a solution to (9) then

$$
\begin{gathered}
\left|X^{(m)} X^{(m)} v^{(m)}(x)\right| \leqq C\left(\|v\|_{L^{\infty}(\Omega)}+\|\bar{H}\|_{L^{\infty}(\Omega)}+\|\bar{G}\|_{L^{\infty}(\Omega)}\right), \\
x \in \Omega_{m} \cap(1-\varepsilon) \Omega
\end{gathered}
$$

for any $1 \leqq m \leqq l+1$.

Proof. A simple calculation yields

$$
\begin{aligned}
X^{(m)}\left(X^{(m)} v^{(m)}\right)= & \bar{A}_{n n}^{(m)} \partial_{i}\left(\bar{A}_{i j}(x) \partial_{j}\right) v^{(m)} \\
& +\sum_{1 \leqq \alpha, \beta \leqq n-1}\left(\bar{A}_{\alpha n}^{(m)} \bar{A}_{\beta n}^{(m)}-\bar{A}_{n n}^{(m)} \bar{A}_{\alpha \beta}^{(m)}\right) \partial_{\alpha \beta} v^{(m)} .
\end{aligned}
$$

Since $\partial_{i}\left(\bar{A}_{i j}(x) \partial_{j}\right) v^{(m)}=\bar{H}^{(m)}+\partial_{i} \bar{G}_{i}^{(m)}\left(=\bar{H}^{(m)}\right)$ in $\Omega_{m}$, the lemma follows from the above identity and Lemma 2.2.

The vector fields $X^{(m)}=\left(\bar{A}_{1 n}^{(m)}, \ldots, \bar{A}_{n n}^{(m)}\right)$ (that give rise to the differential operators of the same name) have the following properties

$$
\frac{X^{(m)} \cdot e_{n}}{\left|X^{(m)}\right|} \geqq \hat{c}>0, \quad \hat{c} \leqq\left|X^{(m)}\right| \leqq 1 / \hat{c}, \quad \forall 1 \leqq m \leqq l+1,
$$

where $e_{n}=(0, \cdots, 0,1)$ and $\hat{c}$ is some constant depending only on $\bar{\lambda}, \bar{\Lambda}$, and $n$. Because of (11), there exist two positive constants $E<\frac{1}{2}$ and $\hat{\varepsilon}_{0}$, depending only on $n, \bar{\lambda}$, and $\bar{\Lambda}$, such that for every $\hat{x} \in \hat{\varepsilon}_{0} \Omega$, the integral curve of the vector fields $\left\{X^{(m)}\right\}$, starting from $\hat{x}$, intersects $x_{n}= \pm E$ before leaving $\frac{1}{2} \Omega$. Here and in the following, an integral curve $\gamma(t)$ of the vector fields $\left\{X^{(m)}\right\}$ means a continuous solution to

$$
\gamma^{\prime}(t)=X^{(m)} \quad \text { whenever } \gamma(t) \in \Omega_{m}, 1 \leqq m \leqq l+1 .
$$

In fact, $\gamma(t)$ is piecewise linear since $X^{(m)}$ is a constant vector field in $\Omega_{m}$. Without loss of generality, we may assume that $-E=c_{l_{0}}$ and $E=c_{l_{1}+1}$ for some $0 \leqq$ $l_{0}<l_{1}+1 \leqq l+1$ (if necessary, we simply add these two constants to the original set $\left.\left\{c_{0}, \cdots, c_{l+1}\right\}\right)$.

We will make use of the following elementary fact. 
Lemma 2.4. Let $\tilde{f}$ be a function on $[0,1]$ with Lipschitz first derivative. Then for some universal constant $C$,

$$
\left\|\tilde{f}^{\prime}\right\|_{L^{\infty}(0,1)} \leqq C\left(\|\tilde{f}\|_{L^{\infty}(0,1)}+\left\|\tilde{f}^{\prime \prime}\right\|_{L^{\infty}(0,1)}\right) .
$$

Lemma 2.5. Suppose the hypotheses are as in Proposition 2.1, and let $\hat{\varepsilon}_{0}$ be as introduced above. There exists a constant $C=C(n, \bar{\lambda}, \bar{\Lambda})$ such that, if $v \in H^{1}(\Omega)$ is a solution to (9), then

$$
\left\|\partial_{n} v\right\|_{L^{\infty}\left(\hat{\varepsilon}_{0} \Omega\right)} \leqq C\left(\|v\|_{L^{\infty}(\Omega)}+\|\bar{H}\|_{L^{\infty}(\Omega)}+\|\bar{G}\|_{L^{\infty}(\Omega)}\right) .
$$

Proof. Let $\gamma$ denote any integral curve of the vector fields $\left\{X^{(m)}\right\}$ that passes through $\hat{\varepsilon}_{0} \Omega$. We know that $\gamma$ intersects $x_{n}= \pm E$ before leaving $\frac{1}{2} \Omega$. Let $t_{0}<t_{1}$ be such that $\gamma\left(t_{0}\right)_{n}=-E$ and $\gamma\left(t_{1}\right)_{n}=E$ (and $\gamma(t) \in \frac{1}{2} \Omega$ for $\left.t_{0} \leqq t \leqq t_{1}\right)$. It is clear that $1 / C \leqq t_{1}-t_{0} \leqq C$ for some constant $C$ depending only on $n, \bar{\lambda}$ and $\bar{\Lambda}$. We define

$$
w(t)=\left(\sum_{i=1}^{n} \bar{A}_{i n} \partial_{i} v-\bar{G}_{n}\right)(\gamma(t)) .
$$

Due to the global continuity of $\sum_{i=1}^{n} \bar{A}_{i n} \partial_{i} v-\bar{G}_{n}$ the function $w$ is clearly Lipschitz on the interval $\left[t_{0}, t_{1}\right]$. It is in fact infinitely often differentiable, except at a finite number of points. The first derivative of $w$ equals $\left(X^{(m)} X^{(m)} v^{(m)}\right)(\gamma(t))$ when $\gamma(t)$ lies in $\Omega_{m}$. Due to Lemma 2.3 we now have

$$
\left\|w^{\prime}\right\|_{L^{\infty}\left(t_{0}, t_{1}\right)} \leqq C\left(\|v\|_{L^{\infty}(\Omega)}+\|\bar{H}\|_{L^{\infty}(\Omega)}+\|\bar{G}\|_{L^{\infty}(\Omega)}\right) .
$$

We also define

$$
f(t)=v(\gamma(t)) \quad \text { and } \quad g(t)=\bar{G}_{n}(\gamma(t)) .
$$

The function $f$ is clearly Lipschitz on the interval $\left[t_{0}, t_{1}\right]$ (and infinitely often differentiable, except at a finite number of points). The function $g$ is uniformly bounded with

$$
\|g\|_{L^{\infty}\left(t_{0}, t_{1}\right)} \leqq\|\bar{G}\|_{L^{\infty}(\Omega)}
$$

The three functions $f, g$ and $w$ are related by $f^{\prime}=w+g$. Set $\tilde{f}(t)=f(t)-$ $\int_{t_{0}}^{t} g(\tau) d \tau$, then $\tilde{f}^{\prime}=w$ and

$$
\left\|\tilde{f}^{\prime \prime}\right\|_{L^{\infty}\left(t_{0}, t_{1}\right)}=\left\|w^{\prime}\right\|_{L^{\infty}\left(t_{0}, t_{1}\right)} \leqq C\left(\|v\|_{L^{\infty}(\Omega)}+\|\bar{H}\|_{L^{\infty}(\Omega)}+\|\bar{G}\|_{L^{\infty}(\Omega)}\right) .
$$

It follows from Lemma 2.4, together with (12) and (13), that

$$
\|w\|_{L^{\infty}\left(t_{0}, t_{1}\right)}=\left\|\tilde{f}^{\prime}\right\|_{L^{\infty}\left(t_{0}, t_{1}\right)} \leqq C\left(\|v\|_{L^{\infty}(\Omega)}+\|\bar{H}\|_{L^{\infty}(\Omega)}+\|\bar{G}\|_{L^{\infty}(\Omega)}\right) .
$$


Due to the definition of $w$ (and the lower bound for $\bar{A}_{n n}$ )

$$
\begin{aligned}
& \left\|\partial_{n} v(\gamma(\cdot))\right\|_{L^{\infty}\left(t_{0}, t_{1}\right)} \\
& \leqq C\left(\|w\|_{L^{\infty}\left(t_{0}, t_{1}\right)}+\sum_{\beta \leqq n-1}\left\|\partial_{\beta} v(\gamma(\cdot))\right\|_{L^{\infty}\left(t_{0}, t_{1}\right)}+\left\|\bar{G}_{n}(\gamma(\cdot))\right\|_{L^{\infty}\left(t_{0}, t_{1}\right)}\right),
\end{aligned}
$$

and since $\gamma$ is an arbitrary integral curve passing through $\hat{\varepsilon}_{0} \Omega$, it now follows, using Lemma 2.2, that

$$
\left\|\partial_{n} v\right\|_{L^{\infty}\left(\hat{\varepsilon}_{0} \Omega\right)} \leqq C\left(\|v\|_{L^{\infty}(\Omega)}+\|\bar{H}\|_{L^{\infty}(\Omega)}+\|\bar{G}\|_{L^{\infty}(\Omega)}\right) .
$$

This completes the proof of Lemma 2.5.

We are now ready for

Proof of Proposition 2.1. Due to Remark 2.1, we only need to establish Proposition 2.1 for some $\bar{\varepsilon}=\bar{\varepsilon}(k, n, \bar{\lambda}, \bar{\Lambda})$. Because of Lemma 2.2, we can apply Lemma 2.5 to $D_{x^{\prime}}^{k+1} v$ (any derivative in the variables $x_{1}, \ldots, x_{n-1}$ of degree $\leqq k+1$ ) to obtain

$$
\begin{aligned}
\left\|D_{x^{\prime}}^{k+1} \partial_{x_{n}} v\right\|_{L^{\infty}\left(\Omega_{m} \cap \hat{\varepsilon}_{1} \Omega\right)} \leqq & C\left(\|v\|_{L^{\infty}(\Omega)}+\|\bar{H}\|_{L^{\infty}(\Omega)}+\|\bar{G}\|_{L^{\infty}(\Omega)}\right), \\
& 1 \leqq m \leqq l+1
\end{aligned}
$$

with $C=C(\varepsilon, k, n, \bar{\lambda}, \bar{\Lambda})$ and $\hat{\varepsilon}_{1}=\hat{\varepsilon}_{1}(n, \bar{\lambda}, \bar{\Lambda})$. Applying $D_{x^{\prime}}^{k}$ to the equation for $v$ on each $\Omega_{m}$, and using Lemma 2.2 and (14), we get

$$
\begin{aligned}
\left\|D_{x^{\prime}}^{k} \partial_{x_{n}}^{2} v\right\|_{L^{\infty}\left(\Omega_{m} \cap \hat{\varepsilon}_{1} \Omega\right)} \leqq & C\left(\|v\|_{L^{\infty}(\Omega)}+\|\bar{H}\|_{L^{\infty}(\Omega)}+\|\bar{G}\|_{L^{\infty}(\Omega)}\right), \\
& 1 \leqq m \leqq l+1 .
\end{aligned}
$$

Applying $D_{x^{\prime}}^{k} \partial_{x_{n}}$ to the equation for $v$ on each $\Omega_{m}$, and using Lemma 2.2 and (14) and (15) (with k replaced by $k+1$ ) we similarly get

$$
\begin{gathered}
\left\|D_{x^{\prime}}^{k} \partial_{x_{n}}^{3} v\right\|_{L^{\infty}\left(\Omega_{m} \cap \hat{\varepsilon}_{1} \Omega\right)} \leqq C\left(\|v\|_{L^{\infty}(\Omega)}+\|\bar{H}\|_{L^{\infty}(\Omega)}+\|\bar{G}\|_{L^{\infty}(\Omega)}\right) \\
1 \leqq m \leqq l+1
\end{gathered}
$$

By induction we obtain a proof of Proposition 2.1 with $\bar{\varepsilon}=\hat{\varepsilon}_{1}$.

It is not primarily Proposition 2.1 that we use later in this paper. Rather we use a corollary concerning the approximation of $v$ by piecewise polynomials. Since our aim is to estimate gradients alone, it suffices to consider approximation by piecewise linear functions. We introduce the following notation

$$
\begin{aligned}
& m_{*}=\min \left\{m: \Omega_{m} \cap \frac{1}{2} \Omega \neq \emptyset\right\}=\min \left\{m:-\frac{1}{2}<c_{m}\right\}, \\
& m^{*}=\max \left\{m: \Omega_{m} \cap \frac{1}{2} \Omega \neq \emptyset\right\}=\max \left\{m: c_{m-1}<\frac{1}{2}\right\} .
\end{aligned}
$$


Corollary 2.6. Let $\bar{A}, \bar{G}$ and $\bar{H}$ be as in Proposition 2.1, and let $v \in H^{1}(\Omega)$ denote a solution to

$$
\partial_{i}\left(\bar{A}_{i j}(x) \partial_{j}\right) v=\bar{H}+\partial_{i}\left(\bar{G}_{i}\right) \quad \text { in } \Omega,
$$

with

$$
\|v\|_{L^{\infty}(\Omega)}+\|\bar{H}\|_{L^{\infty}(\Omega)}+\|\bar{G}\|_{L^{\infty}(\Omega)} \leqq 1 .
$$

There exists a constant $C=C(n, \bar{\lambda}, \bar{\Lambda})$ and a continuous, piecewise linear function

$$
p(x)=a^{(m)}+b^{(m)} \cdot x, \quad x \in \Omega_{m}, 1 \leqq m \leqq l+1,
$$

satisfying

$$
\partial_{i}\left(\bar{A}_{i j}(x) \partial_{j}\right) p(x)=\partial_{i}\left(\bar{G}_{i}\right) \quad \text { in } \Omega \text {, }
$$

such that

$$
|v(x)-p(x)| \leqq C|x|^{2}, \quad x \in \frac{1}{2} \Omega,
$$

and

$$
\left|a^{(m)}\right|+\left|b^{(m)}\right| \leqq C, \quad m_{*} \leqq m \leqq m^{*}
$$

Proof. A continuous, piecewise linear function, $p(x)$, is a solution to (16) if and only if

$$
b^{(m)}=M^{(m)} b^{(m-1)}+\left(0^{\prime}, \frac{1}{\bar{A}_{n n}^{(m)}}\left[\bar{G}_{n}^{(m)}-\bar{G}_{n}^{(m-1)}\right]\right) \quad \text { for all } m,
$$

where $M^{(m)}$ is the $n \times n$ matrix given by

$$
\begin{array}{llrl}
M_{\alpha \beta}^{(m)}=\delta_{\alpha \beta}, & 1 \leqq \alpha, \beta \leqq n-1, \\
M_{\alpha n}^{(m)}=0, & 1 \leqq \alpha \leqq n-1, \\
M_{n \alpha}^{(m)}=\frac{1}{\bar{A}_{n n}^{(m)}}\left[\bar{A}_{\alpha n}^{(m-1)}-\bar{A}_{\alpha n}^{(m)}\right], & 1 \leqq \alpha \leqq n-1,
\end{array}
$$

and

$$
M_{n n}^{(m)}=\frac{\bar{A}_{n n}^{(m-1)}}{\bar{A}_{n n}^{(m)}} .
$$

We also know that $v^{(m)}:=\left.v\right|_{\Omega_{m}}$ and $v^{(m-1)}$ satisfy the same matching conditions at $x_{n}=c_{m-1}$

$$
\nabla v^{(m)}\left(x^{\prime}, c_{m-1}\right)=M^{(m)} \nabla v^{(m-1)}\left(x^{\prime}, c_{m-1}\right)+\left(0^{\prime}, \frac{1}{\bar{A}_{n n}^{(m)}}\left[\bar{G}_{n}^{(m)}-\bar{G}_{n}^{(m-1)}\right]\right) \forall x^{\prime} .
$$


Without loss of generality, suppose

$$
c_{m_{0}-1} \leqq 0<c_{m_{0}} .
$$

By Taylor's expansion and Proposition 2.1,

$$
v^{\left(m_{0}\right)}(x)=v^{\left(m_{0}\right)}(0)+\nabla v^{\left(m_{0}\right)}(0) x+O\left(|x|^{2}\right) \quad x \in \bar{\Omega}_{m_{0}} \cap \frac{1}{2} \Omega,
$$

with $\left|O\left(|x|^{2}\right)\right| \leqq C(n, \bar{\lambda}, \bar{\Lambda})|x|^{2}$. Define

$$
a^{\left(m_{0}\right)}=v^{\left(m_{0}\right)}(0), \quad b^{\left(m_{0}\right)}=\nabla v^{\left(m_{0}\right)}(0),
$$

and define all the remaining $\left\{a^{(m)}\right\},\left\{b^{(m)}\right\}$ by the following relations

$$
\begin{gathered}
b^{(m)}=M^{(m)} b^{(m-1)}+\left(0^{\prime}, \frac{1}{\bar{A}_{n n}^{(m)}}\left[\bar{G}_{n}^{(m)}-\bar{G}_{n}^{(m-1)}\right]\right), \\
a^{(m)}+b^{(m)} \cdot\left(0^{\prime}, c_{m-1}\right)=a^{(m-1)}+b^{(m-1)} \cdot\left(0^{\prime}, c_{m-1}\right) .
\end{gathered}
$$

As previously, $p$ denotes the continuous, piecewise linear function $p(x)=a^{(m)}+$ $b^{(m)} \cdot x, x \in \Omega_{m}$. The function $p$ clearly satisfies (16), since the matching conditions are satisfied by construction. For $m=m_{0}$ the estimate (18) follows immediately from (21) and Proposition 2.1. We now verify this same estimate for $m_{0}<m \leqq m^{*}$. The verification for $m_{*} \leqq m<m_{0}$ proceeds in the same way, but is left to the reader.

We know that $M^{(m)}=\left[N^{(m)}\right]^{-1} N^{(m-1)}$, where $N^{(m)}$ is the $n \times n$ matrix given by

$$
\begin{gathered}
N_{\alpha \beta}^{(m)}=\delta_{\alpha \beta}, \quad 1 \leqq \alpha, \beta \leqq n-1, \\
N_{\alpha n}^{(m)}=0, \quad 1 \leqq \alpha \leqq n-1,
\end{gathered}
$$

and

$$
N_{n j}^{(m)}=\bar{A}_{j n}^{(m)}, \quad 1 \leqq j \leqq n .
$$

The identities (19) and (22) together with Proposition 2.1 imply

$$
\begin{aligned}
& {\left[b^{(m)}-\nabla v^{(m)}\left(0^{\prime}, c_{m-1}\right)\right]} \\
& \quad=\left[N^{(m)}\right]^{-1} N^{(m-1)}\left[b^{(m-1)}-\nabla v^{(m-1)}\left(0^{\prime}, c_{m-2}\right)\right]+O\left(c_{m-1}-c_{m-2}\right)
\end{aligned}
$$

provided $m_{0}+1<m \leqq m^{*}$. By iteration it follows that

$$
\begin{aligned}
& {\left[b^{(m)}-\nabla v^{(m)}\left(0^{\prime}, c_{m-1}\right)\right]} \\
& \quad=\left[N^{(m)}\right]^{-1} N^{\left(m_{0}+1\right)}\left[b^{\left(m_{0}+1\right)}-\nabla v^{\left(m_{0}+1\right)}\left(0^{\prime}, c_{m_{0}}\right)\right]+O\left(c_{m-1}-c_{m_{0}}\right)
\end{aligned}
$$

provided $m_{0}<m \leqq m^{*}$. Here $\left|O\left(c_{m-1}-c_{m_{0}}\right)\right| \leqq C\left(c_{m-1}-c_{m_{0}}\right)$ with a constant $C(n, \bar{\lambda}, \bar{\Lambda})$ that is independent of $l$. We also have

$$
b^{\left(m_{0}+1\right)}-\nabla v^{\left(m_{0}+1\right)}\left(0^{\prime}, c_{m_{0}}\right)=M^{\left(m_{0}+1\right)}\left[b^{\left(m_{0}\right)}-\nabla v^{\left(m_{0}\right)}\left(0^{\prime}, c_{m_{0}}\right)\right]=O\left(c_{m_{0}}\right),
$$


and in combination with (24) this leads to

$$
\left|b^{(m)}-\nabla v^{(m)}\left(0^{\prime}, c_{m-1}\right)\right| \leqq C(n, \bar{\lambda}, \bar{\Lambda}) c_{m-1}, \quad m_{0}<m \leqq m^{*} .
$$

The desired uniform estimate for the $b^{(m)}, m_{0}<m \leqq m^{*}$, follows immediately. By iterated use of the identity

$$
a^{(m)}+b^{(m)} \cdot\left(0^{\prime}, c_{m}\right)=a^{(m-1)}+b^{(m-1)} \cdot\left(0^{\prime}, c_{m-1}\right)+O\left(c_{m}-c_{m-1}\right),
$$

it similarly follows that

$$
\left|a^{(m)}+b^{(m)} \cdot\left(0^{\prime}, c_{m}\right)\right| \leqq C \quad m_{0}<m<m^{*} .
$$

Since the $b^{(m)}$ and $c_{m}$ are bounded this gives the desired uniform estimate for the $a^{(m)}, m_{0}<m \leqq m^{*}$. This completes our verification of (18), and we now turn to the estimate (17). We only verify this estimate for $x \in \bar{\Omega}_{m} \cap \frac{1}{2} \Omega, m_{0}<m \leqq m^{*}$. The verification is entirely similar for $x \in \Omega_{m} \cap \frac{1}{2} \Omega, m_{*} \leqq m<m_{0}$ (and it is trivial for $\left.x \in \Omega_{m_{0}} \cap \frac{1}{2} \Omega\right)$. We write $x=\left(x^{\prime}, x_{n}\right)$, and (since we suppose $\bar{\Omega}_{m} \cap \frac{1}{2} \Omega \neq \emptyset$ ) we a priori know that $0<c_{m_{0}} \leqq c_{m-1} \leqq x_{n} \leqq \min \left\{\frac{1}{2}, c_{m}\right\}$. Due to (20),

$$
\left|v\left(x^{\prime}, c_{m_{0}}\right)-p\left(x^{\prime}, c_{m_{0}}\right)\right| \leqq C\left(\left(c_{m_{0}}\right)^{2}+\left|x^{\prime}\right|^{2}\right) \leqq C|x|^{2} .
$$

By Taylor's expansion and Proposition 2.1,

$$
\begin{aligned}
& v\left(x^{\prime}, x_{n}\right)-v\left(x^{\prime}, c_{m_{0}}\right) \\
= & v^{(m)}\left(x^{\prime}, x_{n}\right)-v^{(m)}\left(x^{\prime}, c_{m-1}\right)+\sum_{j=m_{0}+1}^{m-1}\left[v^{(j)}\left(x^{\prime}, c_{j}\right)-v^{(j)}\left(x^{\prime}, c_{j-1}\right)\right] \\
= & \partial_{n} v^{(m)}\left(x^{\prime}, c_{m-1}\right)\left(x_{n}-c_{m-1}\right)+\sum_{j=m_{0}+1}^{m-1} \partial_{n} v^{(j)}\left(x^{\prime}, c_{j-1}\right)\left(c_{j}-c_{j-1}\right)+O\left(|x|^{2}\right) .
\end{aligned}
$$

Here we have used the fact that $\sum_{j=m_{0}+1}^{m-1}\left(c_{j}-c_{j-1}\right)^{2} \leqq\left[\sum_{j=m_{0}+1}^{m-1}\left(c_{j}-c_{j-1}\right)\right]^{2}=$ $\left(c_{m-1}-c_{m_{0}}\right)^{2}$. By the definition of $p(x)$,

$$
p\left(x^{\prime}, x_{n}\right)-p\left(x^{\prime}, c_{m_{0}}\right)=b_{n}^{(m)}\left(x_{n}-c_{m-1}\right)+\sum_{j=m_{0}+1}^{m-1} b_{n}^{(j)}\left(c_{j}-c_{j-1}\right) .
$$

From the exact same analysis that led to (25), we also get

$$
\left|b^{(j)}-\nabla v^{(j)}\left(x^{\prime}, c_{j-1}\right)\right| \leqq C(n, \bar{\lambda}, \bar{\Lambda})\left(\left|x^{\prime}\right|+c_{j-1}\right), \quad m_{0}<j \leqq m^{*} .
$$

By subtraction of the above identities for $v\left(x^{\prime}, x_{n}\right)-v\left(x^{\prime}, c_{m_{0}}\right)$ and $p\left(x^{\prime}, x_{n}\right)-$ $p\left(x^{\prime}, c_{m_{0}}\right)$, a small rearrangement, and subsequent use of the estimates (26) and (27), it follows that

$$
\left|v\left(x^{\prime}, x_{n}\right)-p\left(x^{\prime}, x_{n}\right)\right| \leqq C(n, \bar{\lambda}, \bar{\Lambda})|x|^{2} .
$$

Remark 2.3. We note that the constant $C$ in this corollary is independent of $l$; this is why the estimates (17) and (18) are only valid for $x \in \frac{1}{2} \Omega$ and $m_{*} \leqq m \leqq m^{*}$. If we dropped the insistence on independence of $l$, then the estimates (17) and (18) would trivially be satisfied for $x \in \Omega$ and $1 \leqq m \leqq l$. 


\section{A perturbation result}

Let $\Omega$ denote the $n$-dimensional cube $(-1,1)^{n}$, and let the "strips" $\Omega_{m}$ be as introduced in the previous section. The two sets of positive definite matrix functions $\mathcal{A}(\bar{\lambda}, \bar{\Lambda})$ and $\overline{\mathcal{A}}(\bar{\lambda}, \bar{\Lambda})$ are also as introduced earlier. In the introduction we briefly explained how Theorem 1.1 and Theorem 1.2 will be derived from Proposition 2.1 (or rather from Corollary 2.6) by a perturbation analysis. In this context we shall need a fairly basic lemma, which we state and prove below.

Lemma 3.1. Suppose $A \in \mathcal{A}(\bar{\lambda}, \bar{\Lambda})$ and $\bar{A} \in \overline{\mathcal{A}}(\bar{\lambda}, \bar{\Lambda})$. Suppose $g=\left(g_{1}, \cdots, g_{n}\right)$ $\in L^{q}(\Omega), h \in L^{q / 2}(\Omega)$ for some $q>n$, and suppose $\bar{G}=\left(\bar{G}_{1}, \cdots, \bar{G}_{n}\right)$ and $\bar{H}$ are constant on each of the strips $\Omega_{m}$. Let $u \in H^{1}(\Omega)$ be a solution to

$$
\partial_{i}\left(A_{i j} \partial_{j} u\right)=h+\partial_{i} g_{i} \text { in } \Omega,
$$

with

$$
\|u\|_{L^{\infty}(\Omega)} \leqq 1 .
$$

There exist positive constants $\gamma \leqq 1$ and $C$ (depending only on $q, n, \bar{\lambda}$ and $\bar{\Lambda}$ ) such that if

$$
\left(f_{\Omega}|A-\bar{A}|^{q} d x\right)^{1 / q} \leqq \varepsilon
$$

for some $\varepsilon>0$, then we may find a function $v \in H^{1}$ with

$$
\partial_{i}\left(\bar{A}_{i j}(x) \partial_{j} v\right)=\bar{H}+\partial_{i}\left(\bar{G}_{i}\right) \text { in } \frac{3}{4} \Omega,
$$

and

$$
\begin{aligned}
& \|u-v\|_{L^{\infty}\left(\frac{1}{2} \Omega\right)} \\
& \leqq C\left(\left[1+\|\bar{G}\|_{L^{\infty}(\Omega)}+\|\bar{H}\|_{L^{\infty}(\Omega)}\right] \varepsilon^{\gamma}+\|g-\bar{G}\|_{L^{q}(\Omega)}+\|h-\bar{H}\|_{L^{q / 2}(\Omega)}\right) .
\end{aligned}
$$

Proof. Since

$$
\partial_{i}\left(A_{i j}(x) \partial_{j} u\right)=h+\partial_{i} g_{i}(x) \quad \text { in } \Omega,
$$

it follows from interior De Giorgi-Nash estimates ([8], Theorem 8.24) that there exist $\gamma^{\prime}(n, q, \bar{\lambda}, \bar{\Lambda})$ in the range $0<\gamma^{\prime}(n, q, \bar{\lambda}, \bar{\Lambda})<1$ and $C(n, q, \bar{\lambda}, \bar{\Lambda})$ such that

$$
\begin{aligned}
\|u\|_{C \gamma^{\prime}\left(\overline{\left.\frac{3}{4} \Omega\right)} \leqq\right.} & C\left(\|u\|_{L^{\infty}(\Omega)}+\|\bar{G}\|_{L^{\infty}(\Omega)}\right. \\
& \left.+\|\bar{H}\|_{L^{\infty}(\Omega)}+\|g-\bar{G}\|_{L^{q}(\Omega)}+\|h-\bar{H}\|_{L^{q / 2}(\Omega)}\right) \\
\leqq & C\left(1+\|\bar{G}\|_{L^{\infty}(\Omega)}+\|\bar{H}\|_{L^{\infty}(\Omega)}\right. \\
& \left.+\|g-\bar{G}\|_{L^{q}(\Omega)}+\|h-\bar{H}\|_{L^{q / 2}(\Omega)}\right) \\
= & C(K(\bar{G}, \bar{H})+k(g, h, \bar{G}, \bar{H})) .
\end{aligned}
$$


Here we have, for later convenience, introduced the notation

$$
\begin{aligned}
K(\bar{G}, \bar{H}) & =1+\|\bar{G}\|_{L^{\infty}(\Omega)}+\|\bar{H}\|_{L^{\infty}(\Omega)}, \\
k(g, h, \bar{G}, \bar{H}) & =\|g-\bar{G}\|_{L^{q}(\Omega)}+\|h-\bar{H}\|_{L^{q / 2}(\Omega)} .
\end{aligned}
$$

Let $v$ denote the solution to

$$
\begin{aligned}
\partial_{i}\left(\bar{A}_{i j}(x) \partial_{j} v\right) & =\bar{H}+\partial_{i}\left(\bar{G}_{i}\right) \quad \text { in } \frac{3}{4} \Omega, \\
v & =u \quad \text { on } \partial\left(\frac{3}{4} \Omega\right) .
\end{aligned}
$$

By $L^{\infty}$ estimates (see [8], Theorem 8.16)

$$
\begin{aligned}
\|v\|_{L^{\infty}\left(\frac{3}{4} \Omega\right)} & \leqq\|u\|_{L^{\infty}\left(\partial\left(\frac{3}{4} \Omega\right)\right)}+C\left(\|\bar{G}\|_{L^{\infty}\left(\frac{3}{4} \Omega\right)}+\|\bar{H}\|_{L^{\infty}\left(\frac{3}{4} \Omega\right)}\right) \\
& \leqq C K(\bar{G}, \bar{H}) .
\end{aligned}
$$

It now follows from the global De Giorgi-Nash estimates ([8], Theorem 8.29) that, for some $\gamma$ and $\gamma^{\prime}$ such that $0<\gamma \leqq \gamma^{\prime}$,

$$
\begin{aligned}
\|v\|_{C^{\gamma}\left(\frac{3}{4} \Omega\right)} & \leqq C\left(\|u\|_{C^{\gamma^{\prime}}\left(\overline{\frac{3}{4} \Omega}\right)}+\|v\|_{L^{\infty}\left(\frac{3}{4} \Omega\right)}+\|\bar{G}\|_{L^{\infty}\left(\frac{3}{4} \Omega\right)}+\|\bar{H}\|_{L^{\infty}\left(\frac{3}{4} \Omega\right)}\right) \\
& \leqq C(K(\bar{G}, \bar{H})+k(g, h, \bar{G}, \bar{H})) .
\end{aligned}
$$

Based on this estimate and (28) we immediately get

$$
\|u-v\|_{L^{\infty}\left(\partial\left[\left(\frac{3}{4}-s\right) \Omega\right]\right)} \leqq C(K(\bar{G}, \bar{H})+k(g, h, \bar{G}, \bar{H})) s^{\gamma} .
$$

A slightly more involved argument, utilizing in particular Proposition 2.1, gives that

$$
\|\nabla v\|_{L^{\infty}\left(\left(\frac{3}{4}-s\right) \Omega\right)} \leqq C(K(\bar{G}, \bar{H})+k(g, h, \bar{G}, \bar{H})) s^{\gamma-1} .
$$

This latter argument goes as follows. For any fixed $\bar{x} \in\left(\frac{3}{4}-s\right) \Omega$ define

$$
w(x)=\frac{v(\bar{x}+s x)-v(\bar{x})}{s^{\gamma}}, \quad|x| \leqq 1 .
$$

It follows from the estimate (29) that $|w(x)| \leqq C(K(\bar{G}, \bar{H})+k(g, h, \bar{G}, \bar{H}))$ for all $|x| \leqq 1$. We also have that

$$
\partial_{x_{i}}\left(\bar{A}_{i j}(\bar{x}+s x) \partial_{x_{j}} w\right)=s^{2-\gamma} \bar{H}(\bar{x}+s x)+s^{1-\gamma} \partial_{x_{i}} \bar{G}_{i}(\bar{x}+s x) \quad \text { for }|x| \leqq 1,
$$

and so from Proposition 2.1 we conclude that

$$
|\nabla w(0)| \leqq C(K(\bar{G}, \bar{H})+k(g, h, \bar{G}, \bar{H})),
$$

or

$$
|\nabla v(\bar{x})| \leqq C(K(\bar{G}, \bar{H})+k(g, h, \bar{G}, \bar{H})) s^{\gamma-1},
$$


exactly as desired. A simple calculation gives

$$
\begin{aligned}
\partial_{i}\left(A_{i j}(x) \partial_{j}(u-v)\right)= & h-\bar{H}+\partial_{i}\left(g_{i}-\bar{G}_{i}\right)-\partial_{i}\left(\left(A_{i j}(x)-\bar{A}_{i j}(x)\right) \partial_{j} v\right), \\
& x \in \frac{3}{4} \Omega,
\end{aligned}
$$

and from this and the estimates (30) and (31) it follows, using Theorem 8.16 in [8] again, that

$$
\begin{aligned}
\|u-v\|_{L^{\infty}\left(\left(\frac{3}{4}-s\right) \Omega\right)} \leqq & \|u-v\|_{L^{\infty}\left(\partial\left[\left(\frac{3}{4}-s\right) \Omega\right]\right)} \\
& +C\left(\|g-\bar{G}\|_{L^{q}\left(\left(\frac{3}{4}-s\right) \Omega\right)}+\|h-\bar{H}\|_{L^{q / 2}\left(\left(\frac{3}{4}-s\right) \Omega\right)}\right. \\
& \left.+\left\|\left(A_{i j}(x)-\bar{A}_{i j}(x)\right) \partial_{j} v\right\|_{L^{q}\left(\left(\frac{3}{4}-s\right) \Omega\right)}\right) \\
\leqq & C\left([K(\bar{H}, \bar{G})+k(g, h, \bar{G}, \bar{H})] s^{\gamma}+k(g, h, \bar{G}, \bar{H})\right. \\
& \left.+\varepsilon[K(\bar{H}, \bar{G})+k(g, h, \bar{G}, \bar{H})] s^{\gamma-1}\right) .
\end{aligned}
$$

We note that $C$ depends on $q, n, \bar{\lambda}$ and $\bar{\Lambda}$, but is independent of $0<s \leqq 1 / 4$. We now pick $s$ equal to $\min \{\varepsilon, 1 / 4\}$ to get

$$
\|u-v\|_{L^{\infty}\left(\frac{1}{2} \Omega\right)} \leqq C\left(K(\bar{H}, \bar{G}) \varepsilon^{\gamma}+k(g, h, \bar{G}, \bar{H})\right) .
$$

This completes the proof of the lemma.

For any $0<s$ and any $1<p<\infty$ we introduce the norm

$$
\|h\|_{Y^{s, p}}:=\sup _{0<r \leqq 1} r^{1-s}\left(f_{r \Omega}|h|^{p}\right)^{1 / p} .
$$

For convenience we slightly redefine the indices $m_{*}$ and $m^{*}$

$$
\begin{aligned}
& m_{*}=\min \left\{m: \Omega_{m} \cap \frac{1}{4} \Omega \neq \emptyset\right\}=\min \left\{m:-\frac{1}{4}<c_{m}\right\}, \\
& m^{*}=\max \left\{m: \Omega_{m} \cap \frac{1}{4} \Omega \neq \emptyset\right\}=\max \left\{m: c_{m-1}<\frac{1}{4}\right\} .
\end{aligned}
$$

We are now ready to establish our main perturbation result.

Proposition 3.2. Suppose $A \in \mathcal{A}(\bar{\lambda}, \bar{\Lambda})$ and $\bar{A} \in \overline{\mathcal{A}}(\bar{\lambda}, \bar{\Lambda})$, relative to the hyperplanes $x_{n}=c_{m}, 0 \leqq m \leqq l+1$. Suppose $g=\left(g_{1}, \cdots, g_{n}\right) \in L^{q}(\Omega), h \in L^{q / 2}(\Omega)$ for some $q>n$, and suppose $\bar{G}=\left(\bar{G}_{1}, \cdots, \bar{G}_{n}\right)$ and $\bar{H}$ are constant on each of the strips $\Omega_{m}$. Let $0<\bar{\alpha}<1$, and let $u \in H^{1}(\Omega)$ denote a solution to

$$
\partial_{i}\left(A_{i j}(x) \partial_{j} u\right)=h+\partial_{i} g_{i} \quad \text { in } \Omega
$$

with

$$
\|u\|_{L^{\infty}(\Omega)} \leqq 1
$$


There exist constants $0<\sigma<\frac{1}{4}, \varepsilon_{0}>0$, and $C>0$ (depending on $n, q, \bar{\alpha}$, and the ellipticity constants $\bar{\lambda}, \bar{\Lambda}$ ) such that if

$$
\begin{gathered}
\|A-\bar{A}\|_{Y^{1+\bar{\alpha}, q}} \leqq \varepsilon_{0} \\
\|g-\bar{G}\|_{Y^{1+\bar{\alpha}, q}}+\|h-\bar{H}\|_{Y^{\bar{\alpha}, q / 2}} \leqq \varepsilon_{0}, \quad \text { and } \quad\|\bar{G}\|_{L^{\infty}(\Omega)}+\|\bar{H}\|_{L^{\infty}(\Omega)} \leqq 1
\end{gathered}
$$

then we may find a sequence of continuous, piecewise linear functions $p_{k}, k=$ $1,2, \ldots$,

$$
p_{k}(x)=a_{k}^{(m)}+b_{k}^{(m)} \cdot x, \quad x \in \Omega_{m}, m_{*} \leqq m \leqq m^{*},
$$

with

$$
\begin{aligned}
\left|a_{1}^{(m)}\right|+\left|b_{1}^{(m)}\right| & \leqq C \\
\left|a_{k}^{(m)}-a_{k-1}^{(m)}\right| & \leqq C\left[\left(\sigma^{k-1}\right)^{1+\bar{\alpha}}+\min \left\{\left|c_{m-1}\right|,\left|c_{m}\right|\right\}\left(\sigma^{k-1}\right)^{\bar{\alpha}}\right], \\
\left|b_{k}^{(m)}-b_{k-1}^{(m)}\right| & \leqq C\left(\sigma^{k-1}\right)^{\bar{\alpha}}, \\
b_{k}^{(m)} & =M^{(m)} b_{k}^{(m-1)}+\left(0^{\prime}, \frac{1}{\bar{A}_{n n}^{(m)}}\left[\bar{G}_{n}^{(m)}-\bar{G}_{n}^{(m-1)}\right]\right),
\end{aligned}
$$

for $m_{*} \leqq m \leqq m^{*}$, and such that

$$
\left\|u-p_{k}\right\|_{L^{\infty}\left(\sigma^{k} \Omega\right)} \leqq\left(\sigma^{k}\right)^{1+\bar{\alpha}}
$$

The limit $p(x)=\lim _{k \rightarrow \infty} p_{k}(x)$ exists for $x \in \frac{1}{4} \Omega$. It is a continuous, piecewise linear function with coefficients that are uniformly bounded by $C . p(\cdot)$ furthermore satisfies

$$
\partial_{i}\left(\bar{A}_{i j}(x) \partial_{j}\right) p=\partial_{i}\left(\bar{G}_{i}\right) \text { in } \frac{1}{4} \Omega,
$$

and

$$
|u(x)-p(x)| \leqq C|x|^{1+\bar{\alpha}}, \quad x \in \frac{1}{4} \Omega .
$$

Remark 3.1. From the uniform boundedness of the coefficients of $p(x)$ it follows immediately that $|p(x)-p(0)| \leqq C|x|$. The estimate (33) thus implies

$$
|u(x)-u(0)| \leqq|u(x)-p(x)|+|p(x)-p(0)|+|p(0)-u(0)| \leqq C|x| .
$$

Proof of Proposition 3.2. The existence of the piecewise linear functions, $p_{k}$, is established by induction. We first prove the existence of a $p_{1}$ with the required properties. From the first and second inequalities in the hypothesis (32) it immediately follows that $\|A-\bar{A}\|_{L^{q}(\Omega)} \leqq C \varepsilon_{0}$ and $\|g-\bar{G}\|_{L^{q}(\Omega)}+\|h-\bar{H}\|_{L^{q / 2}(\Omega)} \leqq C \varepsilon_{0}$. Therefore, according to Lemma 3.1, there exists a function $v \in H^{1}$, satisfying

$$
\partial_{i}\left(\bar{A}_{i j}(x) \partial_{j} v\right)=\bar{H}+\partial_{i}\left(\bar{G}_{i}\right) \quad \text { in } \frac{3}{4} \Omega,
$$

and with

$$
\|u-v\|_{L^{\infty}\left(\frac{1}{2} \Omega\right)} \leqq C \varepsilon_{0}^{\gamma} .
$$


According to the last estimate we also have

$$
\|v\|_{L^{\infty}\left(\frac{1}{2} \Omega\right)} \leqq C,
$$

and thus, in view of Corollary 2.6 (with $\Omega$ replaced by $\frac{1}{2} \Omega$ ) there exists a continuous, piecewise linear function

$$
p_{1}(x)=a_{1}^{(m)}+b_{1}^{(m)} \cdot x, \quad x \in \Omega_{m}, m_{*} \leqq m \leqq m^{*},
$$

with

$$
\begin{aligned}
\left|a_{1}^{(m)}\right|+\left|b_{1}^{(m)}\right| & \leqq C, \\
b_{1}^{(m)} & =M^{(m)} b_{1}^{(m-1)}+\left(0^{\prime}, \frac{1}{A_{n n}^{(m)}}\left[\bar{G}_{n}^{(m)}-\bar{G}_{n}^{(m-1)}\right]\right),
\end{aligned}
$$

and such that

$$
\left|v(x)-p_{1}(x)\right| \leqq C|x|^{2}, \quad x \in \frac{1}{4} \Omega .
$$

Here $M^{(m)}$ is the same matrix as earlier. By a combination of the estimates for $u-v$ and $v-p_{1}$ we get

$$
\left|u(x)-p_{1}(x)\right| \leqq C \varepsilon_{0}^{\gamma}+C|x|^{2}, \quad x \in \frac{1}{4} \Omega .
$$

Now select $\sigma<\frac{1}{4}$ sufficiently small, so that

$$
C \sigma^{2}=C \sigma^{1-\bar{\alpha}} \sigma^{1+\bar{\alpha}} \leqq \frac{1}{2} \sigma^{1+\bar{\alpha}},
$$

it then follows from (35) that

$$
\left|u(x)-p_{1}(x)\right| \leqq C \varepsilon_{0}^{\gamma}+\frac{1}{2} \sigma^{1+\bar{\alpha}}, \quad x \in \sigma \Omega .
$$

Select $\varepsilon_{0}$ sufficiently small that $C \varepsilon_{0}^{\gamma}<\frac{1}{2} \sigma^{1+\bar{\alpha}}$, and altogether we have

$$
\left\|u-p_{1}\right\|_{L^{\infty}(\sigma \Omega)} \leqq \sigma^{1+\bar{\alpha}}
$$

which is exactly the estimate $\left(P_{1}\right)$.

Suppose we have established the existence of $p_{1}, \ldots, p_{k}$ with the required properties; to complete the induction proof we must now construct an appropriate $p_{k+1}$. It follows from the induction hypotheses that

$$
\max _{1 \leqq i \leqq k, m_{*} \leqq m \leqq m^{*}}\left\{\left|a_{i}^{(m)}\right|,\left|b_{i}^{(m)}\right|\right\} \leqq C .
$$

Consider the function

$$
W(x)=\frac{u\left(\sigma^{k} x\right)-p_{k}\left(\sigma^{k} x\right)}{\left(\sigma^{k}\right)^{1+\bar{\alpha}}}, \quad x \in \Omega .
$$

According to the induction hypotheses this function satisfies

$$
\|W\|_{L^{\infty}(\Omega)} \leqq 1
$$


and

$$
\begin{aligned}
\partial_{i}\left(A_{i j}\left(\sigma^{k} x\right) \partial_{j} W\right) & =\frac{h\left(\sigma^{k} x\right)}{\left(\sigma^{k}\right)^{\bar{\alpha}-1}}+\frac{\partial_{i}\left[g_{i}\left(\sigma^{k} x\right)\right]}{\left(\sigma^{k}\right)^{\bar{\alpha}}}-\frac{1}{\left(\sigma^{k}\right)^{1+\bar{\alpha}}} \partial_{i}\left(A_{i j}\left(\sigma^{k} x\right) \partial_{j} p_{k}\left(\sigma^{k} x\right)\right) \\
& =h_{k}(x)+\partial_{i} g_{k, i}(x),
\end{aligned}
$$

with $h_{k}$ and $g_{k, i}$ given by

$$
h_{k}(x)=\frac{h\left(\sigma^{k} x\right)}{\left(\sigma^{k}\right)^{\bar{\alpha}-1}}
$$

and

$g_{k, i}(x)=\frac{1}{\left(\sigma^{k}\right)^{\bar{\alpha}}}\left(g_{i}\left(\sigma^{k} x\right)-\left[A_{i j}\left(\sigma^{k} x\right)-\bar{A}_{i j}\left(\sigma^{k} x\right)\right] b_{k, j}^{(m)}-\bar{G}_{i}^{(m)}\right) \quad$ in $\sigma^{-k} \Omega_{m}$.

A simple calculation, using the two first inequalities of the hypothesis (32), gives that

$$
\begin{gathered}
\left\|\frac{1}{\left(\sigma^{k}\right)^{\bar{\alpha}}}\left[A_{i j}\left(\sigma^{k} \cdot\right)-\bar{A}_{i j}\left(\sigma^{k} \cdot\right)\right]\right\|_{L^{q}(\Omega)} \leqq C \varepsilon_{0}, \quad \text { and } \\
\left\|g_{k}\right\|_{L^{q}(\Omega)}+\left\|h_{k}-\bar{H}_{k}\right\|_{L^{q / 2}(\Omega)} \leqq C \varepsilon_{0},
\end{gathered}
$$

where

$$
\bar{H}_{k}=\frac{\bar{H}\left(\sigma^{k} x\right)}{\left(\sigma^{k}\right)^{\bar{\alpha}-1}} .
$$

From Lemma 3.1 we now infer the existence of a solution to

$$
\partial_{i}\left(\bar{A}_{i j}\left(\sigma^{k} x\right) \partial_{j} Z\right)=\bar{H}_{k} \quad \text { in } \frac{3}{4} \Omega,
$$

with the property that

$$
\|W-Z\|_{L^{\infty}\left(\frac{1}{2} \Omega\right)} \leqq C \varepsilon_{0}^{\gamma} .
$$

According to the last estimate we also have

$$
\|Z\|_{L^{\infty}\left(\frac{1}{2} \Omega\right)} \leqq C
$$

and thus, in view of Corollary 2.6, there exists a continuous, piecewise linear function $p$

$$
p(x)=a^{(m)}+b^{(m)} \cdot x, \quad x \in \sigma^{-k} \Omega_{m}, \quad m_{*} \leqq m \leqq m^{*},
$$

with

$$
\begin{aligned}
b^{(m)} & =M^{(m)} b^{(m-1)}, \\
a^{(m)}+b^{(m)} \cdot\left(0^{\prime}, \sigma^{-k} c_{m-1}\right) & =a^{(m-1)}+b^{(m-1)} \cdot\left(0^{\prime}, \sigma^{-k} c_{m-1}\right),
\end{aligned}
$$

and such that

$$
|Z(x)-p(x)| \leqq C|x|^{2}, \quad x \in \frac{1}{4} \Omega
$$


The function $p$ is initially (according to Corollary 2.6) only defined on those $\Omega_{m}$ for which $\sigma^{-k} \Omega_{m} \cap \frac{1}{4} \Omega \neq \emptyset$. We extend $p$ to all $\Omega_{m}, m_{*} \leqq m \leqq m^{*}$, by means of the identities (37). Since $\sigma^{-k} \Omega_{m_{0}} \cap \frac{1}{4} \Omega \neq \emptyset$ Corollary 2.6 asserts that

$$
\left|a^{\left(m_{0}\right)}\right|+\left|b^{\left(m_{0}\right)}\right| \leqq C,
$$

even though it does not provide similarly $l$-independent bounds for all $m_{*} \leqq m \leqq$ $m^{*}$. By a combination of the estimates for $W-Z$ and $Z-p$ we get

$$
|W(x)-p(x)| \leqq C \varepsilon_{0}^{\gamma}+C|x|^{2}, \quad x \in \frac{1}{4} \Omega .
$$

With our choice of $\sigma$ and $\varepsilon_{0}$ we conclude (by repetition of an earlier argument) that

$$
|W(x)-p(x)| \leqq \sigma^{1+\bar{\alpha}}, \quad x \in \sigma \Omega,
$$

and thus,

$$
\left|u\left(\sigma^{k} x\right)-p_{k}\left(\sigma^{k} x\right)-\left(\sigma^{k}\right)^{1+\bar{\alpha}} p(x)\right| \leqq\left(\sigma^{k+1}\right)^{1+\bar{\alpha}}, \quad x \in \sigma \Omega .
$$

It follows immediately that $p_{k+1}(y):=p_{k}(y)+\left(\sigma^{k}\right)^{1+\bar{\alpha}} p\left(y / \sigma^{k}\right)$ satisfies the estimate $\left(P_{k+1}\right)$. We shall only verify the estimates concerning the coefficients of $p_{k+1}$ for $m_{0} \leqq m \leqq m^{*}$. The verification for $m_{*} \leqq m<m_{0}$ proceeds similarly, but is left to the reader. Since $p_{k+1}$ (as well as $p_{k}$ ) satisfies $\partial_{i}\left(\bar{A}_{i j}(x) \partial_{j}\right) p_{k+1}=$ $\partial_{i}\left(\bar{G}_{i}\right)$ in $\frac{1}{4} \Omega$, we have

$$
\begin{aligned}
b_{k+1}^{(m)}-b_{k}^{(m)} & =M^{(m)}\left[b_{k+1}^{(m-1)}-b_{k}^{(m-1)}\right] \\
& =\ldots=\left[N^{(m)}\right]^{-1} N^{\left(m_{0}\right)}\left[b_{k+1}^{\left(m_{0}\right)}-b_{k}^{\left(m_{0}\right)}\right] \quad m_{0} \leqq m \leqq m^{*} .
\end{aligned}
$$

From (38) and the definition of $p_{k+1}$ we conclude that

$$
\left|b_{k+1}^{\left(m_{0}\right)}-b_{k}^{\left(m_{0}\right)}\right|=\left(\sigma^{k}\right)^{\bar{\alpha}}\left|b^{\left(m_{0}\right)}\right| \leqq C\left(\sigma^{k}\right)^{\bar{\alpha}} .
$$

This estimate, in combination with (39), leads to

$$
\left|b_{k+1}^{(m)}-b_{k}^{(m)}\right| \leqq C\left(\sigma^{k}\right)^{\bar{\alpha}}, \quad m_{0} \leqq m \leqq m^{*},
$$

with $C=C(n, q, \bar{\alpha}, \bar{\lambda}, \bar{\Lambda})$. To establish the bounds concerning the $a_{k+1}$ 's we first insert $y=0$ into the definition of $p_{k+1}$. This yields

$$
a_{k+1}^{\left(m_{0}\right)}-a_{k}^{\left(m_{0}\right)}=\left(\sigma^{k}\right)^{1+\bar{\alpha}} a^{\left(m_{0}\right)},
$$

and so, by means of (38), we get

$$
\left|a_{k+1}^{\left(m_{0}\right)}-a_{k}^{\left(m_{0}\right)}\right| \leqq C\left(\sigma^{k}\right)^{1+\bar{\alpha}} .
$$

Using the already established estimate for $b_{k+1}^{(m)}-b_{k}^{(m)}$ and the continuity of $p_{k+1}-$ $p_{k}\left(\right.$ at $\left.x_{n}=c_{m-1}\right)$

$$
\begin{aligned}
& a_{k+1}^{(m)}-a_{k}^{(m)}+\left(b_{k+1}^{(m)}-b_{k}^{(m)}\right) \cdot\left(0^{\prime}, c_{m-1}\right) \\
& =a_{k+1}^{(m-1)}-a_{k}^{(m-1)}+\left(b_{k+1}^{(m-1)}-b_{k}^{(m-1)}\right) \cdot\left(0^{\prime}, c_{m-2}\right)+O\left(\left(\sigma^{k}\right)^{\bar{\alpha}}\left(c_{m-1}-c_{m-2}\right),\right.
\end{aligned}
$$


for $m_{0}+1<m \leqq m^{*}$. By iteration of this identity we get

$$
\begin{aligned}
& a_{k+1}^{(m)}-a_{k}^{(m)}+\left(b_{k+1}^{(m)}-b_{k}^{(m)}\right) \cdot\left(0^{\prime}, c_{m-1}\right) \\
& =a_{k+1}^{\left(m_{0}+1\right)}-a_{k}^{\left(m_{0}+1\right)}+\left(b_{k+1}^{\left(m_{0}+1\right)}-b_{k}^{\left(m_{0}+1\right)}\right) \cdot\left(0^{\prime}, c_{m_{0}}\right)+O\left(\left(\sigma^{k}\right)^{\bar{\alpha}}\left(c_{m-1}-c_{m_{0}}\right)\right),
\end{aligned}
$$

for $m_{0}<m \leqq m^{*}$. Since

$$
\begin{aligned}
& a_{k+1}^{\left(m_{0}+1\right)}-a_{k}^{\left(m_{0}+1\right)}+\left(b_{k+1}^{\left(m_{0}+1\right)}-b_{k}^{\left(m_{0}+1\right)}\right) \cdot\left(0^{\prime}, c_{m_{0}}\right) \\
& \quad=a_{k+1}^{\left(m_{0}\right)}-a_{k}^{\left(m_{0}\right)}+\left(b_{k+1}^{\left(m_{0}\right)}-b_{k}^{\left(m_{0}\right)}\right) \cdot\left(0^{\prime}, c_{m_{0}}\right) \\
& \quad=O\left(\left(\sigma^{k}\right)^{1+\bar{\alpha}}+\left(\sigma^{k}\right)^{\bar{\alpha}} c_{m_{0}}\right),
\end{aligned}
$$

it now follows that

$$
\left|a_{k+1}^{(m)}-a_{k}^{(m)}+\left(b_{k+1}^{(m)}-b_{k}^{(m)}\right) \cdot\left(0^{\prime}, c_{m-1}\right)\right| \leqq C\left[\left(\sigma^{k}\right)^{1+\bar{\alpha}}+c_{m-1}\left(\sigma^{k}\right)^{\bar{\alpha}}\right],
$$

for $m_{0}<m \leqq m^{*}$. After insertion of the bound for $b_{k+1}^{(m)}-b_{k}^{(m)}$ we get

$$
\left|a_{k+1}^{(m)}-a_{k}^{(m)}\right| \leqq C\left[\left(\sigma^{k}\right)^{1+\bar{\alpha}}+c_{m-1}\left(\sigma^{k}\right)^{\bar{\alpha}}\right], \quad m_{0}<m \leqq m^{*} .
$$

This concludes the verification of the estimates concerning the coefficients of $p_{k+1}$. The induction proof of the existence of the sequence $p_{1}, p_{2}, \ldots$ is complete.

Using the estimates for the coefficients of $p_{k}(x)$ it is now (by summation of a telescoping sum) easy to see that $p(x)=\lim _{k \rightarrow \infty} p_{k}(x)$ exists for $x \in \frac{1}{4} \Omega$, and that it satisfies the same differential equation as the $p_{k}$ 's. From the estimates for the coefficients for $p_{k}$ it also follows that

$$
\left\|p_{k}-p\right\|_{L^{\infty}\left(\sigma^{k} \Omega\right)} \leqq C\left(\sigma^{k}\right)^{1+\bar{\alpha}},
$$

and thus, by combination with $\left(P_{k}\right)$,

$$
\|u-p\|_{L^{\infty}\left(\sigma^{k} \Omega\right)} \leqq C\left(\sigma^{k}\right)^{1+\bar{\alpha}} .
$$

This latter estimate immediately implies (33).

\section{A weakened version of Theorem 1.1.}

We return to the same notation as that used in the statement of the main theorems in the introduction to this paper. Let us make a few simplifying assumptions.

(A) Suppose all but one of the domains, $D_{m}$, are convex. We refer to these as the inhomogeneities. In the context of the two-dimensional example we discussed in the introduction these represent the "fiber cross sections".

(B) Suppose the coefficient matrix, $A$, is constant in each of the domains $D_{m}$, $1 \leqq m \leqq L$, and suppose $h=g=0$.

At the same time we restrict our goal to establishing a uniform estimate for the gradient of $u$. The resulting weakened theorem reads as follows. 


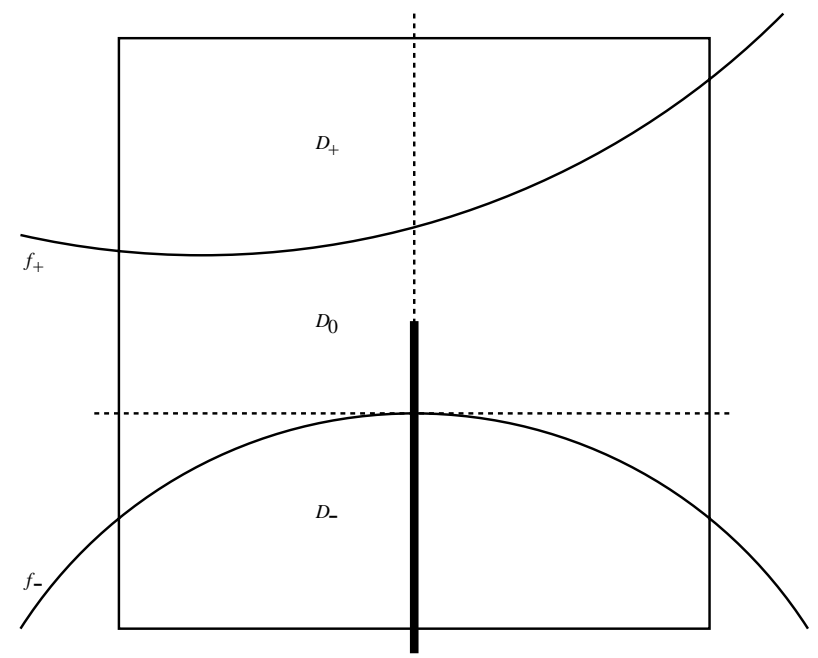

Fig. 1. The local situation. The bold vertical line represent the points of the form $\bar{x}=$ $\left(0^{\prime}, x_{n}\right)$, for which the origin is the closest point on the curves $\left\{\left(x^{\prime}, x_{n}\right): x_{n}=f_{ \pm}\left(x^{\prime}\right)\right.$, $\left.\left|x^{\prime}\right| \leqq 1\right\}$.

Theorem 4.1. Let the assumptions be as in Theorem 1.1, with the addition of (A) and (B) above. There exists a constant $C$ depending only on $D, n, \alpha, \varepsilon, \bar{\lambda}, \bar{\Lambda}$, and the $C^{1, \alpha}$ modulus of $\cup_{m=1}^{L} \partial D_{m}$, such that if $u \in H^{1}(D)$ is a solution to

$$
\partial_{i}\left(A_{i j} \partial_{j} u\right)=0, \quad \text { in } D,
$$

then

$$
\|\nabla u\|_{L^{\infty}\left(D_{\varepsilon}\right)} \leqq C\|u\|_{L^{\infty}(D)},
$$

where $D_{\varepsilon}=\{x \in D \mid \operatorname{dist}(x, \partial D)>\varepsilon\}$.

Since the $L^{\infty}$ estimate, referred to earlier (Theorem 8.16 in [8]) already verifies that $\|u\|_{L^{\infty}(\Omega)} \leqq\|u\|_{L^{\infty}(\partial \Omega)}$ the estimate in Theorem 4.1 immediately establishes an interior uniform bound for the gradient of $u$ in terms of the boundary data.

The geometric assumption, (A), guarantees that given any $\bar{x} \in D_{\varepsilon}$ there exists a cubic neighborhood $\bar{x}+(-c, c)^{n}$ such that this neighborhood overlaps with at most three of the domains $D_{m}$. The essential feature is that $c$, the size of this cube, depends on $\varepsilon, n$ and the $C^{1, \alpha}$ modulus of $\cup_{m=1}^{L} \partial D_{m}$, but is independent of $\bar{x}$. We may suppose the cube $\bar{x}+(-c, c)^{n}$ overlaps with exactly three of the domains (if not, we may simply create fictitious domain(s) without any discontinuity in the coefficient). We may also suppose that $\bar{x}$ has a (Euclidean distance) nearest point on $\cup \partial D_{m}$ which is a distance strictly smaller than $c / 2$ from $\bar{x}$ (if not we simply decrease $c$ and create fictitous domains). This nearest point is denoted $\bar{y}$. We may translate the origin of our coordinate system to $\bar{y}$ and rotate the coordinate system so that the $n$ 'th axis is normal to that $\partial D_{m}$ on which $\bar{y}$ lies. Considering the cube $Q=(-d, d)^{n}$ with $d=c / 2 \sqrt{n}$ we now obtain the situation depicted in Fig. 1. $Q$ intersects three of the domains $D_{m}$, we refer to the corresponding 
intersections as $D_{-}, D_{0}$ and $D_{+}$. There exist two $C^{1, \alpha}$ functions $f_{-}$and $f_{+}$with $-d \leqq f_{-}\left(x^{\prime}\right)<f_{+}\left(x^{\prime}\right) \leqq d, x^{\prime} \in[-d, d]^{n-1}$, such that $D_{-}, D_{0}$ and $D_{+}$are given by

$$
\begin{aligned}
& D_{-}=\left\{\left(x^{\prime}, x_{n}\right) \in(-d, d)^{n}:-d<x_{n}<f_{-}\left(x^{\prime}\right)\right\}, \\
& D_{0}=\left\{\left(x^{\prime}, x_{n}\right) \in(-d, d)^{n}: f_{-}\left(x^{\prime}\right)<x_{n}<f_{+}\left(x^{\prime}\right)\right\}, \\
& D_{+}=\left\{\left(x^{\prime}, x_{n}\right) \in(-d, d)^{n}: f_{+}\left(x^{\prime}\right)<x_{n}<d\right\} .
\end{aligned}
$$

The origin lies on the graph of one of the functions $f_{ \pm}$. We suppose it lies on the graph of $f_{-}$, i.e.,

$$
f_{-}\left(0^{\prime}\right)=0 \text {. }
$$

The $n$ 'th axis is normal to $\cup \partial D_{m}$ at the origin, i.e.,

$$
\nabla^{\prime} f_{-}\left(0^{\prime}\right)=0 \text {. }
$$

Since the origin is the (Euclidean distance) closest point to $\bar{x}$ on $\cup \partial D_{m}$, and since the $n$ 'th axis is normal to $\cup \partial D_{m}$ at the origin, $\bar{x}$ has the form $\bar{x}=\left(0^{\prime}, x_{n}\right)$. The fact that the boundary between $D_{0}$ and $D_{+}$is also the graph of a function follows by decreasing $d=c / 2 \sqrt{n}$, if necessary (exactly how much of a decrease is needed depends on $n$ and the $C^{1, \alpha}$ modulus of $\cup_{m=1}^{L} \partial D_{m}$, but is independent of $\bar{x}$ ). We could have allowed that $f_{-} \leqq f_{+}$(as opposed to the strict inequality $f_{-}<f_{+}$) because if this were the case, we simply replace $f_{+}$by $f_{+}+\delta$, and since the estimates we obtain are independent of $\delta$ we may then subsequently pass to the limit $\delta=0$.

We denote the constant coefficient matrices corresponding to $D_{-}, D_{0}$ and $D_{+}$ by $A^{(-)}, A^{(0)}$ and $A^{(+)}$respectively, and we introduce the notation

$$
A(x)= \begin{cases}A^{(+)} & x \in D_{+}, \\ A^{(0)} & x \in D_{0}, \\ A^{(-)} & x \in D_{+} .\end{cases}
$$

For simplicity of notation we from now on assume that $d=1$. In order to prove Theorem 4.1 it clearly suffices to prove the following proposition.

Proposition 4.2. Let $A(x), x \in \Omega=(-1,1)^{n}$ be the coefficient defined above for some $f_{ \pm} \in C^{1, \alpha}\left(\left|x^{\prime}\right| \leqq 1\right), 0<\alpha<1$, and let $u \in H^{1}(\Omega) \cap L^{\infty}(\Omega)$ denote a solution to

$$
\partial_{i}\left(A_{i j}(x) \partial_{j} u\right)=0 \text { in } \Omega .
$$

There exists a constant $C$ (depending on $n, \alpha,\left\|f_{ \pm}\right\|_{C^{1, \alpha}\left([-1,1]^{n-1}\right)}$ and the ellipticity constants $\bar{\lambda}, \bar{\Lambda})$ such that

$$
|\nabla u(\bar{x})| \leqq C\|u\|_{L^{\infty}(\Omega)}
$$

for any point $\bar{x}$ which has the form $\bar{x}=\left(0^{\prime}, x_{n}\right)$ and for which the origin is the closest point on the curves $\left\{\left(x^{\prime}, x_{n}\right): x_{n}=f_{ \pm}\left(x^{\prime}\right), \quad\left|x^{\prime}\right| \leqq 1\right\}$. 
The proof of Proposition 4.2 is based on Proposition 3.2 and a simple rescaling argument. To formulate the rescaling lemma we need some additional notation. Corresponding to the matrix-valued function $A$ we introduce, for any $r_{0} \leqq 1$,

$$
A_{r_{0}}(x)=A\left(r_{0} x\right), \quad x \in \Omega=(-1,1)^{n},
$$

We also introduce the matrix-valued function $\bar{A}_{r_{0}}(x), x \in \Omega=(-1,1)^{n}$,

$$
\bar{A}_{r_{0}}(x)=\left\{\begin{array}{lll}
A^{(+)} & \left|x^{\prime}\right|<1, & r_{0}^{-1} f_{+}(0)<x_{n}<1, \\
A^{(0)} & \left|x^{\prime}\right|<1, & r_{0}^{-1} f_{-}(0)<x_{n}<r_{0}^{-1} f_{+}(0), \\
A^{(-)} & \left|x^{\prime}\right|<1, & -1<x_{n}<r_{0}^{-1} f_{+}(0) .
\end{array}\right.
$$

The function $\bar{A}_{r_{0}}$ is in $\overline{\mathcal{A}}(\bar{\lambda}, \bar{\Lambda})$ (relative to the hyperplanes $\left.x_{n}=r_{0}^{-1} f_{ \pm}(0)\right)$.

Lemma 4.3. Let $A_{r_{0}}$ and $\bar{A}_{r_{0}}$ be defined by (40) and (41), and suppose $q \geq n$. Given any $\varepsilon_{0}>0$, there exists a positive constant $r_{0}$ depending on $n, q, \varepsilon_{0}, \alpha, \bar{\lambda}, \bar{\Lambda}$ and $\left\|f_{ \pm}\right\|_{C^{1, \alpha}\left([-1,1]^{n-1}\right)}$, such that

$$
\left(f_{r \Omega}\left|A_{r_{0}}(x)-\bar{A}_{r_{0}}(x)\right|^{q} d x\right)^{1 / q} \leqq \varepsilon_{0} r^{\frac{\alpha}{(\alpha+1) q}}, \quad \forall 0<r \leqq 1 .
$$

Proof. Since $f_{ \pm} \in C^{1, \alpha}\left(\left|x^{\prime}\right| \leqq 1\right)$, we have

$$
f_{ \pm}\left(x^{\prime}\right)=f_{ \pm}\left(0^{\prime}\right)+\nabla f_{ \pm}\left(0^{\prime}\right) x^{\prime}+O\left(\left|x^{\prime}\right|^{1+\alpha}\right),
$$

where $\left|O\left(\left|x^{\prime}\right|^{1+\alpha}\right)\right| \leqq C\left|x^{\prime}\right|^{1+\alpha}$ for some $C$ depending only on the $C^{1, \alpha}$ norm of $f_{ \pm}$. We also know that $f_{-}\left(x^{\prime}\right)<f_{+}\left(x^{\prime}\right)$, for $\left|x^{\prime}\right| \leqq 1, f_{-}\left(0^{\prime}\right)=0$, and $\nabla f_{-}\left(0^{\prime}\right)=0$, so

$$
f_{+}\left(0^{\prime}\right)+\nabla f_{+}\left(0^{\prime}\right) x^{\prime} \geqq-C\left|x^{\prime}\right|^{1+\alpha}, \quad\left|x^{\prime}\right| \leqq 1 .
$$

For convenience we now introduce the notation $\delta=f_{+}\left(0^{\prime}\right)$. It follows from the last inequality that

$$
\left|\nabla f_{+}\left(0^{\prime}\right)\right| \leqq C\left[f_{+}\left(0^{\prime}\right)\right]^{\frac{\alpha}{\alpha+1}}=C \delta^{\frac{\alpha}{\alpha+1}}
$$

For $0<r \leqq 1$, a simple calculation gives

$$
\left(f_{r \Omega}\left|A_{r_{0}}(x)-\bar{A}_{r_{0}}(x)\right|^{q} d x\right)^{1 / q}=\left(f_{s \Omega}\left|A(x)-\bar{A}_{r_{0}}\left(x / r_{0}\right)\right|^{q} d x\right)^{1 / q}
$$

with $s=r_{0} r \in\left(0, r_{0}\right)$. It is easy to see that there exists a constant, $\hat{c}>0$, depending only on the $C^{1}$ norm of $f_{+}$so that

$$
\left|\left(x^{\prime}, f_{+}\left(x^{\prime}\right)\right)\right| \geqq \hat{c} \delta
$$


for all $\left|x^{\prime}\right| \leqq 1$. For $r<\hat{c} \delta / r_{0}(s<\hat{c} \delta)$ we therefore estimate

$$
\begin{aligned}
\left(f_{s \Omega}\left|A(x)-\bar{A}_{r_{0}}\left(x / r_{0}\right)\right|^{q} d x\right)^{1 / q} & \leqq C\left(s^{-n} \int_{\left|x^{\prime}\right|<s}\left|f_{-}\left(x^{\prime}\right)\right| d x^{\prime}\right)^{1 / q} \\
& \leqq C\left(s^{-n} \int_{\left|x^{\prime}\right|<s}\left|x^{\prime}\right|^{1+\alpha} d x^{\prime}\right)^{1 / q} \\
& \leqq C s^{\alpha / q} \leqq C r_{0}^{\alpha / q} r^{\alpha / q}
\end{aligned}
$$

On the other hand, for $r \geqq \hat{c} \delta / r_{0}$, we get, using (42) and (43), that

$$
\begin{aligned}
\left(f_{s \Omega} \mid\right. & \left.A(x)-\left.\bar{A}_{r_{0}}\left(x / r_{0}\right)\right|^{q} d x\right)^{1 / q} \\
& \leqq C\left(s^{-n} \int_{\left|x^{\prime}\right|<s}\left(\left|f_{+}\left(x^{\prime}\right)-f_{+}\left(0^{\prime}\right)\right|+\left|f_{-}\left(x^{\prime}\right)\right|\right) d x^{\prime}\right)^{1 / q} \\
& \leqq C\left(s^{-n} \int_{\left|x^{\prime}\right|<s}\left(\delta^{\frac{\alpha}{\alpha+1}}\left|x^{\prime}\right|+\left|x^{\prime}\right|^{1+\alpha}\right) d x^{\prime}\right)^{1 / q} \\
& \leqq C\left(\delta^{\frac{\alpha}{(\alpha+1) q}}+s^{\frac{\alpha}{q}}\right) \leqq C r_{0}^{\frac{\alpha}{(\alpha+1) q}} r^{\frac{\alpha}{(\alpha+1) q}} .
\end{aligned}
$$

A combination of the estimates above yields

$$
\left(f_{r \Omega}\left|A_{r_{0}}(x)-\bar{A}_{r_{0}}(x)\right|^{q} d x\right)^{1 / q} \leqq C r_{0}^{\frac{\alpha}{(\alpha+1) q}} r^{\frac{\alpha}{(\alpha+1) q}} .
$$

We now simply choose $r_{0}$, so that $C r_{0}^{\frac{\alpha}{(\alpha+1) q}}=\varepsilon_{0}$, and the lemma follows.

Proof of Proposition 4.2. Let $\varepsilon_{0}$ be as in Proposition 3.2 and let $r_{0}$ be as in Lemma 4.3 (corresponding to this $\varepsilon_{0}$ ). The coefficient matrices $A_{r_{0}}$ and $\bar{A}_{r_{0}}$ are as defined in (40) and in (41). Define $w(x)=u\left(r_{0} x\right) /\|u\|_{L^{\infty}(\Omega)}, x \in \Omega$. The function $w \in$ $H^{1}(\Omega)$ satisfies

$$
\partial_{i}\left(\left(A_{r_{0}}\right)_{i j} \partial_{j} w\right)=0 \quad \text { in } \Omega
$$

and

$$
\|w\|_{L^{\infty}(\Omega)} \leqq 1
$$

To prove that $|\nabla u(\bar{x})| \leqq C\|u\|_{L^{\infty}(\Omega)}$ for all $\bar{x}$ which have the form $\bar{x}=\left(0, x_{n}\right)$ and for which the origin is the closest points on the curves $\left\{\left(x^{\prime}, x_{n}\right): x_{n}=f_{ \pm}\left(x^{\prime}\right)\right\}$ it clearly suffices to consider only $|\bar{x}|_{\infty}<r_{0} / 6$. If $|\bar{x}|_{\infty} \geqq r_{0} / 6$, then we are clearly a fixed distance away from the discontinuities in the coefficient, and classical elliptic interior estimates immediately give that $|\nabla u(\bar{x})| \leqq C\|u\|_{L^{\infty}(\Omega)}$. We also note that a bound for $\nabla w(x)$ immediately leads to a bound for $\nabla u\left(r_{0} x\right)$. In order to complete the proof of Proposition 4.2 it thus suffices to prove that

$$
|\nabla w(\tilde{x})| \leqq C, \quad \tilde{x} \in \frac{1}{6} \Omega
$$


for any $\tilde{x} \in \Omega$ which has the form $\tilde{x}=\left(0^{\prime}, x_{n}\right)$ and for which the origin is the closest points on the curves $\left\{\left(x^{\prime}, x_{n}\right): x_{n}=r_{0}^{-1} f_{ \pm}\left(r_{0} x^{\prime}\right)\right\}$. By a combination of Proposition 3.2 (Remark 3.1) and Lemma 4.3 we obtain the estimate

$$
|w(x)-w(0)| \leqq C|x|, \quad x \in \frac{1}{4} \Omega .
$$

For $\tilde{x}=0$ the estimate (44) follows immediately from (45). For $\tilde{x} \neq 0$, consider the function

$$
\hat{w}(y)=\frac{w\left(\tilde{x}+\frac{1}{2}|\tilde{x}| y\right)-w(0)}{|\tilde{x}|}, \quad|y| \leqq 1 .
$$

Since $\tilde{x}+\frac{1}{2}|\tilde{x}| y \in \frac{1}{4} \Omega$ it follows from (45) that

$$
|\hat{w}(y)| \leqq C, \quad|y| \leqq 1 .
$$

Since $\tilde{x}+\frac{1}{2}|\tilde{x}| y$ never touches the graphs of $r_{0}^{-1} f_{ \pm}\left(r_{0} x^{\prime}\right)$, due to the form of $\tilde{x}, \hat{w}$ satisfies a constant coefficient equation. Therefore, using classical elliptic estimates we have

$$
|\nabla w(\tilde{x})|=2|\nabla \hat{w}(0)| \leqq C .
$$

Proposition 4.2 is thus established.

\section{Interior estimates}

We now proceed to prove the full version of Theorem 1.1. For this purpose we first consider a (local) problem when the cube $\Omega=(-1,1)^{n}$ is divided into $l+1$ subdomains by $l C^{1, \alpha}$ curves. Let $0<\alpha<1$, and let $f_{1}, \cdots, f_{l}$ be $l$ functions in $C^{1, \alpha}\left(\left|x^{\prime}\right| \leqq 1\right)$ satisfying

$$
-1<f_{1}\left(x^{\prime}\right)<f_{2}\left(x^{\prime}\right)<\cdots<f_{l}\left(x^{\prime}\right)<1 \text { for all } x^{\prime} \in[-1,1]^{n-1} .
$$

We suppose

$$
f_{m_{0}-1}\left(0^{\prime}\right) \leqq 0<f_{m_{0}}\left(0^{\prime}\right), \quad \text { and }\left|\left(0^{\prime}, f_{m_{0}-1}\left(0^{\prime}\right)\right)\right|_{2}=\min _{1 \leqq m \leqq l} \min _{\left|x^{\prime}\right| \leqq 1} \mid\left(x^{\prime},\left.f_{m}\left(x^{\prime}\right)\right|_{2} .\right.
$$

Except when $f_{m_{0}-1}\left(0^{\prime}\right)=0$ this implies

$$
\nabla^{\prime} f_{m_{0}-1}\left(0^{\prime}\right)=0^{\prime} .
$$

If $f_{m_{0}-1}\left(0^{\prime}\right)=0$ we suppose the coordinate system has been oriented so that (48) holds. These functions divide $\Omega$ into $l+1$ regions

$$
\tilde{D}_{m}:=\left\{x \in \Omega \mid f_{m-1}\left(x^{\prime}\right)<x_{n}<f_{m}\left(x^{\prime}\right)\right\}, \quad 1 \leqq m \leqq l+1,
$$

where we have adopted the notation $f_{0} \equiv-1, f_{l+1} \equiv 1$. In an appropriate sense one may think of $\tilde{D}_{m}$ as $D_{m} \cap \Omega$. Let $A^{(m)} \in C^{\mu}\left(\overline{\tilde{D}_{m}}\right), 1 \leqq m \leqq l+1$, be symmetric, positive definite matrix-valued functions, and define

$$
A(x)=A^{(m)}(x), \quad x \in \tilde{D}_{m}, 1 \leqq m \leqq l+1 .
$$


Let $g^{(m)}=\left(g_{1}^{(m)}, \ldots, g_{n}^{(m)}\right)$ be in $C^{\mu}\left(\overline{\tilde{D}_{m}}\right)$, and define

$$
g(x)=g^{(m)}(x), \quad x \in \tilde{D}_{m}, 1 \leqq m \leqq l+1 .
$$

We introduce the $l+1$ "strips"

$$
\Omega_{m}=\left\{x \in \Omega \mid f_{m-1}\left(0^{\prime}\right)<x_{n}<f_{m}\left(0^{\prime}\right)\right\},
$$

and define the piecewise constant (matrix-valued) function

$$
\bar{A}(x)=\left\{\begin{array}{lll}
A^{(m)}\left(0^{\prime}, f_{m-1}\left(0^{\prime}\right)\right), & x \in \Omega_{m}, & m>m_{0}, \\
A^{\left(m_{0}\right)}(0), & x \in \Omega_{m_{0}}, & \\
A^{(m)}\left(0^{\prime}, f_{m}\left(0^{\prime}\right)\right), & x \in \Omega_{m}, & m<m_{0} .
\end{array}\right.
$$

Using $g^{(m)}, 1 \leqq m \leqq l+1$ we similarly define a piecewise constant vector-valued function $\bar{G}$. Since $f_{m} \in C^{1, \alpha}\left(\left|x^{\prime}\right| \leqq 1\right)$, we have

$$
f_{m}\left(x^{\prime}\right)=f_{m}\left(0^{\prime}\right)+\nabla f_{m}\left(0^{\prime}\right) x^{\prime}+O\left(\left|x^{\prime}\right|^{1+\alpha}\right),
$$

where $\left|O\left(\left|x^{\prime}\right|^{1+\alpha}\right)\right| \leqq C\left|x^{\prime}\right|^{1+\alpha}$ for some $C$ depending only on the $C^{1, \alpha}$ norm of $f_{m}$. We also know that $f_{m}\left(x^{\prime}\right)>f_{m-1}\left(x^{\prime}\right)$ for $\left|x^{\prime}\right| \leqq 1$. It follows that

$$
\left|\nabla f_{m}\left(0^{\prime}\right)-\nabla f_{m-1}\left(0^{\prime}\right)\right| \leqq C\left(f_{m}\left(0^{\prime}\right)-f_{m-1}\left(0^{\prime}\right)\right)^{\frac{\alpha}{\alpha+1}}
$$

By a "telescoping summation" argument we now, in view of (46), (47) and (48), conclude that

$$
\left|\nabla f_{m}\left(0^{\prime}\right)\right| \leqq C\left|f_{m}\left(0^{\prime}\right)\right|^{\frac{\alpha}{\alpha+1}}
$$

The constant $C$ now depends on $l$ and the $C^{1, \alpha}$ norm of $f_{m}$.

From the definition of the "strip" $\Omega_{m}$ one would expect that, generically, a significant fraction of $\tilde{D}_{m}$, locally, falls inside $\Omega_{m}$. The following lemma makes this statement more precise by estimating the smallness of $\left(\tilde{D}_{m} \cap s \Omega\right) \backslash \Omega_{m}$, for $0<s \leqq 1$.

Lemma 5.1. There exists a constant $C$, depending on $l$ and the $C^{1, \alpha}$ norm of $f_{m}$, $1 \leqq m \leqq l$, such that

$$
s^{-n}\left|\left(\tilde{D}_{m} \cap s \Omega\right) \backslash \Omega_{m}\right| \leqq C s^{\frac{\alpha}{\alpha+1}}, \quad 1 \leqq m \leqq l+1 .
$$

Proof. Due to the identity $\left(\tilde{D}_{m} \cap s \Omega\right) \backslash \Omega_{m}=\cup_{j \neq m} \tilde{D}_{m} \cap s \Omega \cap \Omega_{j}$, it suffices to prove that

$$
\left|\tilde{D}_{m} \cap s \Omega \cap \Omega_{j}\right| \leqq C s^{\frac{\alpha}{\alpha+1}}, \quad j \neq m .
$$


If the set $\tilde{D}_{m} \cap s \Omega \cap \Omega_{j}=\emptyset$, there is nothing to prove. We thus assume it is nonempty. It is quite easy to see that

$$
\begin{aligned}
& \left|\tilde{D}_{m} \cap s \Omega \cap \Omega_{j}\right| \\
& \leqq C\left\{\begin{array}{l}
\min \left\{\int_{(-s, s)^{n-1}}\left|f_{m}\left(x^{\prime}\right)-f_{m}\left(0^{\prime}\right)\right|, \int_{(-s, s)^{n-1}}\left|f_{j-1}\left(x^{\prime}\right)-f_{j-1}\left(0^{\prime}\right)\right|\right\} \\
\quad \text { for } j>m, \\
\min \left\{\int_{(-s, s)^{n-1}}\left|f_{j}\left(x^{\prime}\right)-f_{j}\left(0^{\prime}\right)\right|, \int_{(-s, s)^{n-1}}\left|f_{m-1}\left(x^{\prime}\right)-f_{m-1}\left(0^{\prime}\right)\right|\right\} \\
\quad \text { for } j<m .
\end{array}\right.
\end{aligned}
$$

In order to estimate the right-hand side of this inequality it is convenient to introduce the index set

$$
I_{s}=\left\{k \mid\left(x^{\prime}, f_{k}\left(x^{\prime}\right)\right) \in s \Omega \text { for some } x^{\prime}\right\} .
$$

From (49) it follows that

$$
\left|f_{k}\left(0^{\prime}\right)\right| \leqq C s \quad \forall k \in I_{s} .
$$

In combination with (49) and (51) this leads to

$$
\begin{aligned}
s^{-n} \int_{x^{\prime} \in(-s, s)^{n-1}}\left|f_{k}\left(x^{\prime}\right)-f_{k}\left(0^{\prime}\right)\right| d x^{\prime} \\
\quad \leqq C s^{-n} \int_{x^{\prime} \in(-s, s)^{n-1}}\left(\left|f_{k}\left(0^{\prime}\right)\right|^{\frac{\alpha}{\alpha+1}}\left|x^{\prime}\right|+\left|x^{\prime}\right|^{1+\alpha}\right) d x^{\prime} \\
\quad \leqq C\left(s^{\frac{\alpha}{\alpha+1}}+s^{\alpha}\right) \leqq C s^{\frac{\alpha}{\alpha+1}} \quad \forall k \in I_{s} .
\end{aligned}
$$

It is also easy to see that

$$
\begin{aligned}
& k>m_{0}, \quad \tilde{D}_{k} \cap s \Omega \neq \emptyset \Rightarrow m_{0}, \cdots, k-1 \in I_{s}, \\
& k<m_{0}, \quad \tilde{D}_{k} \cap s \Omega \neq \emptyset \Rightarrow k, \cdots, m_{0}-1 \in I_{s} .
\end{aligned}
$$

At this point we divide our proof into three different cases.

Case $1 . m>m_{0}$. Since $\tilde{D}_{m} \cap s \Omega \neq \emptyset$, it follows from the first statement of (56) that $m-1$ is in $I_{s}$. A combination of (53) and (55) (with $k=m-1$ ) gives the estimate (52) for $j<m$. On the other hand, for $j>m>m_{0}$, the fact that $s \Omega \cap \Omega_{j} \neq \varnothing$ immediately implies that $\left(0^{\prime}, f_{j-1}\left(0^{\prime}\right)\right) \in s \Omega$. We therefore have $j-1 \in I_{s}$ and the estimate (52) again follows from a combination of (53) and (55) (with $k=j-1$ ).

Case 2. $m<m_{0}$. Since $\tilde{D}_{m} \cap s \Omega \neq \emptyset$, it follows from the second statement of (56) that $m$ is in $I_{s}$. A combination of (53) and (55) (with $k=m$ ) gives the estimate (52) for $j>m$. On the other hand, for $j<m<m_{0}$, the fact that $s \Omega \cap \Omega_{j} \neq \varnothing$ immediately implies that $\left(0^{\prime}, f_{j}\left(0^{\prime}\right)\right) \in s \Omega$. We therefore have $j \in I_{s}$ and the estimate (52) again follows from a combination of (53) and (55) (with $k=j$ ).

Case 3. $m=m_{0}$. Here we conclude from $s \Omega \cap \Omega_{j} \neq \emptyset$ that

$$
\begin{array}{rlrl}
m_{0} & \in I_{S} & & \text { for } j>m_{0}, \\
m_{0}-1 \in I_{S} & \text { for } j<m_{0} .
\end{array}
$$

As before, the estimate follows from a combination of (53) and (55). 
Using the previous lemma, we may now quite easily establish the equivalent of Lemma 4.3.

Lemma 5.2. Suppose $q>n$ and $0<\alpha^{\prime} \leqq \min \left\{\mu, \frac{\alpha}{(\alpha+1) q}\right\}$. Let $A$ and $\bar{A}$ be as introduced at the beginning of this section, and let $A_{r_{0}}$ and $\bar{A}_{r_{0}}$ be defined by $A_{r_{0}}(x)=A\left(r_{0} x\right), \bar{A}_{r_{0}}(x)=\bar{A}\left(r_{0} x\right)$. Given any $\varepsilon_{0}>0$ there exists a positive constant $r_{0}$, depending on $n, q, l, \varepsilon_{0}, \alpha, \alpha^{\prime}, \bar{\lambda}, \bar{\Lambda}, \max _{1 \leqq m \leqq l}\left\|f_{m}\right\|_{C^{1, \alpha}\left([-1,1]^{n-1}\right)}$, and $\max _{1 \leqq m \leqq l+1}\left\|A^{(m)}\right\|_{C^{\alpha^{\prime}}\left(\tilde{D}_{m}\right)}$, so that

$$
\left(f_{r \Omega}\left|A_{r_{0}}(x)-\bar{A}_{r_{0}}(x)\right|^{q}\right)^{1 / q} \leqq \varepsilon_{0} r^{\alpha^{\prime}}, \quad \forall 0<r \leqq 1 .
$$

Proof. For $0<r \leqq 1$, a change of variable gives

$$
\left(f_{r \Omega}\left|A_{r_{0}}(x)-\bar{A}_{r_{0}}(x)\right|^{q} d x\right)^{1 / q}=\left(f_{s \Omega}|A(x)-\bar{A}(x)|^{q} d x\right)^{1 / q}
$$

with $s=r_{0} r \in\left(0, r_{0}\right)$. Due to the definition of $A, \tilde{D}_{m}$, and $\Omega_{m}$, and due to Lemma 5.1

$$
\begin{aligned}
& \left(f_{s \Omega}|A(x)-\bar{A}(x)|^{q} d x\right)^{1 / q} \\
& =\left(s^{-n} \sum_{m} \int_{\tilde{D}_{m} \cap s \Omega \cap \Omega_{m}}\left|A^{(m)}(x)-\bar{A}(x)\right|^{q} d x\right. \\
& \left.\quad+s^{-n} \sum_{m} \int_{\left(\tilde{D}_{m} \cap s \Omega\right) \backslash \Omega_{m}}\left|A^{(m)}(x)-\bar{A}(x)\right|^{q} d x\right)^{1 / q} \\
& \leq\left(s^{-n} \sum_{m} \int_{\tilde{D}_{m} \cap s \Omega \cap \Omega_{m}}\left|A^{(m)}(x)-\bar{A}(x)\right|^{q} d x\right)^{1 / q}+C s^{\frac{\alpha}{(\alpha+1) q}} .
\end{aligned}
$$

The first term in the right-hand side of (58) requires a slightly different estimation, depending on whether $m<m_{0}, m=m_{0}$ or $m>m_{0}$.

For $m<m_{0}$,

$$
\begin{aligned}
\left(s^{-n}\right. & \left.\int_{\tilde{D}_{m} \cap s \Omega \cap \Omega_{m}}\left|A^{(m)}(x)-\bar{A}(x)\right|^{q} d x\right)^{1 / q} \\
& =\left(s^{-n} \int_{\tilde{D}_{m} \cap s \Omega \cap \Omega_{m}}\left|A^{(m)}(x)-A^{(m)}\left(0^{\prime}, f_{m}\left(0^{\prime}\right)\right)\right|^{q} d x\right)^{1 / q} \\
& \leqq C\left(s^{-n} \int_{\tilde{D}_{m} \cap s \Omega \cap \Omega_{m}}\left|x-\left(0^{\prime}, f_{m}\left(0^{\prime}\right)\right)\right|^{\alpha^{\prime} q} d x\right)^{1 / q} \leqq C s^{\alpha^{\prime}}
\end{aligned}
$$


For $m=m_{0}$,

$$
\begin{aligned}
& \left(s^{-n} \int_{\tilde{D}_{m_{0}} \cap s \Omega \cap \Omega_{m_{0}}}\left|A^{(m)}(x)-\bar{A}(x)\right|^{q} d x\right)^{1 / q} \\
& \quad=\left(s^{-n} \int_{\tilde{D}_{m_{0}} \cap s \Omega \cap \Omega_{m_{0}}}\left|A^{\left(m_{0}\right)}(x)-A^{\left(m_{0}\right)}(0)\right|^{q} d x\right)^{1 / q} \leqq C s^{\alpha^{\prime}} .
\end{aligned}
$$

For $m>m_{0}$,

$$
\begin{aligned}
\left(s^{-n}\right. & \left.\int_{\tilde{D}_{m} \cap s \Omega \cap \Omega_{m}}\left|A^{(m)}(x)-\bar{A}(x)\right|^{q} d x\right)^{1 / q} \\
& =\left(s^{-n} \int_{\tilde{D}_{m} \cap s \Omega \cap \Omega_{m}}\left|A^{(m)}(x)-A^{(m)}\left(0^{\prime}, f_{m-1}\left(0^{\prime}\right)\right)\right|^{q} d x\right)^{1 / q} \\
& \leqq C\left(s^{-n} \int_{\tilde{D}_{m} \cap s \Omega \cap \Omega_{m}}\left|x-\left(0^{\prime}, f_{m-1}\left(0^{\prime}\right)\right)\right|^{\alpha^{\prime} q} d x\right)^{1 / q} \leqq C s^{\alpha^{\prime}} .
\end{aligned}
$$

In either case we therefore from (58) conclude that

$$
\begin{aligned}
\left(f_{s \Omega}|A(x)-\bar{A}(x)|^{q} d x\right)^{1 / q} & \leqq C\left(s^{\alpha^{\prime}}+s^{\frac{\alpha}{(\alpha+1) q}}\right) \\
& \leqq C s^{\alpha^{\prime}}=C r_{0}^{\alpha^{\prime}} r^{\alpha^{\prime}}
\end{aligned}
$$

We now simply choose $r_{0}$, so that $C r_{0}^{\alpha^{\prime}}=\varepsilon_{0}$, and the lemma follows.

We may combine Proposition 3.2 with Lemma 5.2 to establish the following proposition.

Proposition 5.3. Let $A \in \mathcal{A}(\bar{\lambda}, \bar{\Lambda}), \bar{A} \in \overline{\mathcal{A}}(\bar{\lambda}, \bar{\Lambda})$. Let $h \in L^{\infty}(\Omega)$, and let $g$ and $\bar{G}$ be as defined at the beginning of this section. For any $q>n$, and any $0<\alpha^{\prime} \leqq \min \left\{\mu, \frac{\alpha}{(\alpha+1) q}\right\}$ there exist constants $C$ and $r_{0}$ such that, if $u \in H^{1}(\Omega)$ is a solution to

$$
\partial_{i}\left(A_{i j} \partial_{j} u\right)=h+\partial_{i} g_{i} \quad \text { in } \Omega,
$$

with

$$
\|u\|_{L^{\infty}(\Omega)}+\|h\|_{L^{\infty}(\Omega)}+\max _{1 \leqq m \leqq l+1}\left\|g^{(m)}\right\|_{C^{\alpha^{\prime}}\left(\overline{\tilde{D}_{m}}\right)} \leqq 1,
$$

then one may find a continuous, piecewise linear function, $p$, whose coefficients are bounded in absolute value by $C$, and which satisfies

$$
\partial_{i}\left(\bar{A}_{i j} \partial_{j} p(x)\right)=\partial_{i} \bar{G}_{i}, \quad \text { in } r_{0} \Omega .
$$

and

$$
|u(x)-p(x)| \leqq C|x|^{1+\alpha^{\prime}}, \quad x \in r_{0} \Omega,
$$

The constants $C$ and $r_{0}$ depend on $n, \alpha^{\prime}, \alpha, q, \bar{\lambda}, \bar{\Lambda}$, the number $l, \max _{1 \leqq m \leqq l}$ $\left\|A^{(m)}\right\|_{C^{\alpha^{\prime}}\left(\overline{\tilde{D}_{m}}\right)}$, and $\max _{1 \leqq m \leqq l}\left\|f_{m}\right\|_{C^{1, \alpha}\left([-1,1]^{n-1}\right)}$. 
Proof. We consider $w(x)=u\left(r_{0} x\right)$, that solves the equation

$$
\partial_{i}\left(\left(A_{r_{0}}\right)_{i j} \partial_{j} w\right)=r_{0}^{2} h\left(r_{0} \cdot\right)+r_{0} \partial_{i} g_{i}\left(r_{0} \cdot\right) \text { in } \Omega .
$$

By selecting $r_{0}$ as small as prescribed in Lemma 5.2, we obtain the estimate

$$
\left\|A_{r_{0}}-\bar{A}_{r_{0}}\right\|_{Y^{1+\alpha^{\prime}, q}} \leqq \varepsilon_{0},
$$

exactly as required by the crucial hypothesis (32) in Proposition 3.2. Applying Lemma 5.2 to the functions $g$ and $\bar{G}$ we may select $r_{0}$ sufficiently small that

$$
\left\|g\left(r_{0} \cdot\right)-\bar{G}\left(r_{0} \cdot\right)\right\|_{Y^{1+\alpha^{\prime}, q}} \leqq \frac{1}{2} \varepsilon_{0} .
$$

By selecting $r_{0}$ sufficiently small we thus get

$$
\begin{gathered}
r_{0}^{2}\left\|h\left(r_{0} \cdot\right)\right\|_{Y^{\alpha^{\prime}, q / 2}}+r_{0}\left\|g\left(r_{0} \cdot\right)-\bar{G}\left(r_{0} \cdot\right)\right\|_{Y^{1+\alpha^{\prime}, q}} \\
\leqq r_{0}^{2}\|h\|_{L^{\infty}(\Omega)}+r_{0} \frac{1}{2} \varepsilon_{0} \leqq \varepsilon_{0} .
\end{gathered}
$$

We also have

$$
r_{0}\left\|\bar{G}\left(r_{0} \cdot\right)\right\|_{L^{\infty}(\Omega)} \leqq r_{0} \max _{1 \leqq m \leqq l+1}\left\|g^{(m)}\right\|_{C^{\alpha^{\prime}}\left(\tilde{D}_{m}\right)} \leqq 1
$$

We remind the reader that the ellipticity bounds for $A_{r_{0}}$ and $\bar{A}_{r_{0}}$ are the same as those for $A$ and $\bar{A}$. We also note that any estimates obtained for $w$ will translate into similar estimates for $u$ except for a fixed constant depending on $r_{0}$.

Since the hypotheses are satisfied we may now apply Proposition 3.2 (with $\bar{H}=0$ ) to $u$. This leads to the existence of a continuous, piecewise linear polynomial $q$, whose coefficients are bounded in absolute value by $C$, and which satisfies

$$
\partial_{i}\left(\bar{A}_{i j}\left(r_{0} x\right) \partial_{j} q(x)\right)=r_{0} \partial_{i} \bar{G}_{i}\left(r_{0} x\right), \quad \text { in } \frac{1}{4} \Omega .
$$

and

$$
|w(x)-q(x)| \leqq C|x|^{1+\alpha^{\prime}}, \quad x \in \frac{1}{4} \Omega,
$$

The constant $\mathrm{C}$ depends on $n, \alpha^{\prime}, \alpha, q, \bar{\lambda}, \bar{\Lambda}$, the number $l, \max _{1 \leqq m \leqq l+1}$ $\left\|A^{(m)}\right\|_{C^{\alpha^{\prime}}\left(\tilde{D}_{m}\right)}$, and $\max _{1 \leqq m \leqq l}\left\|f_{m}\right\|_{C^{1, \alpha}\left([-1,1]^{n-1}\right)}$. The function $p(x)=q\left(x / r_{0}\right)$ satisfies all the requirements from the statement of this proposition (after $\frac{1}{4} r_{0}$ is renamed $r_{0}$ ).

Proof of Theorem 1.1. Using the same arguments as those leading to Theorem 4.1 we conclude from Proposition 5.3 that

$$
\|\nabla u\|_{L^{\infty}\left(D_{\varepsilon}\right)} \leqq C\left(\|u\|_{L^{\infty}(D)}+\|h\|_{L^{\infty}(D)}+\max _{1 \leqq m \leqq L}\left\|g^{(m)}\right\|_{C^{\alpha^{\prime}\left(\bar{D}_{m}\right)}}\right) .
$$

Here we note that $l$ (the number of curves) that appears in our local estimates is bounded by a constant that depends on the $C^{1, \alpha}$ modulus of $\cup_{m=1}^{L} \partial D_{m}$. To complete the proof of Theorem 1.1 we only need to establish

$$
|\nabla u(x)-\nabla u(0)| \leqq C|x|^{\alpha^{\prime}} \quad \forall x \in \tilde{D}_{m_{0}}
$$


where $u$ is as in Proposition 5.3. Naturally, we only need to establish this estimate for $|x|<r_{1}$ for some small $r_{1}$, which depends on the same parameters as the constant $C$ in Theorem 1.1. In the following we repeatedly use the smallness of $x$ (i.e., $\left.r_{1}\right)$ without explicit mention. For any $x \in \tilde{D}_{m_{0}} \backslash\{0\}$, if $f_{m_{0}}\left(0^{\prime}\right)>80|x|$, set $\bar{x}=\left(0^{\prime}, 10|x|\right)$ (and $\left.m=m_{0}-1\right)$, otherwise let $m \geqq m_{0}$ be the smallest index for which $f_{m+1}\left(0^{\prime}\right)-f_{m}\left(0^{\prime}\right)>80|x|$ and set $\bar{x}=\left(0^{\prime}, f_{m}\left(0^{\prime}\right)+10|x|\right)$. Clearly, $10|x| \leqq|\bar{x}| \leqq 100(l+1)|x|$. We will first show that

$$
|\nabla u(\bar{x})-\nabla p(\bar{x})| \leqq C|x|^{\alpha^{\prime}},
$$

where $p$ is the piecewise linear function from Proposition 5.3. This in turn implies

$$
\left|\nabla u(\bar{x})-T^{(m+1)} \cdots T^{\left(m_{0}+1\right)} \nabla u(0)\right| \leqq C|x|^{\alpha^{\prime}},
$$

where $T^{(j)} b=M^{(j)} b+\left(0, \frac{1}{\bar{A}_{n n}^{(j)}}\left[\bar{G}_{n}^{(j)}-\bar{G}_{n}^{(j-1)}\right]\right)$, and $M^{(j)}$ is the transmission matrix corresponding to the horizontal hyperplane $x_{n}=f_{j-1}\left(0^{\prime}\right)$. For $j>m_{0}+$ $1, T^{(j)}$ is determined by the values of $A^{(j)}\left(0^{\prime}, f_{j-1}\left(0^{\prime}\right)\right), A^{(j-1)}\left(0^{\prime}, f_{j-2}\left(0^{\prime}\right)\right)$, $g^{(j)}\left(0^{\prime}, f_{j-1}\left(0^{\prime}\right)\right), g^{(j-1)}\left(0^{\prime}, f_{j-2}\left(0^{\prime}\right)\right)$, while $T^{\left(m_{0}+1\right)}$ is determined by the values of $\left.A^{\left(m_{0}+1\right)}\left(0^{\prime}, f_{m_{0}}\left(0^{\prime}\right)\right), A^{\left(m_{0}\right)}(0)\right), g^{\left(m_{0}+1\right)}\left(0^{\prime}, f_{m_{0}}\left(0^{\prime}\right)\right)$, and $g^{\left(m_{0}\right)}(0)$.

From the definition of $\bar{x}$ and $m$, and the smallness of $|x|$ it follows immediately that the neighborhood

$$
\hat{D}=\bar{x}+4(-|x|,|x|)^{n}=\left(0^{\prime},|\bar{x}|\right)+4(-|x|,|x|)^{n},
$$

lies inside $\tilde{D}_{m+1}$ (and $\left.\Omega_{m+1}\right)$. Define the function

$$
\hat{w}(y)=\frac{u(\bar{x}+4|x| y)-p(\bar{x}+4|x| y)}{(4|x|)^{1+\alpha^{\prime}}}, \quad y \in \Omega .
$$

In view of Proposition 5.3, $\hat{w}(y)$ satisfies

$$
\|\hat{w}\|_{L^{\infty}(\Omega)} \leqq C .
$$

At the same time, since $\bar{x}+4|x| y \in \tilde{D}_{m+1} \cap \Omega_{m+1}$,

$$
\partial_{i}\left(A_{i j}^{(m+1)}(\bar{x}+4|x| y) \partial_{j}\right) \hat{w}(y)=\hat{h}(y)+\partial_{i} \hat{g}_{i}(y),
$$

where

$$
\hat{h}(y)=(4|x|)^{1-\alpha^{\prime}} h(\bar{x}+4|x| y),
$$

and

$$
\begin{aligned}
& \hat{g}_{i}(y)=(4|x|)^{-\alpha^{\prime}}\left[g_{i}^{(m+1)}(\bar{x}+4|x| y)-g_{i}^{(m+1)}\left(0^{\prime}, f_{m}\left(0^{\prime}\right)\right)\right] \\
& \quad-(4|x|)^{-1-\alpha^{\prime}}\left[A_{i j}^{(m+1)}(\bar{x}+4|x| y)-A_{i j}^{(m+1)}\left(0^{\prime}, f_{m}\left(0^{\prime}\right)\right)\right] \partial_{j} p(\bar{x}+4|x| y) .
\end{aligned}
$$

The functions $g^{(m+1)}$ and $A^{(m+1)}$ are Hölder continuous (with exponent $\mu \geqq \alpha^{\prime}$ ) so it is easy to see that

$$
\|\hat{h}\|_{L^{\infty}(\Omega)} \leqq C, \quad\left\|\hat{g}_{i}\right\|_{C^{\mu}(\Omega)} \leqq C .
$$


Since $\left\|A^{(m+1)}(\bar{x}+4|x| \cdot)\right\|_{C^{\mu}(\Omega)} \leqq C$, we can now apply Schauder theory to obtain

$$
|\nabla \hat{w}(y)| \leqq C, \quad y \in \frac{2}{3} \Omega .
$$

Insertion of $y=0$ yields

$$
|x|^{-\alpha^{\prime}}|\nabla u(\bar{x})-\nabla p(\bar{x})| \leqq C,
$$

which immediately implies (63).

Now let $z$ be on either the graph of $f_{m_{0}}$ or $f_{m_{0}-1}$, so that $|z-x|_{2}$ is the minimal (Euclidean) distance of $x \in \tilde{D}_{m_{0}} \backslash\{0\}$ to the union of the graphs of $\left\{f_{j}\right\}$. We may without loss of generality assume that $z$ lies on the graph of $f_{m_{0}-1}$. Let $L$ be the line passing through $z$ which is normal to this graph. Clearly $x \in L$. Let $z^{(j)}$ denote the intersection of $L$ with the graph of $f_{j}$ for $m_{0}-1 \leqq j \leqq m+1$. It is not difficult to see that (due to the smallness of $|x|$ )

$$
\left|z^{(j)}-\left(0^{\prime}, f_{j}\left(0^{\prime}\right)\right)\right| \leqq 4|x|, \quad m_{0} \leqq j \leqq m
$$

and

$$
\left|z^{(m+1)}-z^{(m)}\right| \geqq 40|x| .
$$

Here $m$ is as defined before, and we have used the fact that the point $\left(0^{\prime}, f_{m_{0}-1}\left(0^{\prime}\right)\right)$ is the projection of the origin onto the graph of the function $f_{m_{0}-1}$. A slight change of our earlier argument shows that (when $m \geqq m_{0}$ ) we can find $\bar{z}$ on the segment determined by $z^{(m)}$ and $z^{(m+1)}$ with $\left|\bar{z}-z^{(m)}\right|=10|x|$ such that

$$
\left|\nabla u(\bar{z})-\widetilde{T}^{(m+1)} \cdots \widetilde{T}^{\left(m_{0}+1\right)} \nabla u(x)\right| \leqq C|x|^{\alpha^{\prime}},
$$

where for $j \geqq m_{0}+1, \widetilde{T}^{(j)}$ describes the transition at the hyperplane orthogonal to $L$ and passing through $z^{(j-1)} ; \widetilde{T}^{(j)}, j>m_{0}+1$, is determined from the values of $A^{(j)}\left(z^{j-1}\right), A^{(j-1)}\left(z^{j-2}\right), g^{(j)}\left(z^{j-1}\right), g^{(j-1)}\left(z^{j-2}\right)$, while $\widetilde{T}^{\left(m_{0}+1\right)}$ is determined from the values of $A^{\left(m_{0}+1\right)}\left(z^{\left(m_{0}\right)}\right), A^{\left(m_{0}\right)}(x), g^{\left(m_{0}+1\right)}\left(z^{\left(m_{0}\right)}\right)$, and $g^{\left(m_{0}\right)}(x)$. When $m=m_{0}-1$ a slight change of our previous argument yields a point $\bar{z}$ with $|\bar{z}-x|=10|x|$ such that (68) holds (in this case $\widetilde{T}^{(m+1)} \ldots \widetilde{T}^{\left(m_{0}+1\right)}=I$ ). Due to (66) and the Hölder continuity of $A^{(j)}$ and $g^{(j)}$, we have

$$
\left|T^{(j)}-\widetilde{T}^{(j)}\right| \leqq C|x|^{\mu}
$$

So,

$$
\left|\nabla u(\bar{z})-T^{(m+1)} \cdots T^{\left(m_{0}+1\right)} \nabla u(x)\right| \leqq C|x|^{\alpha^{\prime}} .
$$

It is easy to see that we may suppose

$$
|\bar{x}-\bar{z}| \leqq 2|x|
$$

We may now insert $y=(\bar{z}-\bar{x}) / 4|x|$ into the estimate (65) to obtain

$$
|\nabla u(\bar{z})-\nabla p(\bar{z})| \leqq C|x|^{\alpha^{\prime}} .
$$


Since $\bar{x}$ and $\bar{z}$ both lie in $\Omega_{m+1}$ we have $\nabla p(\bar{z})=\nabla p(\bar{x})=T^{(m+1)} \cdots T^{\left(m_{0}+1\right)}$ $\nabla u(0)$, and so the above estimate becomes

$$
\left|\nabla u(\bar{z})-T^{(m+1)} \cdots T^{\left(m_{0}+1\right)} \nabla u(0)\right| \leqq C|x|^{\alpha^{\prime}} .
$$

In terms of the matrices $N^{(m)}$, introduced in the proof of Corollary 2.6, a combination of (69), and (70) immediately yields

$$
\begin{aligned}
& \left|\left(N^{(m+1)}\right)^{-1} N^{\left(m_{0}\right)}[\nabla u(x)-\nabla u(0)]\right| \\
& \quad=\left|T^{(m+1)} \cdots T^{\left(m_{0}+1\right)} \nabla u(x)-T^{(m+1)} \cdots T^{\left(m_{0}+1\right)} \nabla u(0)\right| \\
& \quad \leqq C|x|^{\alpha^{\prime}},
\end{aligned}
$$

which leads to

$$
|\nabla u(x)-\nabla u(0)| \leqq C|x|^{\alpha^{\prime}} .
$$

This completes the proof of Theorem 1.1.

\section{Boundary estimates}

Theorem 1.2 is established using a string of lemmas similar to those used in the proof of Theorem 1.1. We start with the local estimates, in which case the domain $\Omega$ is replaced by

$$
\Omega^{+}=(-1,1)^{n-1} \times(0,1),
$$

and a boundary condition is imposed on the side $x_{n}=0$. The following notation is quite similar to that used in earlier sections. Let

$$
0 \equiv c_{0}<c_{1}<\cdots<c_{l+1} \equiv 1,
$$

and define

$$
\Omega_{m}=\left\{x \in \Omega^{+} \mid c_{m-1}<x_{n}<c_{m}\right\}, \quad 1 \leqq m \leqq l+1 .
$$

Let $\left\{\bar{A}^{(m)}\right\}_{1}^{l+1}=\left\{\left(\bar{A}_{i j}^{(m)}\right)\right\}_{1}^{l+1}$ be $l+1$ symmetric, positive definite (constant) matrices, with $0<\bar{\lambda} \leqq \bar{A}^{(m)} \leqq \bar{\Lambda}<\infty$, and define a matrix-valued function $\bar{A}(x)=\left(\bar{A}_{i j}(x)\right)$ by

$$
\bar{A}_{i j}(x)=\bar{A}_{i j}^{(m)}, \quad x \in \Omega_{m}, \quad 1 \leqq m \leqq l+1, .
$$

By a slight extension of our previous notation $\bar{A} \in \overline{\mathcal{A}}(\bar{\lambda}, \bar{\Lambda})$. Let $\left\{\bar{H}^{(m)}\right\}_{1}^{l+1}$ be $l+1$ constants and let $\left\{\bar{G}^{(m)}\right\}_{1}^{l+1}$ be $l+1$ vectors in $\mathbb{R}^{n}$. We define the functions $\bar{H}(x)$ and $\bar{G}(x)$ by

$$
\bar{H}(x)=\bar{H}^{(m)}, \quad \bar{G}(x)=\bar{G}^{(m)}, \quad x \in \Omega_{m}, \quad 1 \leqq m \leqq l+1 .
$$

Finally we require that the boundary value, $\varphi$, satisfies

$$
\varphi \in C^{1, \alpha}\left(\left|x^{\prime}\right| \leqq 1\right) .
$$

The following is an analogue of Proposition 2.1. 
Proposition 6.1. Let $\bar{A}, \bar{G}$ and $\bar{H}$ be as above and let d be a vectord $=\left(d_{0}, d_{1}, \ldots\right.$, $\left.d_{n-1}\right)$. Suppose $k$ is a non-negative integer and suppose $\varepsilon>0$. There exists a constant $C=C(\varepsilon, k, n, \bar{\lambda}, \bar{\Lambda})$, such that if $v \in H^{1}\left(\Omega^{+}\right)$is a solution to

$$
\begin{aligned}
\partial_{i}\left(\bar{A}_{i j}(x) \partial_{j}\right) v & =\bar{H}+\partial_{i} \bar{G}_{i} & & \text { in } \Omega^{+}, \\
v & =d_{0}+\sum_{1 \leqq \beta \leqq n-1} d_{\beta} x_{\beta} & & \text { on }\left\{\left|x^{\prime}\right|<1, x_{n}=0\right\},
\end{aligned}
$$

then

$$
\begin{aligned}
& \max _{1 \leqq m \leqq l+1}\|v\|_{C^{k}\left(\bar{\Omega}_{m} \cap(1-\varepsilon) \Omega^{+}\right)} \\
& \quad \leqq C\left(\|v\|_{L^{\infty}\left(\Omega^{+}\right)}+\|\bar{H}\|_{L^{\infty}\left(\Omega^{+}\right)}+\|\bar{G}\|_{L^{\infty}\left(\Omega^{+}\right)}+|d|\right) .
\end{aligned}
$$

Proof. When $d=0$, the estimate follows from application of the same technique as used for the proof of Proposition 2.1. When $d \neq 0$, the matching condition (22), with $\bar{G}=0$, and the continuity condition (23) determine a unique continuous, piecewise linear function

$$
\hat{p}(x)= \begin{cases}a^{(m)}+b^{(m)} \cdot x, & \text { in } \Omega_{m}, \quad m \geqq 1, \\ d_{0}+\sum_{1 \leqq \beta \leqq n-1} d_{\beta} x_{\beta}, & \text { in } \Omega_{1},\end{cases}
$$

which satisfies

$$
\partial_{i}\left(\bar{A}_{i j}(x) \partial_{j}\right) \hat{p}(x)=0 \quad \text { in } \Omega^{+} .
$$

The coefficients of $\hat{p}$ are bounded by $C|d|$. Applying the $d=0$ case to $v-\hat{p}(x)$, we complete the proof.

Proposition 6.1 leads to an approximation result analogous to Corollary 2.6.

Corollary 6.2. Let $\bar{A}, \bar{G}, \bar{H}$ and $d$ be as in Proposition 6.1, and let $v \in H^{1}\left(\Omega^{+}\right)$ denote a solution to

$$
\begin{aligned}
\partial_{i}\left(\bar{A}_{i j}(x) \partial_{j}\right) v & =\bar{H}+\partial_{i}\left(\bar{G}_{i}\right) & & \text { in } \Omega^{+}, \\
v & =d_{0}+\sum_{1 \leqq \beta \leqq n-1} d_{\beta} x_{\beta} & & \text { on }\left\{\left|x^{\prime}\right|<1, x_{n}=0\right\},
\end{aligned}
$$

with

$$
\|v\|_{L^{\infty}\left(\Omega^{+}\right)}+\|\bar{H}\|_{L^{\infty}\left(\Omega^{+}\right)}+\|\bar{G}\|_{L^{\infty}\left(\Omega^{+}\right)}+|d| \leqq 1 .
$$

There exists a constant $C=C(n, \bar{\lambda}, \bar{\Lambda})$ and a continuous, piecewise linear function

$$
p(x)=a^{(m)}+b^{(m)} \cdot x, \quad x \in \Omega_{m}, \quad 1 \leqq m \leqq l+1,
$$


satisfying

$$
\begin{aligned}
\partial_{i}\left(\bar{A}_{i j}(x) \partial_{j}\right) p(x) & =\partial_{i}\left(\bar{G}_{i}\right) \quad \text { in } \Omega^{+} \\
p\left(x^{\prime}, 0\right) & =d_{0}+\sum_{1 \leqq \beta \leqq n-1} d_{\beta} x_{\beta},
\end{aligned}
$$

such that

$$
|v(x)-p(x)| \leqq C|x|^{2}, \quad x \in \frac{1}{2} \Omega^{+}
$$

and

$$
\left|a^{(m)}\right|+\left|b^{(m)}\right| \leqq C, \quad 1 \leqq m \leqq m^{*}
$$

Proof. The proof is very similar to that of Corollary 2.6. Only, instead of (21), we set

$$
a^{(1)}=v(0)=d_{0}, \quad b^{(1)}=\nabla v(0)=\left(d_{1}, \ldots, d_{n-1}, \frac{\partial v}{\partial x_{n}}(0)\right),
$$

and define the rest of the $a^{(m)}, b^{(m)}$ using (22) and (23).

The analogue of the first perturbation lemma (Lemma 3.1) reads

Lemma 6.3. Suppose $A \in \mathcal{A}(\bar{\lambda}, \bar{\Lambda})$. Suppose $g=\left(g_{1}, \cdots, g_{n}\right) \in L^{q}\left(\Omega^{+}\right)$and $h \in L^{q / 2}\left(\Omega^{+}\right)$for some $q>n$. Let $u \in H^{1}\left(\Omega^{+}\right)$be a solution to

$$
\partial_{i}\left(A_{i j} \partial_{j} u\right)=h+\partial_{i} g_{i} \quad \text { in } \Omega^{+},
$$

with

$$
u=\varphi \quad \text { on }\left\{\left|x^{\prime}\right|<1, x_{n}=0\right\}
$$

and

$$
\|u\|_{L^{\infty}\left(\Omega^{+}\right)} \leqq 1 .
$$

There exist positive constants $\gamma \leqq 1$ and $C$ (depending only on $q, n, \bar{\lambda}$ and $\bar{\Lambda}$ ) such that if

$$
\left(f_{\Omega^{+}}|A-\bar{A}|^{q} d x\right)^{1 / q} \leqq \varepsilon
$$

for some $\varepsilon>0$, then we may find a function $v \in H^{1}$ with

$$
\begin{aligned}
\partial_{i}\left(\bar{A}_{i j}(x) \partial_{j} v\right) & =\bar{H}+\partial_{i}\left(\bar{G}_{i}\right) & & \text { in } \frac{3}{4} \Omega^{+}, \\
v & =\varphi\left(0^{\prime}\right)+\sum_{1 \leqq \beta \leqq n-1} \partial_{\beta} \varphi\left(0^{\prime}\right) x_{\beta} & & \text { on }\left\{\left|x^{\prime}\right|<3 / 4, x_{n}=0\right\},
\end{aligned}
$$


and with

$$
\begin{aligned}
\|u-v\|_{L^{\infty}\left(\frac{1}{2} \Omega^{+}\right)} & \\
\leqq & C\left(\left[1+\|\bar{G}\|_{L^{\infty}\left(\Omega^{+}\right)}+\|\bar{H}\|_{L^{\infty}\left(\Omega^{+}\right)}+\left|\varphi\left(0^{\prime}\right)\right|+\left|\nabla^{\prime} \varphi\left(0^{\prime}\right)\right|\right] \varepsilon^{\gamma}\right. \\
& +\|g-\bar{G}\|_{L^{q}\left(\Omega^{+}\right)}+\|h-\bar{H}\|_{L^{q / 2}\left(\Omega^{+}\right)}+\| \varphi-\varphi\left(0^{\prime}\right) \\
& \left.\quad-\sum_{1 \leqq \beta \leqq n-1} \partial_{\beta} \varphi\left(0^{\prime}\right) x_{\beta} \|_{L^{\infty}\left(\left|x^{\prime}\right|<1\right)}\right) .
\end{aligned}
$$

Proof. The proof is very similar to that of Lemma 3.1. Of course, in place of Proposition 2.1 we use Proposition 6.1. We just point out how to define $v$, since the rest of the changes are fairly obvious. Let $v$ denote the solution of

$$
\begin{aligned}
\partial_{i}\left(\bar{A}_{i j}(x) \partial_{j} v\right) & =\bar{H}+\partial_{i}\left(\bar{G}_{i}\right) & & \text { in } \frac{3}{4} \Omega^{+}, \\
v & =\varphi\left(0^{\prime}\right)+\sum_{1 \leqq \beta \leqq n-1} \partial_{\beta} \varphi\left(0^{\prime}\right) x_{\beta} & & \text { on }\left\{\left|x^{\prime}\right|<3 / 4, x_{n}=0\right\},
\end{aligned}
$$

and

$$
v=u \quad \text { on } \partial\left(\frac{3}{4} \Omega^{+}\right) \cap\left\{x_{n}>0\right\} .
$$

We (re)introduce the norm

$$
\|h\|_{Y^{s, p}}:=\sup _{0<r<1} r^{1-s}\left(f_{r \Omega^{+}}|h|^{p}\right)^{1 / p},
$$

The previous lemma in combination with Corollary 6.2 leads to the following perturbation result.

Proposition 6.4. Suppose $A \in \mathcal{A}(\bar{\lambda}, \bar{\Lambda})$ and $\bar{A} \in \overline{\mathcal{A}}(\bar{\lambda}, \bar{\Lambda})$, relative to the hyperplanes $x_{n}=c_{m}, 0 \leqq m \leqq l+1$. Suppose $g=\left(g_{1}, \cdots, g_{n}\right) \in L^{q}\left(\Omega^{+}\right), h \in$ $L^{q / 2}\left(\Omega^{+}\right)$for some $\bar{q}>n$, and suppose $\bar{G}=\left(\bar{G}_{1}, \cdots, \bar{G}_{n}\right)$ and $\bar{H}$ are constant on each of the strips $\Omega_{m}$. Let $0<\bar{\alpha}<1$ and let $u \in H^{1}\left(\Omega^{+}\right)$denote a solution to

$$
\begin{aligned}
\partial_{i}\left(A_{i j}(x) \partial_{j} u\right) & =h+\partial_{i} g_{i} & & \text { in } \Omega^{+}, \\
u & =\varphi & & \text { on }\left\{\left|x^{\prime}\right|<1, x_{n}=0\right\},
\end{aligned}
$$

with

$$
\|u\|_{L^{\infty}\left(\Omega^{+}\right)} \leqq 1
$$


There exist constants in the range $0<\sigma<\frac{1}{4}, \varepsilon_{0}>0$, and $C>0$ (depending on $n, q, \bar{\alpha}$, and the ellipticity constants $\bar{\lambda}, \bar{\Lambda}$ ) such that if

$$
\begin{gathered}
\|A-\bar{A}\|_{Y^{1+\bar{\alpha}, q}} \leqq \varepsilon_{0}, \\
\|g-\bar{G}\|_{Y^{1+\bar{\alpha}, q}}+\|h-\bar{H}\|_{Y^{\bar{\alpha}, q / 2}}+\| \varphi-\varphi\left(0^{\prime}\right) \\
-\sum_{1 \leqq \beta \leqq n-1} \partial_{\beta} \varphi\left(0^{\prime}\right) x_{\beta} \|_{C^{1, \bar{\alpha}}\left(\left|x^{\prime}\right|<1\right)} \leqq \varepsilon_{0},
\end{gathered}
$$

and

$$
\|\bar{G}\|_{L^{\infty}(\Omega)}+\|\bar{H}\|_{L^{\infty}(\Omega)}+\|\varphi\|_{C^{1, \bar{\alpha}}\left(\left|x^{\prime}\right| \leqq 1\right)} \leqq 1,
$$

then we may find a sequence of continuous, piecewise linear functions $p_{k}, k=$ $1,2, \ldots$,

$$
p_{k}(x)=a_{k}^{(m)}+b_{k}^{(m)} \cdot x, \quad x \in \Omega_{m}, \quad 1 \leqq m \leqq m^{*},
$$

with

$$
p_{k}\left(x^{\prime}, 0\right)=\varphi\left(0^{\prime}\right)+\sum_{1 \leqq \beta \leqq n-1} \partial_{\beta} \varphi\left(0^{\prime}\right) x_{\beta}
$$

and with

$$
\begin{aligned}
\left|a_{1}^{(m)}\right|+\left|b_{1}^{(m)}\right| & \leqq C \\
\left|a_{k}^{(m)}-a_{k-1}^{(m)}\right| & \leqq C\left[\left(\sigma^{k-1}\right)^{1+\bar{\alpha}}+c_{m-1}\left(\sigma^{k-1}\right)^{\bar{\alpha}}\right], \\
\left|b_{k}^{(m)}-b_{k-1}^{(m)}\right| & \leqq C\left(\sigma^{k-1}\right)^{\bar{\alpha}} \\
b_{k}^{(m)} & =M^{(m)} b_{k}^{(m-1)}+\left(0^{\prime}, \frac{1}{\bar{A}_{n n}^{(m)}}\left[\bar{G}_{n}^{(m)}-\bar{G}_{n}^{(m-1)}\right]\right),
\end{aligned}
$$

for $1 \leqq m \leqq m^{*}$, such that

$$
\left\|u-p_{k}\right\|_{L^{\infty}\left(\sigma^{k} \Omega^{+}\right)} \leqq\left(\sigma^{k}\right)^{1+\bar{\alpha}} .
$$

The limit $p(x)=\lim _{k \rightarrow \infty} p_{k}(x)$ exists for $x \in \frac{1}{4} \Omega^{+}$. It is a continuous, piecewise linear function with coefficients that are uniformly bounded by $C$. Furthermore $p(\cdot)$ satisfies

$$
\begin{aligned}
\partial_{i}\left(\bar{A}_{i j}(x) \partial_{j}\right) p & =\partial_{i}\left(\bar{G}_{i}\right) \quad \text { in } \frac{1}{4} \Omega^{+}, \\
p\left(x^{\prime}, 0\right) & =\varphi\left(0^{\prime}\right)+\sum_{1 \leqq \beta \leqq n-1} \partial_{\beta} \varphi\left(0^{\prime}\right) x_{\beta},
\end{aligned}
$$

and

$$
|u(x)-p(x)| \leqq C|x|^{1+\bar{\alpha}}, \quad x \in \frac{1}{4} \Omega^{+} .
$$


Proof. The proof is a simple modification of that of Proposition 3.2, with Lemma 6.3 substituted for Lemma 3.1.

Before we state the boundary version of Proposition 5.3 we need some additional notation. Let $f_{1}, \cdots, f_{l}$ be $l$ functions in $C^{1, \alpha}\left(\left|x^{\prime}\right| \leqq 1\right)$, satisfying

$$
0<f_{1}\left(x^{\prime}\right)<f_{2}\left(x^{\prime}\right)<\cdots<f_{l}\left(x^{\prime}\right) \text { for all } x^{\prime} \in[-1,1]^{n-1} \text {. }
$$

These functions divide $\Omega^{+}$into $l+1$ regions

$$
\tilde{D}_{m}=\left\{x \in V \mid f_{m-1}\left(x^{\prime}\right)<x_{n}<f_{m}\left(x^{\prime}\right)\right\}, \quad 1 \leqq m \leqq l+1,
$$

where we have used the convention $f_{0} \equiv 0, f_{l+1} \equiv 1$.

Let $A^{(m)} \in C^{\mu}\left(\overline{\tilde{D}_{m}}\right), 1 \leqq m \leqq l+1$, be symmetric, positive definite matrixvalued functions, and let $A(\bar{x}) \in \overline{\mathcal{A}}(\bar{\lambda}, \bar{\Lambda})$ denote the matrix-valued function

$$
A(x)=A^{(m)}(x), \quad x \in \tilde{D}_{m}, \quad 1 \leqq m \leqq l+1 .
$$

Let $g^{(m)} \in C^{\mu}\left(\overline{\tilde{D}_{m}}, \mathbb{R}^{n}\right)$, and let $g$ denote the function

$$
g(x)=g^{(m)}(x), \quad x \in \tilde{D}_{m}, 1 \leqq m \leqq l+1 .
$$

Suppose $h$ is in $L^{\infty}\left(\Omega^{+}\right)$. We introduce a particular set of "strips"

$$
\Omega_{m}=\left\{x \in V \mid f_{m-1}\left(0^{\prime}\right)<x_{n}<f_{m}\left(0^{\prime}\right)\right\},
$$

and introduce the specific matrix-valued function $\bar{A} \in \overline{\mathcal{A}}(\bar{\lambda}, \bar{\Lambda})$

$$
\bar{A}(x)=A^{(m)}\left(0^{\prime}, f_{m-1}\left(0^{\prime}\right)\right), \quad x \in \Omega_{m}, \quad 1 \leqq m \leqq l+1,
$$

as well as the specific vector-valued function

$$
\bar{G}(x)=g^{(m)}\left(0^{\prime}, f_{m-1}\left(0^{\prime}\right)\right), \quad x \in \Omega_{m}, \quad 1 \leqq m \leqq l+1 .
$$

Proposition 6.5. Let $A \in \mathcal{A}(\bar{\lambda}, \bar{\Lambda}), \bar{A} \in \overline{\mathcal{A}}(\bar{\lambda}, \bar{\Lambda}), h, g$ and $\bar{G}$ be as defined just above. For any $q>n$, and any $0<\alpha^{\prime} \leqq \min \left\{\mu, \frac{\alpha}{(\alpha+1) q}\right\}$ there exist constants $C$ and $r_{0}$ such that if $u \in H^{1}\left(\Omega^{+}\right)$is a solution to

$$
\begin{aligned}
\partial_{i}\left(A_{i j} \partial_{j} u\right) & =h+\partial_{i} g_{i} & & \text { in } \Omega^{+}, \\
u & =\varphi & & \text { on }\left\{\left|x^{\prime}\right|<1, x_{n}=0\right\},
\end{aligned}
$$

with

$$
\|u\|_{L^{\infty}(\Omega)}+\|h\|_{L^{\infty}(\Omega)}+\max _{1 \leqq m \leqq l+1}\left\|g^{(m)}\right\|_{C^{\alpha^{\prime}}\left(\overline{\left.\tilde{D}_{m}\right)}\right.}+\|\varphi\|_{C^{1, \alpha^{\prime}\left(\left|x^{\prime}\right| \leqq 1\right)}} \leqq 1,
$$

then one may find a continuous, piecewise linear function, $p$, whose coefficients are bounded in absolute value by $C$, and which satisfies

$$
\partial_{i}\left(\bar{A}_{i j} \partial_{j} p(x)\right)=\partial_{i} \bar{G}_{i} \text { in } r_{0} \Omega^{+},
$$

and

$$
|u(x)-p(x)| \leqq C|x|^{1+\alpha^{\prime}}, \quad x \in r_{0} \Omega^{+} .
$$

The constants $C$ and $r_{0}$ depend on $n, \alpha^{\prime}, \alpha, q, \bar{\lambda}, \bar{\Lambda}$, the number $l, \max _{1 \leqq m \leqq l+1}$ $\left\|A^{(m)}\right\|_{C^{\alpha^{\prime}}\left(\overline{\tilde{D}_{m}}\right)}$, and $\max _{1 \leqq m \leqq l}\left\|f_{m}\right\|_{C^{1, \alpha}\left([-1,1]^{n-1}\right)}$. 
Proof. Based on the already stated local result the proof is a simple modification of that of Proposition 5.3.

Proof of Theorem 1.2. We can locally make a $C^{1, \alpha}$ diffeomorphism to flatten the boundary without changing the form of the equation. We may suppose that the domain $D$ locally coincides with the halfspace $x_{n}>0$. We can now apply the exact same technique as that used to prove Theorem 1.1, only this time we use Proposition 6.5 in place of Proposition 5.3.

\section{Hölder estimates}

In this section we examine the regularity properties of solutions, when the boundary data are only known to be Hölder continuous, and $h$ and $g$ are only known to be bounded. That is to say, instead of (3) and(5) we suppose

$$
h \in L^{\infty}(D), \quad g=\left(g_{1}, \cdots, g_{n}\right) \in L^{\infty}(D),
$$

and, for some $0<v<1$,

$$
\varphi \in C^{v}(\partial D)
$$

The following theorems are analogous to Theorem 1.1 and Theorem 1.2.

Theorem 7.1. Let A satisfy (1), and let h and $g$ satisfy (76). There exists a constant $C$ depending only on $D, n, \alpha, \mu, v, \varepsilon, \bar{\lambda}, \bar{\Lambda},\left\|A^{(m)}\right\|_{C^{\mu}\left(\bar{D}_{m}\right)}$ and the $C^{1, \alpha}$ modulus of $\cup_{m=1}^{L} \partial D_{m}$, such that if $u \in H^{1}(D)$ is a solution to

$$
\partial_{i}\left(A_{i j} \partial_{j} u\right)=h+\partial_{i} g_{i} \text { in } D,
$$

then

$$
\|u\|_{C^{\nu}\left(D_{\varepsilon}\right)} \leqq C\left(\|u\|_{L^{\infty}(D)}+\|h\|_{L^{\infty}(D)}+\|g\|_{L^{\infty}(D)}\right),
$$

where $D_{\varepsilon}=\{x \in D \mid \operatorname{dist}(x, \partial D)>\varepsilon\}$.

Theorem 7.2. Let A satisfy (1), let $h, g$ and $\varphi$ satisfy (76) and (77), and suppose $r>$ 0 . There exists a constant $C$ depending only on $D, n, \alpha, \mu, v, r, \bar{\lambda}, \bar{\Lambda},\left\|A^{(m)}\right\|_{C^{\mu}\left(\bar{D}_{m}\right)}$ and the $C^{1, \alpha}$ modulus of $\cup_{m=1}^{L} \partial D_{m}$, such that if, for some $\bar{x} \in \partial D, u \in H^{1}(D \cap$ $\left.B_{2 r}(\bar{x})\right)$ is a solution to

$$
\begin{aligned}
\partial_{i}\left(A_{i j} \partial_{j} u\right) & =h+\partial_{i} g_{i} & & \text { in } D \cap B_{2 r}(\bar{x}), \\
u & =\varphi & & \text { on } \partial D \cap B_{2 r}(\bar{x}),
\end{aligned}
$$

then

$$
\begin{aligned}
\|u\|_{C^{\nu}\left(\bar{D} \cap B_{r}(\bar{x})\right)} \leqq C\left(\|u\|_{L^{\infty}\left(D \cap B_{2 r}(\bar{x})\right)}+\|\varphi\|_{C^{\nu}\left(\partial D \cap B_{2 r}(\bar{x})\right)}\right. \\
\left.+\|h\|_{L^{\infty}\left(D \cap B_{2 r}(\bar{x})\right)}+\|g\|_{L^{\infty}\left(D \cap B_{2 r}(\bar{x})\right)}\right) .
\end{aligned}
$$


The following global $C^{v}$ estimate is a consequence of Theorem 7.1, Theorem 7.2 , and the maximum priciple.

Corollary 7.3. Let A satisfy (1), and let $h, g$ and $\varphi$ satisfy (76) and (77). There exists a constant $C$ depending only on $D, n, \alpha, \mu, v, \bar{\lambda}, \bar{\Lambda},\left\|A^{(m)}\right\|_{C^{\mu}\left(\bar{D}_{m}\right)}$ and the $C^{1, \alpha}$ modulus of $\cup_{m=1}^{L} \partial D_{m}$, such that if $u \in H^{1}(D)$ is a solution to

$$
\begin{aligned}
\partial_{i}\left(A_{i j} \partial_{j} u\right) & =h+\partial_{i} g_{i} & & \text { in } D, \\
u & =\varphi & & \text { on } \partial D,
\end{aligned}
$$

then

$$
\|u\|_{C^{\nu}(\bar{D})} \leqq C\left(\|\varphi\|_{C^{\nu}(\partial D)}+\|h\|_{L^{\infty}(D)}+\|g\|_{L^{\infty}(D)}\right)
$$

Remark 7.1. In view of the De Giorgi-Nash estimates, the term $\|u\|_{L^{\infty}\left(D \cap B_{2 r}(\bar{x})\right)}$ in the estimate of Theorem 7.2 can be replaced by $\|u\|_{L^{2}\left(D \cap B_{2 r}(\bar{x})\right)}$.

In the following we use the same notation as in the last section. We assume

$$
\begin{gathered}
g, h \in L^{\infty}\left(\Omega^{+}\right), \text {and } \\
\varphi \in C^{v}\left(\left|x^{\prime}\right|<1\right) .
\end{gathered}
$$

The proofs of Theorem 7.1 and Theorem 7.2 are quite similar to the proofs we have already provided. Since the proof of Theorem 7.1 is comparatively the easiest, we shall here only concern ourselves with the proof of Theorem 7.2. In order to prove this theorem we need to establish

Proposition 7.4. Let A satisfy (74), and let $g$, h and $\varphi$ satisfy (78) and (79). Suppose $q>n$ and $\varepsilon>0$. There exists a constant $C$, such that if $u \in H^{1}\left(\Omega^{+}\right)$is a solution to

$$
\begin{aligned}
\partial_{i}\left(A_{i j} \partial_{j} u\right) & =h+\partial_{i} g_{i} & & \text { in } \Omega^{+}, \\
u & =\varphi & & \text { on }\left\{\left|x^{\prime}\right|<1, x_{n}=0\right\},
\end{aligned}
$$

then

$$
\|u\|_{C^{\nu}\left((1-\varepsilon) \overline{\left.\Omega^{+}\right)}\right.} \leqq C\left(\|u\|_{L^{\infty}\left(\Omega^{+}\right)}+\|\varphi\|_{C^{v}\left(\left|x^{\prime}\right|<1\right)}+\|h\|_{Y^{v-1, q / 2}}+\|g\|_{Y^{v, q}}\right) .
$$

The constant $C$ depends only on $D, n, \varepsilon, q, \mu, v, \bar{\lambda}, \bar{\Lambda}$, the number $l$, $\max _{1 \leqq m \leqq l+1}$ $\left\|A^{(m)}\right\|_{C^{\mu}\left(\bar{\Omega}_{m}\right)}$, and $\max _{1 \leqq m \leqq l}\left\|f_{m}\right\|_{C^{1, \alpha}\left((-1,1)^{n-1}\right)}$.

The proof of Proposition 7.4 follows the same lines as the proof of Theorem 1.1. It is in fact somewhat simpler, and we shall therefore only state some relevant lemmas and sketch the proof. First we need a lemma whose proof is similar to that of Lemma 6.3. 
Lemma 7.5. Suppose $A \in \mathcal{A}(\bar{\lambda}, \bar{\Lambda}), \bar{A} \in \overline{\mathcal{A}}(\bar{\lambda}, \bar{\Lambda}), g=\left(g_{1}, \cdots, g_{n}\right) \in L^{q}\left(\Omega^{+}\right)$, and $h \in L^{q / 2}\left(\Omega^{+}\right)$for some $q>n$. Let $u \in H^{1}\left(\Omega^{+}\right)$be a solution to

$$
\partial_{i}\left(A_{i j} \partial_{j} u\right)=h+\partial_{i} g_{i} \text { in } \Omega^{+},
$$

with

$$
u=\varphi \quad \text { on }\left\{\left|x^{\prime}\right|<1, x_{n}=0\right\}
$$

and

$$
\|u\|_{L^{\infty}\left(\Omega^{+}\right)} \leqq 1 .
$$

There exist positive constants $\gamma \leqq 1$ and $C$ (depending on $q, n, \bar{\lambda}$ and $\bar{\Lambda}$ ) such that whenever

$$
\left(f_{\Omega^{+}}|A-\bar{A}|^{q} d x\right)^{1 / q} \leqq \varepsilon
$$

for some $\varepsilon>0$, then we may find a function $v \in H^{1}$ with

$$
\begin{aligned}
\partial_{i}\left(\bar{A}_{i j}(x) \partial_{j} v\right) & =0 & & \text { in } \frac{3}{4} \Omega^{+}, \\
v & =\varphi\left(0^{\prime}\right) & & \text { on }\left\{\left|x^{\prime}\right|<\frac{3}{4}, x_{n}=0\right\},
\end{aligned}
$$

and with

$$
\begin{aligned}
\|u-v\|_{L^{\infty}\left(\frac{1}{2} \Omega^{+}\right)} \leqq & C\left(\left[1+\left|\varphi\left(0^{\prime}\right)\right|\right] \varepsilon^{\gamma}\right. \\
& \left.+\|g\|_{L^{q}\left(\Omega^{+}\right)}+\|h\|_{L^{q / 2}\left(\Omega^{+}\right)}+\left\|\varphi-\varphi\left(0^{\prime}\right)\right\|_{L^{\infty}\left(\left|x^{\prime}\right|<1\right)}\right) .
\end{aligned}
$$

Next we need a proposition similar to Proposition 6.4.

Proposition 7.6. Suppose $A \in \mathcal{A}(\bar{\lambda}, \bar{\Lambda})$ and $\bar{A} \in \overline{\mathcal{A}}(\bar{\lambda}, \bar{\Lambda})$, relative to the hyperplanes $x_{n}=c_{m}, 0 \leqq m \leqq l+1$. Suppose $g=\left(g_{1}, \cdots, g_{n}\right) \in L^{q}\left(\Omega^{+}\right)$, and $h \in L^{q / 2}\left(\Omega^{+}\right)$for some $q>n$. Let $u \in H^{1}\left(\Omega^{+}\right)$denote a solution to

$$
\begin{aligned}
\partial_{i}\left(A_{i j}(x) \partial_{j} u\right) & =h+\partial_{i} g_{i} & & \text { in } \Omega^{+}, \\
u & =\varphi & & \text { on }\left\{\left|x^{\prime}\right|<1, x_{n}=0\right\},
\end{aligned}
$$

with

$$
\|u\|_{L^{\infty}\left(\Omega^{+}\right)} \leqq 1 .
$$

There exist constants in the range $0<\sigma<\frac{1}{4}, \varepsilon_{0}>0$, and $C>0$ (depending on $n, q, \mu, \nu$, and the ellipticity constants $\bar{\lambda}, \bar{\Lambda})$ such that if

$$
\begin{gathered}
\|A-\bar{A}\|_{Y^{1, q}} \leqq \varepsilon_{0}, \\
\|g\|_{Y^{v, q}}+\|h\|_{Y^{v-1, q / 2}}+\left\|\varphi-\varphi\left(0^{\prime}\right)\right\|_{C^{v}\left(\left|x^{\prime}\right|<1\right)} \leqq \varepsilon_{0},
\end{gathered}
$$


and

$$
\|\varphi\|_{C^{v}\left(\left|x^{\prime}\right|<1\right)} \leqq 1
$$

then we have

$$
\|u-u(0)\|_{L^{\infty}\left(\sigma^{k} \Omega^{+}\right)} \leqq\left(\sigma^{k}\right)^{v}
$$

Consequently,

$$
|u(x)-u(0)| \leqq C|x|^{\nu}, \quad x \in \Omega
$$

Proof. The proof of this proposition is similar to that of Proposition 6.4. We also proceed by induction. The choices of $\sigma$ and $\varepsilon_{0}$ will be made in the process. We first prove that the estimate $\left(P_{k}\right)$ holds for $k=1$. Using Lemma 7.5, we establish the existence of a function $v$ that solves

$$
\partial_{i}\left(\bar{A}_{i j}(x) \partial_{j} v\right)=0 \quad \text { in } \frac{3}{4} \Omega^{+},
$$

with

$$
v=u(0) \quad \text { on }\left\{x_{n}=0\right\},
$$

and for which

$$
\|u-v\|_{L^{\infty}\left(\frac{1}{2} \Omega^{+}\right)} \leqq C\left(\varepsilon_{0}^{\gamma}+2 \varepsilon_{0}\right) \leqq C \varepsilon_{0}^{\gamma} .
$$

According to this last estimate we also have

$$
\|v\|_{L^{\infty}\left(\frac{1}{2} \Omega^{+}\right)} \leqq C
$$

and thus in view of Proposition 6.1

$$
|v(x)-u(0)| \leqq C|x|, \quad x \in \Omega^{+} .
$$

Select $\sigma$ so that

$$
C \sigma \leqq \frac{1}{2} \sigma^{v}
$$

and then select $\varepsilon_{0}$ so that

$$
C \varepsilon_{0}^{\gamma} \leqq \frac{1}{2} \sigma^{\nu}
$$

to obtain $\left(P_{1}\right)$.

Now we suppose $\left(P_{1}\right), \ldots,\left(P_{k}\right)$ hold and proceed to verify $\left(P_{k+1}\right)$. Consider

$$
W(x)=\frac{u\left(\sigma^{k} x\right)-u(0)}{\left(\sigma^{k}\right)^{v}}, \quad x \in \Omega^{+} .
$$

This function satisfies

$$
\|W\|_{L^{\infty}\left(\Omega^{+}\right)} \leqq 1
$$

and

$$
\partial_{i}\left(A_{i j}\left(\sigma^{k} x\right) \partial_{j} W\right)=h^{(k)}(x)+\partial_{i} g_{i}^{(k)}(x)
$$


with

$$
h^{(k)}(x)=\frac{h\left(\sigma^{k} x\right)}{\left(\sigma^{k}\right)^{v-2}},
$$

and

$$
g_{i}^{(k)}(x)=\frac{g_{i}\left(\sigma^{k} x\right)}{\left(\sigma^{k}\right)^{v-1}} .
$$

A simple calculation, using the hypothesis, gives

$$
\left\|h^{(k)}\right\|_{L^{q / 2}\left(\Omega^{+}\right)}+\left\|g_{i}^{(k)}\right\|_{L^{q}\left(\Omega^{+}\right)} \leqq C \varepsilon_{0},
$$

and

$$
\left(\int_{\Omega^{+}}\left|A_{i j}\left(\sigma^{k} x\right)-\bar{A}_{i j}\left(\sigma^{k} x\right)\right|^{q}\right)^{1 / q} \leqq C \varepsilon_{0} .
$$

Since $W(0)=0$ and $\left|W\left(x^{\prime}, 0\right)-W(0)\right|=\left|\left(\sigma^{-k}\right)^{v}\right| \varphi\left(\sigma^{k} x^{\prime}\right)-\varphi\left(0^{\prime}\right) \mid \leqq \varepsilon_{0}$, we now infer from Lemma 7.5 the existence of a solution to

$$
\partial_{i}\left(\bar{A}_{i j}\left(\sigma^{k} x\right) \partial_{j} Z\right)=0 \quad \text { in } \frac{3}{4} \Omega^{+},
$$

and

$$
Z\left(x^{\prime}, 0\right)=0
$$

and with the property that

$$
\|W-Z\|_{L^{\infty}\left(\frac{1}{2} \Omega^{+}\right)} \leqq C \varepsilon_{0}^{\gamma} .
$$

According to the last estimate we also have

$$
\|Z\|_{L^{\infty}\left(\frac{1}{2} \Omega^{+}\right)} \leqq C,
$$

and thus, in view of Proposition 6.1,

$$
|Z(x)| \leqq C|x| \quad x \in \frac{1}{4} \Omega^{+} .
$$

It follows that

$$
|W(x)| \leqq C \varepsilon_{0}^{\gamma}+C|x|, \quad x \in \frac{1}{4} \Omega^{+} .
$$

Now, select even smaller $\sigma$ and $\varepsilon_{0}$ in the same fashion as before, so as to obtain

$$
|W(x)| \leqq \sigma^{\nu}, \quad x \in \sigma \Omega^{+} .
$$

This implies

$$
\|u-u(0)\|_{L^{\infty}\left(\sigma^{k+1} \Omega^{+}\right)} \leqq\left(\sigma^{k+1}\right)^{v},
$$

which is exactly $(P)_{k+1}$.

The derivation of Proposition 7.4 from Proposition 7.6 and the derivation of Theorem 7.2 from Proposition 7.4 are very similar to the derivations in earlier sections. We leave the details to the reader. 


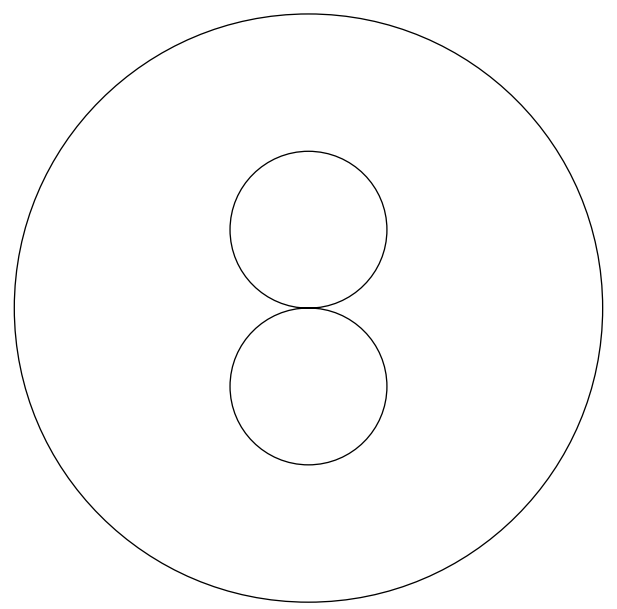

Fig. 2. Two touching disks.

\section{An example of two touching disks}

Consider the two-dimensional geometric situation illustrated in Fig. 2: the domain $\Omega$ is now given by $\Omega=\left\{x:|x|<R_{0}\right\}\left(R_{0}>2\right)$, and it contains exactly two circular inclusions of radius 1 , centered at $(0, \pm 1)$. In this section we use $|\cdot|$ to denote the Euclidean norm of an element in $\mathbb{R}^{2}$, as well as the modulus of a complex number. We consider the boundary value problem $\partial_{i}\left(a(x) \partial_{i} u\right)=0$ in $\Omega$, $u=g$ on the boundary. The coefficient $a$ is given by:

$$
\begin{array}{ll}
a(x)=1 & \text { for } x \text { outside the two inclusions, } \\
a(x)=a_{0} & \text { for } x \text { inside the two inclusions }
\end{array}
$$

with $0<a_{0}<\infty$.

The solution $u$ is clearly infinitely often differentiable inside the open set $\Omega \backslash$ $(\{x:|x-(0,1)|=1\} \cup\{x:|x-(0,-1)|=1\})$; we shall show that any derivative of $u$ is furthermore uniformly bounded inside this set (provided we stay away from the boundary of $\Omega$ ). To be precise

Proposition 8.1. Suppose $R_{0}$ is sufficiently large. Let $g$ be in $H^{1 / 2}(\partial \Omega)$, and let $u \in H^{1}(\Omega)$ denote the solution to

$$
\partial_{i}\left(a(x) \partial_{i} u\right)=0 \quad \text { in } \Omega, \quad u=g \quad \text { on } \partial \Omega .
$$

Then

$$
\begin{aligned}
& u \in C^{\infty}(K \cap\{x:|x-(0,1)| \geqq 1\} \cap\{x:|x-(0,-1)| \geqq 1\}), \\
& u \in C^{\infty}(\{x:|x-(0,1)| \leqq 1\}), \quad \text { and } u \in C^{\infty}(\{x:|x-(0,-1)| \leqq 1\}),
\end{aligned}
$$

for any compact set $K \subset \Omega$. 
Remark 8.1. The above regularity statement about $u$ also asserts that the limiting value of any derivative $D^{\mathbf{m}} u(x)$, as $x$ approaches $(0,0)$ from within the set $\{x: \mid x-$ $(0,1) \mid>1\} \cap\{x:|x-(0,-1)|>1\}$, exists (is finite) and is the same whether $x$ approaches the origin through the left cusp or through the right cusp. For $m_{2}>0$ this limiting value is of course in general different from the limiting value we obtain as $x$ approaches the origin through the upper disk (or through the lower disk). By an extension of our proof of Proposition 8.1 it is possible to see that the restriction of $u$ to any of the three closed sets may indeed be extended as a $C^{\infty}$ function to an open neighborhood (or for that matter to all of $\Omega$ ) - but these three extensions do not coincide.

Remark 8.2. Let $u_{\varepsilon}$ denote the corresponding solution when the the two circular inclusions are centered at $(0, \pm(1+\varepsilon))$. The fact that $u\left(=u_{0}\right)$ is as smooth as one could possibly hope for, does not automatically imply that all derivatives of $u_{\varepsilon}$ are uniformly bounded, independent of $\varepsilon$, in each of the three sets $\{x: \mid x-(0, \pm(1+$ $\varepsilon)) \mid \leqq 1\}$ and $K \cap\{x:|x-(0,1+\varepsilon)| \geqq 1\} \cap\{x:|x-(0,-1-\varepsilon)| \geqq 1\}$. When " $a_{0}=\infty$ " (i.e., when the boundary condition $u_{\varepsilon}=$ const is imposed on the boundary of each of the two inclusions, such reasoning is indeed wrong. We do feel, however, that for $0<a_{0}<\infty$ (as is the case here) the smoothness exhibited by $u_{0}$ makes it quite likely that the $u_{\varepsilon}$ have piecewise defined, uniformly bounded derivatives of any order (away from $\partial \Omega$ ). If anything, the smoothness exhibited by $u_{0}$ certainly makes it much more difficult to construct examples contradicting this type of behavior.

It suffices to prove Proposition 8.1 for solutions that are even with respect to the $x_{2}$ axis. A standard duality argument transforms this into a proof of the same fact for solutions that are odd with respect to the $x_{2}$ axis. By decomposing any solution into a sum of its even and odd parts the proposition now follows in general.

In order to verify Proposition 8.1 for solutions that are even with respect to the $x_{2}$ axis, it is essential to construct a whole family of solutions to

$$
\partial_{i}\left(a(x) \partial_{i} u\right)=0 \quad \text { in } \Omega .
$$

Let $-1<\alpha<1$ denote the ratio $\alpha=\frac{a_{0}-1}{a_{0}+1}$. Let $\phi(\cdot)$ be analytic in $\mathcal{C} \backslash(0,0)$, and suppose $\phi$ has the two additional properties

$$
\begin{aligned}
\phi(\bar{\zeta}) & =\overline{\phi(\zeta)} \\
|\phi(\zeta)| & \leqq C \beta^{|\operatorname{Re} \zeta|}, \quad \frac{1}{2}<|\operatorname{Re} \zeta|,
\end{aligned}
$$

for some $0<\beta<|\alpha|^{-1}$. Let $\phi_{o}(\zeta)=\frac{1}{2}(\phi(\zeta)-\phi(-\zeta))$ and $\phi_{e}(\zeta)=\frac{1}{2}(\phi(\zeta)+$ $\phi(-\zeta))$ denote the odd and the even part of $\phi$, respectively. We now define

$$
\begin{aligned}
& \Phi_{o}(\zeta)=-\frac{2}{a_{0}+1} \sum_{k=0}^{\infty} \alpha^{k} \phi_{o}(k-\zeta), \quad \operatorname{Re} \zeta<-\frac{1}{2}, \\
& \Phi_{o}(\zeta)=\phi_{o}(\zeta)+\sum_{k=1}^{\infty} \alpha^{k}\left[\phi_{o}(k+\zeta)-\phi_{o}(k-\zeta)\right], \quad-\frac{1}{2}<\operatorname{Re} \zeta<\frac{1}{2}, \\
& \Phi_{o}(\zeta)=\frac{2}{a_{0}+1} \sum_{k=0}^{\infty} \alpha^{k} \phi_{o}(k+\zeta), \quad \frac{1}{2}<\operatorname{Re} \zeta,
\end{aligned}
$$


as well as

$$
\begin{aligned}
& \Phi_{e}(\zeta)=\frac{2}{a_{0}+1} \sum_{k=0}^{\infty}(-\alpha)^{k} \phi_{e}(k-\zeta), \quad \operatorname{Re} \zeta<-\frac{1}{2} \\
& \Phi_{e}(\zeta)=\phi_{e}(\zeta)+\sum_{k=1}^{\infty}(-\alpha)^{k}\left[\phi_{e}(k+\zeta)+\phi_{e}(k-\zeta)\right], \quad-\frac{1}{2}<\operatorname{Re} \zeta<\frac{1}{2} \\
& \Phi_{e}(\zeta)=\frac{2}{a_{0}+1} \sum_{k=0}^{\infty}(-\alpha)^{k} \phi_{e}(k+\zeta), \quad \frac{1}{2}<\operatorname{Re} \zeta
\end{aligned}
$$

and

$$
\Phi(\zeta)=\frac{1}{2}\left(\Phi_{o}(\zeta)+\Phi_{e}(\zeta)\right)
$$

We use the notation $\mathbf{i}$ for the imaginary unit.

Proposition 8.2. The functions $u_{o}\left(x_{1}, x_{2}\right)=\operatorname{Re} \Phi_{o}(\mathbf{i} / z), u_{e}\left(x_{1}, x_{2}\right)=\operatorname{Re} \Phi_{e}(\mathbf{i} / z)$, and $u\left(x_{1}, x_{2}\right)=\operatorname{Re} \Phi(\mathbf{i} / z)$ (with $z=x_{1}+\mathbf{i} x_{2}$ ) satisfy

$$
\partial_{i}\left(a(x) \partial_{i} u_{o}\right)=\partial_{i}\left(a(x) \partial_{i} u_{e}\right)=\partial_{i}\left(a(x) \partial_{i} u\right)=0 .
$$

All of these functions are even with respect to the $x_{2}$ axis. The function $u_{o}$ is odd with respect to the $x_{1}$ axis, the function $u_{e}$ is even with respect to the $x_{1}$ axis. The fuction $u$ is related to $u_{o}$ and $u_{e}$ by $u\left(x_{1}, x_{2}\right)=\frac{1}{2}\left(u_{o}\left(x_{1}, x_{2}\right)+u_{e}\left(x_{1}, x_{2}\right)\right)$.

Proof. The symmetry properties of $u_{o}$ and $u_{e}$ are obvious, and so is the alternate representation for $u$. We shall verify that $u_{o}$ solves the equation $\partial_{i}\left(a(x) \partial_{i} u_{o}\right)=0$, the verification for $u_{e}$ is similar (and it then follows immediately that $u$ is also a solution). The conformal mapping $z \rightarrow \zeta=\mathbf{i} / z$ maps the (extended) complex plane with the two unit circles centered at $\pm \mathbf{i}$ onto the (extended) complex plane with the two vertical lines $\operatorname{Re} \zeta=-\frac{1}{2}$ and $\operatorname{Re} \zeta=\frac{1}{2}$. The upper circle is mapped to $\operatorname{Re} \zeta=\frac{1}{2}$, its interior is mapped to $\frac{1}{2}<\operatorname{Re} \zeta$, the lower circle is mapped to $\operatorname{Re} \zeta=-\frac{1}{2}$, and its interior is mapped to $\operatorname{Re} \zeta<-\frac{1}{2}$. To verify that $u_{o}$ solves the equation $\partial_{i}\left(a(x) \partial_{i} u_{o}\right)=0$ it thus suffices to verify that $\operatorname{Re} \Phi_{o}(\zeta)$ solves the equation

$$
\partial_{\eta_{1}}\left(A\left(\eta_{1}\right) \partial_{\eta_{1}} \operatorname{Re} \Phi_{o}\right)+\partial_{\eta_{2}}\left(A\left(\eta_{1}\right) \partial_{\eta_{2}} \operatorname{Re} \Phi_{o}\right)=0
$$

with $A$ given by $A\left(\eta_{1}\right)=a_{0}$ for $\frac{1}{2}<\left|\eta_{1}\right|$, and $A\left(\eta_{1}\right)=1$ for $\left|\eta_{1}\right|<\frac{1}{2}$, and $\zeta=\eta_{1}+\mathbf{i} \eta_{2}$.

Since $\operatorname{Re} \Phi_{o}$ is harmonic in each of the three strips $\eta_{1}<-\frac{1}{2},-\frac{1}{2}<\eta_{1}<\frac{1}{2}$ and $\frac{1}{2}<\eta_{1}$, it suffices to verify that $\operatorname{Re} \Phi_{o}$, and the conormal derivative $A\left(\eta_{1}\right) \partial_{\eta_{1}} \operatorname{Re} \Phi_{o}$ are continuous across the lines $\eta_{1}=-\frac{1}{2}$ and $\eta_{1}=\frac{1}{2}$. Because of the relationship between the real and the imaginary parts of an analytic function (they are harmonic conjugates) these continuity conditions may be verified by checking that

$\operatorname{Re} \Phi_{o}$ and $A\left(\eta_{1}\right) \partial_{\eta_{2}} \operatorname{Im} \Phi_{o} \quad$ are continuous across the lines $\eta_{1}= \pm \frac{1}{2}$ 
We may verify the second of these properties by checking that

$$
A\left(\eta_{1}\right) \operatorname{Im} \Phi_{o} \text { is continuous across the lines } \eta_{1}= \pm \frac{1}{2} \text {. }
$$

We present the calculations associated with the continuity properties across the line $\eta_{1}=\frac{1}{2}$, the continuity properties across the line $\eta_{1}=-\frac{1}{2}$ follow by symmetry.

In order to verify that $\operatorname{Re} \Phi_{o}$ is continuous (across the line $\eta_{1}=\frac{1}{2}$ ) it follows from the definition (82) that we must verify

$$
\begin{aligned}
\phi_{o}(\zeta)+\overline{\phi_{o}(\zeta)} & +\sum_{k=1}^{\infty} \alpha^{k}\left[\phi_{o}(k+\zeta)+\overline{\phi_{o}(k+\zeta)}-\left(\phi_{o}(k-\zeta)+\overline{\phi_{o}(k-\zeta)}\right)\right] \\
& =\frac{2}{a_{0}+1} \sum_{k=0}^{\infty} \alpha^{k}\left[\phi_{o}(k+\zeta)+\overline{\phi_{o}(k+\zeta)}\right] \quad \text { at } \operatorname{Re} \zeta=\frac{1}{2}, \quad
\end{aligned}
$$

or equivalently

$$
\begin{aligned}
\phi_{o}(\zeta)+\phi_{o}(\bar{\zeta})+ & \sum_{k=1}^{\infty} \alpha^{k}\left[\phi_{o}(k+\zeta)+\phi_{o}(k+\bar{\zeta})\right] \\
& \left.-\alpha \sum_{k=1}^{\infty} \alpha^{k-1}\left(\phi_{o}(k-\zeta)+\phi_{o}(k-\bar{\zeta})\right)\right] \\
= & \frac{2}{a_{0}+1} \sum_{k=0}^{\infty} \alpha^{k}\left[\phi_{o}(k+\zeta)+\phi_{o}(k+\bar{\zeta})\right] \quad \text { at } \operatorname{Re} \zeta=\frac{1}{2}
\end{aligned}
$$

For $\operatorname{Re} \zeta=\frac{1}{2}$ we have $k-\zeta=k-1+\bar{\zeta}$ and $k-\bar{\zeta}=k-1+\zeta$, and therefore the left-hand side in the identity (85) equals

$$
(1-\alpha) \sum_{k=0}^{\infty} \alpha^{k}\left[\phi_{o}(k+\zeta)+\phi_{o}(k+\bar{\zeta})\right]
$$

this immediately verifies the identity (84), since $1-\alpha=\frac{2}{a_{0}+1}$.

To verify that $A\left(\eta_{1}\right) \operatorname{Im} \Phi_{o}$ is continuous across the line $\eta_{1}=\frac{1}{2}$ we must ascertain that

$$
\begin{aligned}
\phi_{o}(\zeta)-\overline{\phi_{o}(\zeta)} & +\sum_{k=1}^{\infty} \alpha^{k}\left[\phi_{o}(k+\zeta)-\overline{\phi_{o}(k+\zeta)}-\left(\phi_{o}(k-\zeta)-\overline{\phi_{o}(k-\zeta)}\right)\right] \\
& =\frac{2 a_{0}}{a_{0}+1} \sum_{k=0}^{\infty} \alpha^{k}\left[\phi_{o}(k+\zeta)-\overline{\phi_{o}(k+\zeta)}\right] \quad \text { at } \operatorname{Re} \zeta=\frac{1}{2}, \quad
\end{aligned}
$$

(remember that $A\left(\eta_{1}\right)=a_{0}$ for $\frac{1}{2}<\eta_{1}=\operatorname{Re} \zeta$, and $A\left(\eta_{1}\right)=1$ for $-\frac{1}{2}<\eta_{1}=$ $\operatorname{Re} \zeta<\frac{1}{2}$ ). The above identity is equivalent to 


$$
\begin{aligned}
\phi_{o}(\zeta)-\phi_{o}(\bar{\zeta})+ & \sum_{k=1}^{\infty} \alpha^{k}\left[\phi_{o}(k+\zeta)-\phi_{o}(k+\bar{\zeta})\right]-\alpha \sum_{k=1}^{\infty} \alpha^{k-1}\left[\phi_{o}(k-\zeta)-\phi_{o}(k-\bar{\zeta})\right] \\
& =\frac{2 a_{0}}{a_{0}+1} \sum_{k=0}^{\infty} \alpha^{k}\left[\phi_{o}(k+\zeta)-\phi_{o}(k+\bar{\zeta})\right] \quad \text { at } \operatorname{Re} \zeta=\frac{1}{2} .
\end{aligned}
$$

Since $k-\zeta=k-1+\bar{\zeta}$ and $k-\bar{\zeta}=k-1+\zeta$ for $\operatorname{Re} \zeta=\frac{1}{2}$, we conclude that the left-hand side in the identity (87) equals

$$
(1+\alpha) \sum_{k=0}^{\infty} \alpha^{k}\left[\phi_{o}(k+\zeta)-\phi_{o}(k+\bar{\zeta})\right]
$$

this immediately verifies the identity (86), since $1+\alpha=\frac{2 a_{0}}{a_{0}+1}$.

We now apply Proposition 8.2 and the definitions preceeding it to the functions $\phi^{(j)}(\zeta)=R_{0}^{-j}(1 / \zeta)^{j}, j=0,1,2, \ldots$, where $R_{0}$ is the radius of the disk $\Omega$. We may apply Proposition 8.2 since these $\phi^{(j)}$ satisfy the requirements (80) and (81), the latter with $\beta=1<|\alpha|^{-1}$. We thus create a family $\left\{u_{j}\right\}_{j=0}^{\infty}$ of solutions to our elliptic boundary value problem of the form $u_{j}\left(x_{1}, x_{2}\right)=R_{0}^{-j} \operatorname{Re} \Psi_{j}(z)$ with

$$
\begin{aligned}
\Psi_{j}(z)= & -\frac{2}{a_{0}+1} \sum_{k=0}^{\infty} \alpha^{k} \frac{z^{j}}{(k z-\mathbf{i})^{j}} \quad \text { in }\{z:|z+\mathbf{i}|<1\}, \\
\Psi_{j}(z)= & (-1)^{\frac{j+1}{2}} \mathbf{i} z^{j}+\sum_{k=1}^{\infty} \alpha^{k}\left[\frac{z^{j}}{(k z+\mathbf{i})^{j}}-\frac{z^{j}}{(k z-\mathbf{i})^{j}}\right] \\
& \text { in }\{z:|z+\mathbf{i}|>1 \text { and }|z-\mathbf{i}|>1\}, \\
\Psi_{j}(z)= & \frac{2}{a_{0}+1} \sum_{k=0}^{\infty} \alpha^{k} \frac{z^{j}}{(k z+\mathbf{i})^{j}} \quad \text { in }\{z:|z-\mathbf{i}|<1\},
\end{aligned}
$$

for $j$ odd, and

$$
\begin{aligned}
\Psi_{j}(z)= & \frac{2}{a_{0}+1} \sum_{k=0}^{\infty}(-\alpha)^{k} \frac{z^{j}}{(k z-\mathbf{i})^{j}} \text { in }\{z:|z+\mathbf{i}|<1\}, \\
\Psi_{j}(z)= & (-1)^{j / 2} z^{j}+\sum_{k=1}^{\infty}(-\alpha)^{k}\left[\frac{z^{j}}{(k z+\mathbf{i})^{j}}+\frac{z^{j}}{(k z-\mathbf{i})^{j}}\right] \\
& \text { in }\{z:|z+\mathbf{i}|>1 \text { and }|z-\mathbf{i}|>1\}, \\
\Psi_{j}(z)= & \frac{2}{a_{0}+1} \sum_{k=0}^{\infty}(-\alpha)^{k} \frac{z^{j}}{(k z+\mathbf{i})^{j}} \text { in }\{z:|z-\mathbf{i}|<1\},
\end{aligned}
$$

for $j$ even. It requires some calculation, but it is absolutely straightforward, to obtain the following estimates concerning the functions $\Psi_{j}(z)$, for $|z| \leqq 1$. 
Proposition 8.3. Given any integer $0 \leqq m$ there exists a constant $C_{m}$, independent of $j$, so that the functions $\Psi_{j}$ satisfy

$$
\left|\left(\frac{d}{d z}\right)^{m} \Psi_{j}(z)\right| \leqq C_{m}(j+m)^{m}
$$

in each of the three regions

$$
\begin{gathered}
\{z:|z| \leqq 1,|z+\mathbf{i}| \geqq 1, \quad|z-\mathbf{i}| \geqq 1\}, \\
\{z:|z| \leqq 1,|z+\mathbf{i}| \leqq 1\}, \quad \text { and }\{z:|z| \leqq 1,|z-\mathbf{i}| \leqq 1\} .
\end{gathered}
$$

As a direct consequence of these estimates we get the following result.

Proposition 8.4. Given any multi-index $\mathbf{m}$ there exist a constant $C_{\mathbf{m}}$, independent of $j$ and $R_{0}$, so that the functions $u_{j}$ satisfy

$$
\left|D^{\mathbf{m}} u_{j}\left(x_{1}, x_{2}\right)\right| \leqq C_{\mathbf{m}} R_{0}^{-j}(j+|\mathbf{m}|)^{|\mathbf{m}|},
$$

in each of the three regions

$$
\begin{gathered}
\left\{\left(x_{1}, x_{2}\right):\left|\left(x_{1}, x_{2}\right)\right| \leqq 1,\left|\left(x_{1}, x_{2}\right)-(0,-1)\right| \geqq 1,\left|\left(x_{1}, x_{2}\right)-(0,1)\right| \geqq 1\right\}, \\
\left\{\left(x_{1}, x_{2}\right):\left|\left(x_{1}, x_{2}\right)\right| \leqq 1,\left|\left(x_{1}, x_{2}\right)-(0,-1)\right| \leqq 1\right\}, \quad \text { and } \\
\left\{\left(x_{1}, x_{2}\right):\left|\left(x_{1}, x_{2}\right)\right| \leqq 1,\left|\left(x_{1}, x_{2}\right)-(0,1)\right| \leqq 1\right\} .
\end{gathered}
$$

Let $L_{\text {sym }}^{2}\left(\left\{x:|x|=R_{0}\right\}\right)$ denote the set of real valued $L^{2}$ functions that are even with respect to the $x_{2}$ axis. We use a similar notation for the Sobolev spaces $H^{s}, 0 \leqq s$. We note that $L_{\text {sym }}^{2}\left(\left\{x:|x|=R_{0}\right\}\right)=H_{\text {sym }}^{0}\left(\left\{x:|x|=R_{0}\right\}\right.$. From the formulae for $u_{j}$ and $\Psi_{j}$ it follows immediately that

$$
\begin{aligned}
u_{0} & =(1-\alpha) /(1+\alpha)=1 / a_{0}, & & \\
u_{2 j} & =(-1)^{j} \cos 2 j \theta+O\left(R_{0}^{-2 j}\right) & & \text { on } \partial \Omega=\left\{x:|x|=R_{0}\right\}, \quad 1 \leqq j \\
u_{2 j+1} & =(-1)^{j} \sin (2 j+1) \theta+O\left(R_{0}^{-2 j-1}\right) & & \text { on } \partial \Omega=\left\{x:|x|=R_{0}\right\}, \quad 0 \leqq j .
\end{aligned}
$$

It is therefore not very surprising that we can prove the following density result.

Proposition 8.5. Given any $0 \leqq s$ there exists a constant $C_{s}<\infty$, so that $\operatorname{span}\left\{\left.u_{j}\right|_{\left\{x:|x|=R_{0}\right\}}\right\}$ is dense in $H_{\text {sym }}^{s}$, provided $R_{0}>C_{s}$.

Proof. Given $g \in L_{\text {sym }}^{2}\left(\left\{x:|x|=R_{0}\right\}\right)$, let $\left\{\gamma_{j}\right\}$ denote the coefficients of the Fourier expansion

$$
g=\gamma_{0} \frac{1}{a_{0}}+\sum_{j=1}^{\infty} \gamma_{2 j}(-1)^{j} \cos 2 j \theta+\sum_{j=0}^{\infty} \gamma_{2 j+1}(-1)^{j} \sin (2 j+1) \theta .
$$

We then have

$$
2\left(\frac{\gamma_{0}}{a_{0}}\right)^{2}+\sum_{j=1}^{\infty} \gamma_{j}^{2}=\frac{1}{R_{0} \pi} \int_{\left\{x:|x|=R_{0}\right\}} g^{2} d \sigma=\frac{1}{R_{0} \pi}\|g\|_{L^{2}\left(\left\{x:|x|=R_{0}\right\}\right)}^{2} .
$$


Now define $P g \in L_{\text {sym }}^{2}\left(\left\{x:|x|=R_{0}\right\}\right)$ by $P g=\left.\sum_{j=0}^{\infty} \gamma_{j} u_{j}\right|_{\left\{x:|x|=R_{0}\right\}}$.

Due to the definition of the $u_{j}$ we have, for $|x|=R_{0}>2$,

$$
g-P g=-\operatorname{Re} \sum_{j=1}^{\infty} \gamma_{j} R_{0}^{-j} \sum_{k=1}^{\infty}( \pm \alpha)^{k}\left[\frac{z^{j}}{(k z+\mathbf{i})^{j}} \mp \frac{z^{j}}{(k z-\mathbf{i})^{j}}\right]
$$

( + and - for $j$ odd, - and + for $j$ even). A simple calculation then gives

$$
\begin{aligned}
& \|g-P g\|_{L^{2}\left(\left\{x:|x|=R_{0}\right\}\right)} \leqq\left(\sum_{k=1}^{\infty}|\alpha|^{k}\right) \sum_{j=1}^{\infty}\left|\gamma_{j}\right| R_{0}^{-j}\left(\left\|\frac{z^{j}}{(z+\mathbf{i})^{j}}\right\|_{L^{2}\left(\left\{x:|x|=R_{0}\right\}\right)}\right. \\
& \left.+\left\|\frac{z^{j}}{(z-\mathbf{i})^{j}}\right\|_{L^{2}\left(\left\{x:|x|=R_{0}\right\}\right)}\right) \\
& \leqq\left(\sum_{k=1}^{\infty}|\alpha|^{k}\right) \sum_{j=1}^{\infty}\left|\gamma_{j}\right| R_{0}^{-j} \frac{2 R_{0}^{j}}{\left(R_{0}-1\right)^{j}} \sqrt{2 \pi R_{0}} \\
& \leqq C\left(\sum_{j=1}^{\infty}\left|\gamma_{j}\right|^{2}\right)^{1 / 2} \frac{1}{\sqrt{R_{0}}} \quad\left(R_{0}>2\right) \\
& \leqq \frac{C}{R_{0}}\|g\|_{L^{2}\left(\left\{x:|x|=R_{0}\right\}\right)} \\
& \leqq \frac{1}{2}\|g\|_{L^{2}\left(\left\{x:|x|=R_{0}\right\}\right)} \quad \text { (for } R_{0} \text { sufficiently large). }
\end{aligned}
$$

A similar calculation for the $H^{m}$ norm ( $m \geqq 1$, integer) yields

$$
\begin{aligned}
& \|g-P g\|_{H^{m}\left(\left\{x:|x|=R_{0}\right\}\right)} \\
& \leqq C\left(\sum_{k=1}^{\infty}|\alpha|^{k}\right) \sum_{l=0}^{m} \sum_{j=1}^{\infty}\left|\gamma_{j}\right| R_{0}^{-j}\left(\max _{1 \leqq k}\left\|\left(\frac{d}{d z}\right)^{l} \frac{z^{j}}{(k z+\mathbf{i})^{j}}\right\|_{L^{2}\left(\left\{x:|x|=R_{0}\right\}\right)}\right. \\
& \left.\quad+\max _{1 \leqq k}\left\|\left(\frac{d}{d z}\right)^{l} \frac{z^{j}}{(k z-\mathbf{i})^{j}}\right\|_{\left.L^{2}\left(\left\{x:|x|=R_{0}\right\}\right)\right)}\right) \\
& \leqq C\left(\sum_{k=1}^{\infty}|\alpha|^{k}\right) \sum_{l=0}^{m} \sum_{j=1}^{\infty}\left|\gamma_{j}\right| R_{0}^{-j}(j+l)^{l} \frac{R_{0}^{j}}{\left(R_{0}-1\right)^{j+l}} \sqrt{R_{0}} \\
& \left.\leqq C \sum_{l=0}^{m} \frac{1}{R_{0}^{l} \sqrt{R_{0}}} \int_{j=1}^{\infty}\left|\gamma_{j}\right|^{2} j^{2 l}\right)^{1 / 2} \quad\left(R_{0}>2\right) \\
& \leqq \frac{C}{R_{0}} \sum_{l=0}^{m}\left\|\left(\frac{\partial}{\partial \tau}\right)^{l} g\right\|_{L^{2}\left(\left\{x:|x|=R_{0}\right\}\right)} \\
& \leqq \frac{1}{2}\|g\|_{H^{m}\left(\left\{x:|x|=R_{0}\right\}\right) \quad\left(\text { for } R_{0} \text { sufficiently large }\right) .}
\end{aligned}
$$


Exactly how large $R_{0}$ has to be taken depends on the constant $C$, which depends on $m$. The two estimates (88) and (89), incidentally, also prove that $P g \in H_{s y m}^{m}$ if $g \in H_{s y m}^{m}, 0 \leqq m$. By interpolation we now immediately conclude that

$$
\|g-P g\|_{H^{s}\left(\left\{x:|x|=R_{0}\right\}\right)} \leqq \frac{1}{2}\|g\|_{H^{s}\left(\left\{x:|x|=R_{0}\right\}\right)} \quad \text { for } R_{0} \text { sufficiently large. }
$$

Exactly how large $R_{0}$ has to be depends on $s$, but not on $g$. Now let $r_{0}=g$, and define the sequence

$$
\begin{aligned}
& g_{1}=\operatorname{Pr}_{0} \quad r_{1}=r_{0}-g_{1} \\
& g_{2}=\operatorname{Pr}_{1} \quad r_{2}=r_{1}-g_{2} \\
& g_{k}=P r_{k-1} \quad r_{k}=r_{k-1}-g_{k}
\end{aligned}
$$

In other words

$$
g_{k}=P r_{k-1}, \quad r_{k}=g-\sum_{j=1}^{k} g_{j}, \quad \text { and } r_{k}=(I-P)^{k} g .
$$

From the estimate (90) we get

$$
\left\|r_{k}\right\|_{H^{s}\left(\left\{x:|x|=R_{0}\right\}\right)} \leqq\left(\frac{1}{2}\right)^{k}\|g\|_{H^{s}\left(\left\{x:|x|=R_{0}\right\}\right)},
$$

for $R_{0}$ sufficiently large. Equivalently

$$
\left\|g-\sum_{j=1}^{k} g_{j}\right\|_{H^{s}\left(\left\{x:|x|=R_{0}\right\}\right)} \leqq\left(\frac{1}{2}\right)^{k}\|g\|_{H^{s}\left(\left\{x:|x|=R_{0}\right\}\right)} .
$$

Since all the $g_{j}$ are elements of $\overline{\operatorname{span}}\left\{\left.u_{j}\right|_{\left\{x:|x|=R_{0}\right\}}\right\}$ (the closure refers to the $H^{s}$ topology) and since the latter forms a closed vectorspace, we have completed the proof of the proposition.

By a slight extension of the proof of the previous proposition we may prove the following, more detailed result.

Proposition 8.6. Suppose $0 \leqq s$ and suppose $R_{0}>C_{s}$. Any function $g \in H_{\text {sym }}^{s}$ ( $\left\{x:|x|=R_{0}\right\}$ ) may then be obtained as $g=\lim _{k \rightarrow \infty} h_{k}$, with

$$
h_{k}=\left.\sum_{j=0}^{\infty} \beta_{j}^{(k)} u_{j}\right|_{\left\{x:|x|=R_{0}\right\}} \in \overline{\operatorname{span}}\left\{\left.u_{j}\right|_{\left\{x:|x|=R_{0}\right\}}\right\},
$$

and

$$
\left(\sum_{j=0}^{\infty}\left|\beta_{j}^{(k)}\right|^{2}(j+1)^{2 s}\right)^{1 / 2} \leqq C\|g\|_{H^{s}}
$$

The limits and sums of functions all refer to the $H^{s}$ topology. The constant $C$ depends on $s$ and $R_{0}$, but is independent of $g$ and $k$. 
Proof. Let $r_{l}$ be as in the previous proof, and decompose it in terms of its Fourier Series

$$
r_{l}=\gamma_{0}^{(l)} \frac{1}{a_{0}}+\sum_{j=1}^{\infty} \gamma_{2 j}^{(l)}(-1)^{j} \cos 2 j \theta+\sum_{j=0}^{\infty} \gamma_{2 j+1}^{(l)}(-1)^{j} \sin (2 j+1) \theta
$$

Then

$$
\left(\sum_{j=0}^{\infty}\left|\gamma_{j}^{(l)}\right|^{2}(j+1)^{2 s}\right)^{1 / 2} \leqq C\left\|r_{l}\right\|_{H^{s}\left(\left\{x:|x|=R_{0}\right\}\right)},
$$

and thus according to (91)

$$
\left(\sum_{j=0}^{\infty}\left|\gamma_{j}^{(l)}\right|^{2}(j+1)^{2 s}\right)^{1 / 2} \leqq C\left(\frac{1}{2}\right)^{l}\|g\|_{H^{s}\left(\left\{x:|x|=R_{0}\right\}\right)}
$$

Also let $g_{l+1}=P r_{l}=\left.\sum_{j=0}^{\infty} \gamma_{j}^{(l)} u_{j}\right|_{\left\{x:|x|=R_{0}\right\}}$, as in the previous proof. The functions $h_{k}=\sum_{l=1}^{k} g_{l}$ converge to $g$ in $H^{s}\left(\left\{x:|x|=R_{0}\right\}\right)$, and they are elements of $\overline{\operatorname{span}}\left\{\left.u_{j}\right|_{\left\{x:|x|=R_{0}\right\}}\right\}$, with the representation

$$
h_{k}=\left.\sum_{j=0}^{\infty} \beta_{j}^{(k)} u_{j}\right|_{\left\{x:|x|=R_{0}\right\}}, \quad \beta_{j}^{(k)}=\sum_{l=0}^{k-1} \gamma_{j}^{(l)} .
$$

We now calculate

$$
\left(\sum_{j=0}^{\infty}\left|\beta_{j}^{(k)}\right|^{2}(j+1)^{2 s}\right)^{1 / 2} \leqq \sum_{l=0}^{k-1}\left(\sum_{j=0}^{\infty}\left|\gamma_{j}^{(l)}\right|^{2}(j+1)^{2 s}\right)^{1 / 2} \leqq C \sum_{l=0}^{k-1}\left(\frac{1}{2}\right)^{l}\|g\|_{H^{s}},
$$

so that

$$
\left(\sum_{j=0}^{\infty}\left|\beta_{j}^{(k)}\right|^{2}(j+1)^{2 s}\right)^{1 / 2} \leqq 2 C\|g\|_{H^{s}\left(\left\{x:|x|=R_{0}\right\}\right)},
$$

as desired.

We are now ready for

Proof of Proposition 8.1. We may without loss of generality suppose that $g$ is smooth on $\partial \Omega=\left\{x:|x|=R_{0}\right\}$. If not, simply choose $R_{0}^{\prime}$ and $R_{0}$, with $2<$ $R_{0}^{\prime}<R_{0}$, sufficiently large and so that $K \subset\left\{x:|x|=R_{0}^{\prime}\right\}$. By elliptic regularity $\left.u\right|_{\left\{x:|x|=R_{0}^{\prime}\right\}}$ is now smooth and we may proceed with $R_{0}$ replaced by $R_{0}^{\prime}$. Let $h_{k}$ be as guaranteed by Proposition 8.6 with some fixed $s>\frac{3}{2}$, i.e.,

$$
h_{k} \rightarrow g \text { in } H^{s}\left(\left\{x:|x|=R_{0}\right\}\right) \quad \text { and } h_{k}=\left.\sum_{j=0}^{\infty} \beta_{j}^{(k)} u_{j}\right|_{\left\{x:|x|=R_{0}\right\}}
$$


with

$$
\left(\sum_{j=0}^{\infty}\left|\beta_{j}^{(k)}\right|^{2}(j+1)^{2 s}\right)^{1 / 2} \leqq C\|g\|_{H^{s}} .
$$

It is not hard to see that

$$
\left|\frac{d}{d z} \Psi_{j}\right| \leqq C(j+1)
$$

in each of the three subdomains $\{z:|z+i| \leqq 1\},\{z:|z-i| \leqq 1\}$, and $\{z:|z| \leqq$ $\left.R_{0},|z+i| \geqq 1,|z-i| \geqq 1\right\}$, with the constant $C$ depending on $R_{0}$. Therefore

$$
\left\|u_{j}\right\|_{H^{1}(\Omega)} \leqq C(j+1),
$$

and consequently the infinite sums

$$
\tilde{U}_{k}=\sum_{j=0}^{\infty} \beta_{j}^{(k)} u_{j}
$$

are convergent in $H^{1}(\Omega)$, with

$$
\begin{aligned}
\left\|\tilde{U}_{k}\right\|_{H^{1}(\Omega)} & \leqq C \sum_{j=0}^{\infty}\left|\beta_{j}^{(k)}\right|(j+1) \\
& \leqq C\left(\sum_{j=0}^{\infty}\left|\beta_{j}^{(k)}\right|^{2}(j+1)^{2 s}\right)^{1 / 2}\left(\sum_{j=0}^{\infty}(j+1)^{2(1-s)}\right)^{1 / 2} .
\end{aligned}
$$

The trace of $\tilde{U}_{k}$ on $\partial \Omega=\left\{x:|x|=R_{0}\right\}$ is equal to $h_{k}$. From the construction of the $u_{j}$ it now follows that $\tilde{U}_{k}$ is the solution to

$$
\partial_{i}\left(a(x) \partial_{i} \tilde{U}_{k}\right)=0 \text { in } \Omega \text {, with } \tilde{U}_{k}=h_{k} \text { on } \partial \Omega .
$$

Since $h_{k} \rightarrow g$ in $H^{1 / 2}(\partial \Omega)$ we have that $\tilde{U}_{k} \rightarrow u$ in $H^{1}(\Omega)$. By selecting $N(k)$ sufficiently large we may obtain finite sums

$$
U_{k}=\sum_{j=0}^{N(k)} \beta_{j}^{(k)} u_{j}
$$

so that $U_{k} \rightarrow u$ in $H^{1}(\Omega)$. These $U_{k}$ still satisfy

$$
\partial_{i}\left(a(x) \partial_{i} U_{k}\right)=0 \quad \text { in } \Omega .
$$

From elliptic regularity theory we furthermore know that

$$
D^{\mathbf{m}} U_{k}(x) \rightarrow D^{\mathbf{m}} u(x)
$$


at any point inside $\Omega$, not on either of the two circles $\{x:|x-(0, \pm 1)|=1\}$, and for any multi-index $\mathbf{m}$. From Proposition 8.4 we have that

$$
\left|D^{\mathbf{m}} u_{j}(x)\right| \leqq C_{\mathbf{m}} R_{0}^{-j}(j+|\mathbf{m}|)^{|\mathbf{m}|}
$$

in each of the three regions

$$
\begin{gathered}
\left\{\left(x_{1}, x_{2}\right):\left|\left(x_{1}, x_{2}\right)\right| \leqq 1,\left|\left(x_{1}, x_{2}\right)-(0,-1)\right|>1,\left|\left(x_{1}, x_{2}\right)-(0,1)\right|>1\right\}, \\
\left\{\left(x_{1}, x_{2}\right):\left|\left(x_{1}, x_{2}\right)\right| \leqq 1,\left|\left(x_{1}, x_{2}\right)-(0,-1)\right|<1\right\}, \quad \text { and } \\
\left\{\left(x_{1}, x_{2}\right):\left|\left(x_{1}, x_{2}\right)\right| \leqq 1,\left|\left(x_{1}, x_{2}\right)-(0,1)\right|<1\right\}
\end{gathered}
$$

and thus

$$
\begin{aligned}
\left|D^{\mathbf{m}} U_{k}(x)\right| & \leqq \sum_{j=0}^{N(k)}\left|\beta_{j}^{(k)}\right|\left|D^{\mathbf{m}} u_{j}(x)\right| \\
& \leqq C_{\mathbf{m}} \sum_{j=0}^{N(k)}\left|\beta_{j}^{(k)}\right| R_{0}^{-j}(j+|\mathbf{m}|)^{|\mathbf{m}|} \\
& \leqq C_{\mathbf{m}}\left(\sum_{j=0}^{\infty}\left|\beta_{j}^{(k)}\right|^{2}\right)^{1 / 2}\left(\sum_{j=0}^{\infty} R_{0}^{-2 j}(j+|\mathbf{m}|)^{2|\mathbf{m}|}\right)^{1 / 2} \\
& \leqq C_{\mathbf{m}} \quad \text { for any multi-index } \mathbf{m},
\end{aligned}
$$

in each of the above three regions. From (92) and (93) it now follows immediately that $u$ has the desired smoothness properties for $|x| \leqq 1$. In particular, from the formulae for the $u_{j}$, we know that any of the derivatives, $D^{\mathbf{m}} u_{j}$, has the same limit at the origin, whether we approach through the left cusp or through the right cusp. Since $U_{k}$ is a finite linear combination of $u_{j}$ 's, all its derivatives have the same "continuity property". The boundedness of $D^{\mathbf{m}+\mathbf{e}_{i}} U_{k}, \mathbf{e}_{1}=(1,0), \mathbf{e}_{2}=(0,1)$, expressed in (93), now implies

$$
\left|D^{\mathbf{m}} U_{k}(x)-D^{\mathbf{m}} U_{k}(y)\right| \leqq C_{\mathbf{m}}|x-y|
$$

even when $x$ and $y$ lie in different cusps. Using the convergence statement (92) we conclude that

$$
\left|D^{\mathbf{m}} u(x)-D^{\mathbf{m}} u(y)\right| \leqq C_{\mathbf{m}}|x-y|
$$

even when $x$ and $y$ lie in different cusps. This verifies the "continuity property" of $D^{\mathbf{m}} u(x)$ referred to in Remark 8.1.

For $x \in K$, but outside $|x| \leqq 1$, the desired smoothness of $u$ follows immediately from classical elliptic regularity results. 


\section{Appendix: An auxiliary regularity result}

In this appendix we present a proof of a known result concerning the piecewise smoothness of solutions to divergence form second order elliptic equations with piecewise smooth coefficients. The idea of the proof, the use of a reflection to reduce the problem to an elliptic system with complementing boundary conditions, was suggested to us by L. Nirenberg.

Let $B_{1}$ denote the unit ball of $\mathbb{R}^{n}, n \geqq 1, B_{1}^{+}=\left\{x=\left(x_{1}, \cdots, x_{n}\right) \mid x \in\right.$ $\left.B_{1}, x_{n}>0\right\}, B_{1}^{-}=\left\{x=\left(x_{1}, \cdots, x_{n}\right) \mid x \in B_{1}, x_{n}<0\right\}$. Suppose

$\left(A_{i j}^{( \pm)}\right) \in C^{\infty}\left(\overline{B_{1}^{ \pm}}\right)$are symmetric, positive definite matrix functions,

$$
b_{i}^{( \pm)}, c_{i}^{( \pm)}, d^{( \pm)} \in C^{\infty}\left(\overline{B_{1}^{ \pm}}\right), \quad 1 \leqq i \leqq n
$$

and

$$
h^{( \pm)}, g_{i}^{( \pm)} \in C^{\infty}\left(\overline{B_{1}^{ \pm}}\right), \quad 1 \leqq i \leqq n
$$

We define $A_{i j}(x)=A_{i j}^{( \pm)}(x), b_{i}(x)=b_{i}^{( \pm)}(x), c(x)=c_{i}^{( \pm)}(x), d(x)=d^{( \pm)}(x)$, $h(x)=h^{( \pm)}(x)$, and $g_{i}(x)=g_{i}^{( \pm)}(x)$ for $x \in B_{1}^{ \pm}$.

Theorem 9.1. Assume (94), (95), and (96). If $u \in H^{1}\left(B_{1}\right)$ denotes a solution to

$$
\partial_{i}\left(A_{i j}(x) \partial_{j} u+b_{i}(x) u\right)+c_{i}(x) \partial_{i} u+d(x) u=h(x)+\partial_{i} g_{i}(x) \text { in } B_{1},
$$

and $u^{ \pm}(x)=u(x)$ for $x \in \overline{B_{1}^{ \pm}}$, then $u^{ \pm} \in C^{\infty}\left(\overline{B_{r}^{ \pm}}\right)$for every $0<r<1$.

Proof. A piecewise smooth solution $u$ of (97) satisfies the matching conditions

$$
u^{+}=u^{-}, \quad A_{i n}^{+} \partial_{i} u^{+}+b_{n}^{+} u^{+}-g_{n}^{+}=A_{i n}^{-} \partial_{i} u^{-}+b_{n}^{-} u^{-}-g_{n}^{-} \quad \text { at } x_{n}=0 .
$$

For $x=\left(x^{\prime}, x_{n}\right) \in B_{1}^{+}$, define

$$
\begin{gathered}
\hat{u}^{+}\left(x^{\prime}, x_{n}\right)=u^{-}\left(x^{\prime},-x_{n}\right), \\
\hat{A}_{i j}^{+}(x)= \begin{cases}A_{i j}^{-}\left(x^{\prime},-x_{n}\right) & i, j<n, \quad \text { or } i=j=n, \\
-A_{i j}^{-}\left(x^{\prime},-x_{n}\right) & i<n, j=n, \quad \text { or } j<n, i=n,\end{cases} \\
\hat{b}_{i}^{+}(x)=b_{i}^{-}\left(x^{\prime},-x_{n}\right), \quad i<n, \quad \hat{b}_{n}^{+}(x)=-b_{n}^{-}\left(x^{\prime},-x_{n}\right), \\
\hat{c}_{i}^{+}(x)=c_{i}^{-}\left(x^{\prime},-x_{n}\right), \quad i<n, \quad \hat{c}_{n}^{+}(x)=-c_{n}^{-}\left(x^{\prime},-x_{n}\right), \\
\hat{g}_{i}^{+}(x)=g_{i}^{-}\left(x^{\prime},-x_{n}\right), \quad i<n, \quad \hat{g}_{n}^{+}(x)=-g_{n}^{-}\left(x^{\prime},-x_{n}\right),
\end{gathered}
$$

and

$$
\hat{d}^{+}(x)=d^{-}\left(x^{\prime},-x_{n}\right), \quad \hat{h}^{+}(x)=h^{-}\left(x^{\prime},-x_{n}\right) .
$$


Then $\hat{u}^{+}$satisfies

$$
\begin{aligned}
\partial_{i}\left(\hat{A}_{i j}^{+}(x) \partial_{j} \hat{u}^{+}\right. & \left.+\hat{b}_{i}^{+}(x) \hat{u}^{+}\right)+\hat{c}_{i}^{+}(x) \partial_{i} \hat{u}^{+}+\hat{d}^{+}(x) \hat{u}^{+} \\
& =\hat{h}^{+}(x)+\partial_{i} \hat{g}_{i}^{+}(x) \text { in } B_{1}^{+} .
\end{aligned}
$$

The matching conditions take the form

$$
\begin{aligned}
u^{+}-\hat{u}^{+}=0, & A_{i n}^{+} \partial_{i} u^{+}+\hat{A}_{i n}^{+} \partial_{i} \hat{u}^{+}+b_{n}^{+} u^{+}+\hat{b}_{n}^{+} \hat{u}^{+}-g_{n}^{+}-\hat{g}_{n}^{+}=0 \\
& \text { at } x_{n}=0 .
\end{aligned}
$$

So $\left(u^{+}, \hat{u}^{+}\right)$satisfies a strongly elliptic system in divergence form in $B_{1}^{+},(97)$ and (98), with boundary conditions, (99). We will verify that the boundary conditions (99) are complementing boundary conditions for the elliptic system (see AGMON, Douglis \& NiRENBERG [1]). The desired piecewise smoothness then follows from classical elliptic theory (see for instance [1] and [10]).

Consider now the elliptic system

$$
\tilde{A}_{i j}^{(k)}(x) \partial_{i j} u^{(k)}+\tilde{b}_{i}^{(k)}(x) \partial_{i} u^{(k)}+\tilde{c}^{(k)}(x) u^{(k)}=\tilde{h}^{(k)}(x) \quad \text { in } B_{1}^{+}, k=1,2,
$$

together with the boundary conditions

$$
u^{(1)}-u^{(2)}=0, \quad \sum_{k=1}^{2} \tilde{A}_{i n}^{(k)} \partial_{i} u^{(k)}+\sum_{k=1}^{2} \tilde{e}^{(k)} u^{(k)}=\tilde{\psi} \quad \text { on } x_{n}=0,
$$

where $\left(\tilde{A}_{i j}^{(k)}\right), \tilde{b}_{i}^{(k)}, \tilde{c}^{(k)}, \tilde{h}^{(k)}$, and $\tilde{\psi}$ are smooth functions on $\overline{B_{1}^{+}}$, and $\left(\tilde{A}_{i j}^{(k)}(x)\right)$ is a symmetric, positive definite matrix for $k=1,2$, and $x \in \overline{B_{1}^{+}}$.

Our proof of Theorem 9.1 is complete if we verify that the boundary conditions (101) are complementing boundary conditions for the elliptic system (100). Let $\xi=\left(\xi^{\prime}, \tau\right)$ where $\xi^{\prime}=\left(\xi_{1}, \cdots, \xi_{n-1}\right) \neq 0$ denotes a real vector, while $\xi_{n} \equiv \tau$ runs in the field of complex numbers. Define

$$
\left(l_{i j}^{\prime}(x, \xi)\right)=\left(\begin{array}{cc}
\tilde{A}_{i j}^{(1)}(x) \xi_{i} \xi_{j} & 0 \\
0 & \tilde{A}_{i j}^{(2)}(x) \xi_{i} \xi_{j}
\end{array}\right)
$$

and

$$
L(x, \xi)=\operatorname{det}\left(l_{i j}^{\prime}(x, \xi)\right)=\left[A_{i j}^{(1)}(x) \xi_{i} \xi_{j}\right]\left[A_{i j}^{(2)}(x) \xi_{i} \xi_{j}\right] .
$$

The matrix $\left(L^{j m}(x, \xi)\right)$, consisting of the (transposed) cofactors of $\left(l_{i j}^{\prime}(x, \xi)\right)$, is given by

$$
\left(L^{j m}(x, \xi)\right)=\left(\begin{array}{cc}
\tilde{A}_{i j}^{(2)}(x) \xi_{i} \xi_{j} & 0 \\
0 & \tilde{A}_{i j}^{(1)}(x) \xi_{i} \xi_{j}
\end{array}\right)
$$

Define also

$$
\left(B_{h j}(x, \xi)\right)=\left(\begin{array}{cc}
1 & -1 \\
\tilde{A}_{i n}^{(1)}(x) \xi_{i} & \tilde{A}_{i n}^{(2)}(x) \xi_{i}
\end{array}\right)
$$


The product of the matrix $\left(B_{h j}(x, \xi)\right)$ and the matrix $\left(L^{j m}(x, \xi)\right)$, denoted $\left(D_{h m}(x, \xi)\right)$, has the form

$$
\left(D_{h m}(x, \xi)\right)=\left(\begin{array}{cc}
\tilde{A}_{i j}^{(2)}(x) \xi_{i} \xi_{j} & -\tilde{A}_{i j}^{(1)}(x) \xi_{i} \xi_{j} \\
{\left[\tilde{A}_{i n}^{(1)}(x) \xi_{i}\right]\left[\tilde{A}_{i j}^{(2)}(x) \xi_{i} \xi_{j}\right]} & {\left[\tilde{A}_{i n}^{(2)}(x) \xi_{i}\right]\left[\tilde{A}_{i j}^{(1)}(x) \xi_{i} \xi_{j}\right]}
\end{array}\right)
$$

We view $\tilde{A}_{i j}^{(1)}(x) \xi_{i} \xi_{j}$ as a polynomial in $\tau$ and factorize it as

$$
\tilde{A}_{i j}^{(1)}(x) \xi_{i} \xi_{j}=\tilde{A}_{n n}^{(1)}(x)\left[\tau-\tau_{1}^{+}\left(x, \xi^{\prime}\right)\right]\left[\tau-\tau_{1}^{-}\left(x, \xi^{\prime}\right)\right]
$$

where $\tau_{1}^{+}\left(\xi^{\prime}\right)$ denotes the root of $\tilde{A}_{i j}^{(1)}(x) \xi_{i} \xi_{j}=0$ with positive imaginary part, and $\tau_{1}^{-}\left(\xi^{\prime}\right)$ denotes its complex conjugate. Similarly, we write

$$
\tilde{A}_{i j}^{(2)}(x) \xi_{i} \xi_{j}=\tilde{A}_{n n}^{(2)}(x)\left[\tau-\tau_{2}^{+}\left(x, \xi^{\prime}\right)\right]\left[\tau-\tau_{2}^{-}\left(x, \xi^{\prime}\right)\right]
$$

Introduce

$$
M^{+}\left(x, \xi^{\prime}, \tau\right)=\left[\tau-\tau_{1}^{+}\left(\xi^{\prime}\right)\right]\left[\tau-\tau_{2}^{+}\left(\xi^{\prime}\right)\right],
$$

and regard $M^{+}\left(x, \xi^{\prime}, \tau\right)$ and the elements of the matrix $\left(D_{h m}(x, \xi)\right)$ as polynomials in $\tau$. Then, to verify that the boundary conditions are complementing, we only need to verify that, for fixed $x$ (on the boundary) the rows of the matrix $\left(D_{h m}(x, \xi)\right)$ are linearly independent modulo $M^{+}\left(x, \xi^{\prime}, \tau\right)$. In the following we drop the explicit reference to the fixed boundary point, $x=\left(x^{\prime}, 0\right)$, to simplify our notation.

For any fixed nonzero real vector $\xi^{\prime}$, let $C_{1}\left(\xi^{\prime}\right)$ and $C_{2}\left(\xi^{\prime}\right)$ be complex numbers such that

$$
\sum_{h=1}^{2} C_{h}\left(\xi^{\prime}\right) D_{h m}(\xi) \equiv 0\left(\bmod \mathrm{M}^{+}\left(\xi^{\prime}, \tau\right)\right), \quad \mathrm{m}=1,2
$$

We want to show that $C_{1}\left(\xi^{\prime}\right)=C_{2}\left(\xi^{\prime}\right)=0$. It follows from (102) that, for some complex numbers $\mu_{1}\left(\xi^{\prime}\right)$ and $\mu_{2}\left(\xi^{\prime}\right)$

$$
\begin{gathered}
C_{1}\left(\xi^{\prime}\right) \tilde{A}_{i j}^{(2)} \xi_{i} \xi_{j}+C_{2}\left(\xi^{\prime}\right)\left[\tilde{A}_{i n}^{(1)} \xi_{i}\right]\left[\tilde{A}_{i j}^{(2)} \xi_{i} \xi_{j}\right] \\
=C_{2}\left(\xi^{\prime}\right) \tilde{A}_{n n}^{(1)} \tilde{A}_{n n}^{(2)}\left(\tau-\mu_{1}\left(\xi^{\prime}\right)\right) M^{+}\left(\xi^{\prime}, \tau\right) \\
-C_{1}\left(\xi^{\prime}\right) \tilde{A}_{i j}^{(1)} \xi_{i} \xi_{j}+C_{2}\left(\xi^{\prime}\right)\left[\tilde{A}_{i n}^{(2)} \xi_{i}\right]\left[\tilde{A}_{i j}^{(1)} \xi_{i} \xi_{j}\right] \\
=C_{2}\left(\xi^{\prime}\right) \tilde{A}_{n n}^{(1)} \tilde{A}_{n n}^{(2)}\left(\tau-\mu_{2}\left(\xi^{\prime}\right)\right) M^{+}\left(\xi^{\prime}, \tau\right) .
\end{gathered}
$$


If $C_{1}\left(\xi^{\prime}\right)$ and $C_{2}\left(\xi^{\prime}\right)$ are not both zero, then the determinant of the coefficient matrix is identically zero, as a polynomial in $\tau$. That is

$$
\left[\sum_{k=1}^{2} \tilde{A}_{i n}^{(k)} \xi_{i}\right] L(\xi)-\tilde{A}_{n n}^{(1)} \tilde{A}_{n n}^{(2)} \sum_{k=1}^{2}\left(\tau-\mu_{k}\right)\left[\tilde{A}_{i j}^{(k)} \xi_{i} \xi_{j}\right] M^{+}\left(\xi^{\prime}, \tau\right) \equiv 0,
$$

and from this we deduce

$$
\left[\sum_{k=1}^{2} \tilde{A}_{i n}^{(k)} \xi_{i}\right]\left(\tau-\tau_{1}^{-}\right)\left(\tau-\tau_{2}^{-}\right)-\sum_{k=1}^{2} \tilde{A}_{n n}^{(k)}\left(\tau-\mu_{k}\right)\left(\tau-\tau_{k}^{-}\right)\left(\tau-\tau_{k}^{+}\right) \equiv 0 .
$$

It is easy to see from the above that

$$
\mu_{1}\left(\xi^{\prime}\right)=\tau_{2}^{-} \text {and } \mu_{2}\left(\xi^{\prime}\right)=\tau_{1}^{-} .
$$

Therefore,

$$
\sum_{k=1}^{2}\left[\tilde{A}_{i n}^{(k)} \xi_{i}-\tilde{A}_{n n}^{(k)}\left(\tau-\tau_{k}^{+}\right)\right] \equiv 0
$$

For $\tau=0$, the imaginary part of the left-hand side of this identity is clearly positive - a contradiction. We have verified that the boundary conditions are indeed complementing.

Note added after acceptance. It has been brought to our attention that a result very similar to Proposition 2.1 was established earlier by M. СHiPot, D. Kinderlehrer \& G.V. CAfFarelli. The reference is "Smoothness of linear laminates, Arch. Rational Mech. Anal. 96 (1986), pp. 81-96", and the particular result is Theorem 2. We note that the proof presented in that paper applies to elliptic systems, but is completely different from our proof of Proposition 2.1.

Acknowledgements. This work was partially supported by a Rutgers University Research Council grant (Y.-Y. LI), by NSF grant DMS-9706887 (Y.-Y. LI), and by NSF grant DMS9704575 (M. Vogelius). Part of this work was completed while the first author was visiting Département de Mathématiques, Université Paris VI, and while the second author was visiting the Mittag-Leffler Institute, Djursholm, Sweden. The authors thank their respective hosts, T. Aubin \& V. ThомéE, and these institutions for the kind invitations and hospitality.

\section{References}

1. S. Agmon, A. Douglis \& L. Nirenberg, Estimates near the boundary for solutions of elliptic partial differential equations satisfying general boundary conditions II. Comm. Pure Appl. Math. 17 (1964), pp. 35-92.

2. I. Babuska, B. Andersson, P.J. Smith \& K. Levin, Damage analysis of fiber composites. Part I: statistical analysis on fiber scale. The Aeronautical Research Institute of Sweden, Technical Note \# 15, 1998.

3. E. Bonnetier \& M. Vogelius, An elliptic regularity result for a composite medium with "touching" fibers of circular cross-section. SIAM J. Math. Anal. 31 (2000), pp. 651-677. 
4. B. Budiansky \& G.F. Carrier, High shear stresses in stiff fiber composites. J. App. Mech. 51 (1984), pp. 733-735.

5. L. Caffarelli, Interior a priori estimates for solutions of fully nonlinear equations. Ann. of Math. 130 (1989), 189-213.

6. L. Caffarelli \& X. Cabre, Fully Nonlinear Elliptic Equations, AMS Colloqium Publications 43, AMS, Providence, RI, 1995.

7. M. Giaginta, Multiple integrals in the calculus of variations and nonlinear elliptic systems, Ann. Math. Studies 105, Princeton University, N.J. 1983.

8. D. Gilbarg \& N.S. Trudinger, Elliptic Partial Differential Equations of Second Order. Springer-Verlag, 1983.

9. X. MARKENSCOFF, Stress amplification in vanishingly small geometries. Computational Mechanics 19 (1996), 77-83.

10. M. Schechter, A generalization of the problem of transmission. Ann. Scuola Norm. Sup. Pisa 4 (1960), 207-236.

\author{
Department of Mathematics \\ Rutgers University \\ New Brunswick \\ NJ 08903, USA \\ e-mail: yyli@math.rutgers.edu; \\ vogelius@math.rutgers.edu
}

(Accepted June 8, 1999) 